\title{
New Logarithmic Operational Laws-Based Complex q-Rung Orthopair Fuzzy Aggregation Operators and Their Application in Decision-Making Process
}

\author{
Harish Garg (iD, ${ }^{1}$ Zeeshan Ali,, ${ }^{2}$ Tahir Mahmood ${ }^{D},{ }^{2}$ Sultan Aljahdali, ${ }^{3}$ and Habib Shah ${ }^{4}$ \\ ${ }^{1}$ School of Mathematics, Thapar Institute of Engineering and Technology (Deemed University), Patiala 147004, Punjab, India \\ ${ }^{2}$ Department of Mathematics and Statistics, International Islamic University Islamabad, Islamabad, Pakistan \\ ${ }^{3}$ Department of Computer Science, College of Computers and Information Technology, Taif University, P.O. Box 11099, \\ Taif 21944, Saudi Arabia \\ ${ }^{4}$ College of Computer Science, King Khalid University, Abha 62529, Saudi Arabia
}

Correspondence should be addressed to Harish Garg; harishg58iitr@gmail.com

Received 2 February 2021; Revised 9 June 2021; Accepted 20 July 2021; Published 26 August 2021

Academic Editor: Zhen Zhang

Copyright (C) 2021 Harish Garg et al. This is an open access article distributed under the Creative Commons Attribution License, which permits unrestricted use, distribution, and reproduction in any medium, provided the original work is properly cited.

\begin{abstract}
A complex q-rung orthopair fuzzy set (CQROFS) is one of the useful tools to handle the uncertainties in the data. The main characteristic of the CQROFS is that it handles the imprecise information in the data using the membership degrees such that the sum of the q-powers of the real parts (also for imaginary parts) of the membership and nonmembership degrees is restricted to the unit interval. Keeping the highlights of this set, in this study, we manifested some new logarithmic operational laws (LOLs) between the pairs of the CQROFSs and investigated their properties. Also, by using these proposed laws, we stated several averaging and geometric operators, namely, logarithmic complex q-rung orthopair fuzzy weighted averaging (LCQROFWA) and logarithmic complex q-rung orthopair fuzzy weighted geometric (LCQROFWG) operators and hence stated their fundamental properties. Later on, based on the suggested operators, a multiattribute decision-making (MADM) algorithm is acted to solve the decision-making problems. A numerical example has been considered to illustrate the approach and compare their obtained results with several of the existing studies' results. Finally, the advantages of the suggested algorithms and operators are presented.
\end{abstract}

\section{Introduction}

Decision-making procedure is a capable tool to handle awkward and intricate information in daily life issues. In the last few years, numerous scholars have implemented the decision-making technique in the environment of separated areas [1]. In the genuine decision-making process, a significant issue is a way to show the quality worth even more effectively and precisely. Because of the multifaceted nature of decision-making issues and the fuzziness of decisionmaking conditions, it is not enough to show attribute objects of alternatives by crisp number. To address this, the theory of intuitionistic fuzzy set (IFS) was investigated by Atanassove [2], by including the degree of falsity in the idea of the fuzzy set (FS) which was developed by Zadeh [3]. The rule of IFS is that the sum of both degrees cannot be exceeded from the unit interval, has extensive effectiveness, and is superior to FS to solve the problems. Several scholars have implemented the theory of IFS in numerous areas [4-8]. Later on, the theory of Pythagorean fuzzy set (PFS) was investigated by Yager [9], by using the rule that the sum of the square of both degrees cannot be exceeded by one. Several scholars have implemented the theory of PFS in numerous areas [10-15]. In real-life problems, there may occur some situations where the conditions of PFS are not satisfied. To address such problems, the theory of q-rung orthopair fuzzy set (QROFS) was investigated by Yager [16], with the rule that the sum of the q-power of both degrees is less than one. Several scholars have implemented the theory of QROFS in numerous areas [17-20]. 
Complex IFS (CIFS) was investigated by Alkouri and Salleh [21], by including the degree of falsity in the idea of complex FS (CFS) which was developed by Ramot [22]. CIFS contains the degree of truth and falsity in the form of a complex number with a rule that the sum of the real part (also for an imaginary part) of both degrees cannot be exceeded by one, has extensive effectiveness, and is superior to CFS to deal with those issues which cannot be resolved by using CFS theory. Several scholars have implemented the theory of CIFS in numerous areas [23-27]. Recently, the theory of complex PFS (CPFS) was investigated by Ullah et al. [28] under the assumption that the sum of the square of the real part (also for an imaginary part) of both degrees is less than one. This CPFS has extensive effectiveness and is superior to deal with the problem over the CIFS. Several scholars have implemented the theory of CPFS in numerous areas [29,30]. From the studies, it is observed that the scope of the CPFS is limited under the domain of all those degrees which satisfies the condition that the sum of the squares of the real part (also for an imaginary part) of both degrees cannot be exceeded by one. To get wider scope and range, a concept of complex QROFS (CQROFS) was investigated by Liu et al. [31] with the rule that the sum of the q-power of the real part (also for an imaginary part) of both degrees is less than one. Recently, Garg et al. [32] defined an algorithm for solving the decision-making problems under the complex interval-valued q-rung orthopair uncertain linguistic information.

The above-listed literature is mostly focused on the aggregation operator between the pairs of the different sets and they are linear. However, the existing operation laws and their operators have restricted access and they do not consider the nonlinear nature of the membership functions. With the growing complexity of the decision process in these days, the most challenging question that arises here is how to access the information in a reliable manner from the expert? The second question is how to aggregate the collective information of the expert? To answer the first question, the concept of CQROFS is a useful tool to handle the uncertainties in the data. The main characteristic of the CQROFS is that it handles the imprecise information in the data using the membership degrees such that the sum of the q-powers of the real parts (also for imaginary parts) of the membership and nonmembership degrees is restricted to the unit interval in polar coordinates. The amplitude term corresponding to truth and falsity degrees gives the extent of membership and nonmembership of an object in a CQROFS with a rule that the sum of the q-powers of both the grades is restricted to the unit interval. The phase terms are novel parameters of the truth and falsity degrees and these are the parameters that distinguish the CQROFS and traditional QROFS theories. To illustrate the significance of the phase term, we give an example. Assume XYZ organization chooses to set up biometric-based participation gadgets (BBPGs) in the entirety of its workplaces spread everywhere in the country. For this, the organization counsels a specialist who gives the data concerning (i) demonstrations of BBPGs and (ii) creation dates of BBPGs. The organization needs to choose the most ideal model of BBPGs with its creation date all the while. Here, the issue is two-dimensional, to be specific, the model of BBPGs and the creation date of BBPGs. This kind of issue cannot be displayed precisely utilizing the conventional QROFS hypothesis as the QROFS hypothesis cannot handle both measurements at the same time. The most ideal approach to address the entirety of the data given by the master is by utilizing the CQROFS hypothesis. The sufficiency terms in CQROFS might be utilized to give the organization's choice regarding the model of BBPGs and the stage terms might be utilized to address the organization's judgment concerning the creation date of BBPGs.

Next, to address the question of how to aggregate the collective information of the expert, for this, there is always a need to define some more nonlinear and generalized operation laws to perform the aggregation process without any loss of information. By considering the complexity of the decision-making process, either exponential or logarithm operational laws are the best-suited task for aggregation since the exponential and logarithm are inverse to each other. Furthermore, the features of the logarithm function, such as (i) its rate of change which can be represented graphically, (ii) the data value which can be elevated for small inputs, and (iii) representing exponential functions into linear functions, help the decision-maker to understand the information in a more precise manner. Also, the exponential functions are hard to understand, for the general people, thus, using the logarithmic application, simplification can be conducted. In this context, logarithm considers 10 as the base value of the function, and thereby large values can be plotted in the graph papers quite easily. Keeping the advantages of the logarithm function, Garg [14] firstly utilized the principle of logarithm operational laws (LOLs) in the environment of interval-valued Pythagorean fuzzy set and hence presented the LOL-based operator to aggregate the different information during the decision-making process. Also, in [14], the authors have introduced more generalized LOLs in which bases are either crisp or intervalvalued. Since their considered work is restricted under the domain of the Pythagorean fuzzy set, there is a need to extend their work to a more generalized CQROFS environment. For this, in this manuscript, we have presented a more generalized base of the logarithmic into $\rho_{C Q}$ and presented the concept of LOL for the CQROFS. By keeping the advantages of CQROFS which contain the degree of truth and falsity in the form of a complex number belonging to unit disc in a complex plane, the summary of the investigated approaches is discussed as follows:

(1) To explore the LOLs and their properties.

(2) To investigate the weighted averaging and geometric operators based on logarithmic complex q-rung orthopair fuzzy sets. Some special cases of the presented operators are also discussed in detail.

(3) A multiattribute decision-making (MADM) technique is developed based on the proposed operators and illustrates it with some numerical examples.

(4) The advantages, comparative analysis, and graphical expressions of the discovered theory are also discussed. 
The rest of the work is organized as follows. in Section 2, we recall the idea of CQROFSs and their laws. In Section 3, we developed the theory of LOLs based on CQROFS and its properties. In Section 4, some new weighted averaging and geometric operators based on the LOLs are defined and their fundamental properties are investigated. In Section 5, an algorithm for the MADM approach has been presented based on the proposed operators and illustrated with some numerical examples. Finally, to improve the quality of the explored approaches, comparative analysis, advantages, and graphical representations are also demonstrated. The conclusion of this manuscript is discussed in Section 6.

\section{Preliminaries}

The purpose of this section is to recall some fundamental laws such as CQROFSs and their operational laws.

Definition 1 (see [31]). A CQROFS $\mathfrak{夭}_{C Q}$ is demonstrated by

$$
\mathfrak{c}_{C Q}=\left\{\left(\mathfrak{M}_{\mathfrak{E}_{C Q}}(\widetilde{x}), \mathfrak{N}_{\mathfrak{E}_{C Q}}(\widetilde{x})\right): \widetilde{x} \in \widetilde{\mathscr{X}}\right\},
$$

where $\quad \mathfrak{M}_{\mathfrak{E}_{C Q}}(\widetilde{x})=\mathfrak{M}_{\mathfrak{C}_{R P}}(\overbrace{x}^{x}) e^{i 2 \pi\left(\mathfrak{M}_{\mathfrak{E}_{I P}}(\overbrace{x}^{x})\right)} \quad$ and

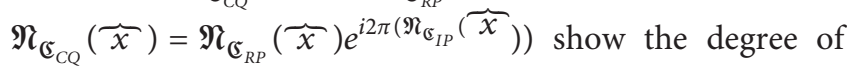
agreement and the degree of disagreeing with

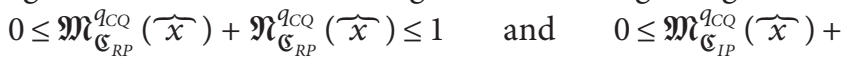
$\mathfrak{N}_{\mathfrak{E}_{I P}}^{q_{C Q}}(\overbrace{x}) \leq 1, q_{C Q} \geq 1$. Moreover, the term

$$
\begin{aligned}
& \mathscr{I}_{\mathbb{E}_{C Q}}(\widetilde{x})=\mathscr{I}_{\mathbb{C}_{R P}}(\widetilde{x}) e^{i 2 \pi\left(\mathscr{I}_{\mathbb{E}_{I P}}\right)}
\end{aligned}
$$

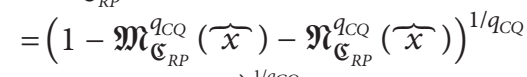

$$
\begin{aligned}
& e^{i 2 \pi\left(1-\mathfrak{M}_{\mathfrak{E}_{I P}}^{q C Q}(\overbrace{}^{x})-\mathfrak{N}_{\mathfrak{E}_{I P}}^{q C Q}(\overbrace{}^{x})\right)^{1 / q C Q}}
\end{aligned}
$$

expresses the degree of indeterminacy. Throughout this manuscript, the complex q-rung orthopair fuzzy numbers (CQROFNs) are shown by $\mathfrak{C}_{C Q}=\left(\mathfrak{M}_{\mathfrak{C}_{R P}} e^{i 2 \pi\left(\mathfrak{M}_{\mathbb{E}_{I P}}\right)}, \mathfrak{N}_{\mathfrak{C}_{R P}} e^{i 2 \pi\left(\mathfrak{N}_{\mathbb{E}_{I P}}\right)}\right)$. Additionally, by using $\mathfrak{S}_{C Q}=\left(\mathfrak{M}_{\mathfrak{C}_{R P}} e^{i 2 \pi\left(\mathfrak{M}_{\mathfrak{C}_{I P}}\right)}, \mathfrak{N}_{\mathfrak{C}_{R P}} e^{i 2 \pi\left(\mathfrak{N}_{\mathfrak{C}_{I P}}\right)}\right)$, we define the score and accuracy values such that

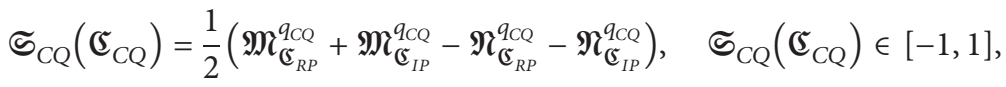

$$
\begin{aligned}
& \mathfrak{H}_{C Q}\left(\mathfrak{夭}_{C Q}\right)=\frac{1}{2}\left(\mathfrak{M}_{\mathfrak{C}_{R P}}^{q_{C Q}}+\mathfrak{M}_{\mathfrak{C}_{I P}}^{q_{C Q}}+\mathfrak{N}_{\mathfrak{C}_{R P}}^{q_{C Q}}+\mathfrak{N}_{\mathfrak{C}_{I P}}^{q_{C Q}}\right), \quad \mathfrak{H}_{C Q}\left(\mathfrak{c}_{C Q}\right) \in[0,1] .
\end{aligned}
$$

To find the relationships between any number two CQROFNs $\mathfrak{c}_{C Q-1}$ and $\mathfrak{c}_{C Q-2}$, we use the following rules:

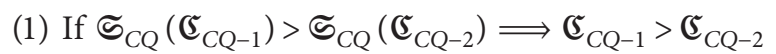

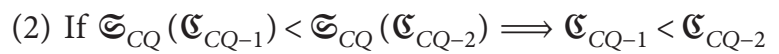

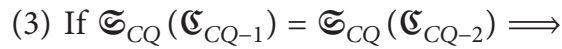

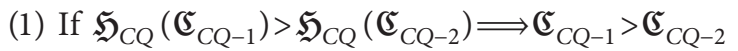

(2) If $\mathfrak{S}_{C Q}\left(\mathfrak{c}_{C Q-1}\right)<\mathfrak{H}_{C Q}\left(\mathfrak{⿰}_{C Q-2}\right) \Longrightarrow \mathfrak{c}_{C Q-1}<\mathfrak{c}_{C Q-2}$

Definition 2 (see [31]). Based on $\mathfrak{c}_{C Q-1}=\left(\mathfrak{M}_{\mathfrak{C}_{R P-1}}\right.$ $\left.e^{i 2 \pi\left(\mathfrak{M}_{\mathfrak{C}_{I P-1}}\right)}, \mathfrak{N}_{\mathfrak{C}_{R P-1}} e^{i 2 \pi\left(\mathfrak{N}_{\mathfrak{C}_{I P-1}}\right)}\right) \quad$ and $\quad \mathfrak{C}_{C Q-2}=\left(\mathfrak{M}_{\mathfrak{C}_{R P-2}}\right.$ $\left.e^{i 2 \pi\left(\mathfrak{M}_{\mathbb{C}_{I P-2}}\right)}, \mathfrak{N}_{\mathfrak{C}_{R P-2}} e^{i 2 \pi\left(\mathfrak{N}_{\mathbb{C}_{I P-2}}\right)}\right), k>0$ is a real number; then, we discuss the following laws:

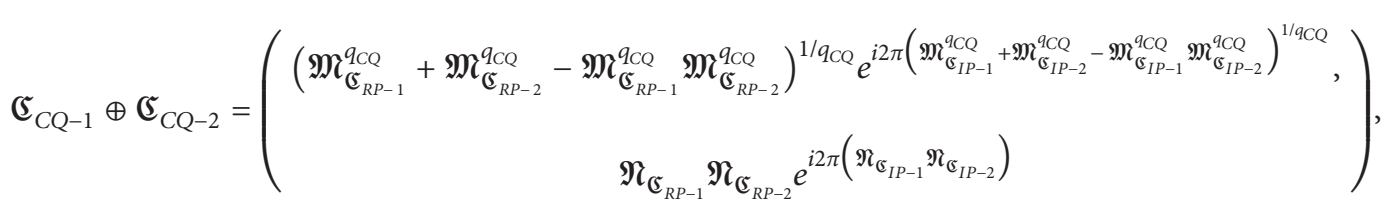

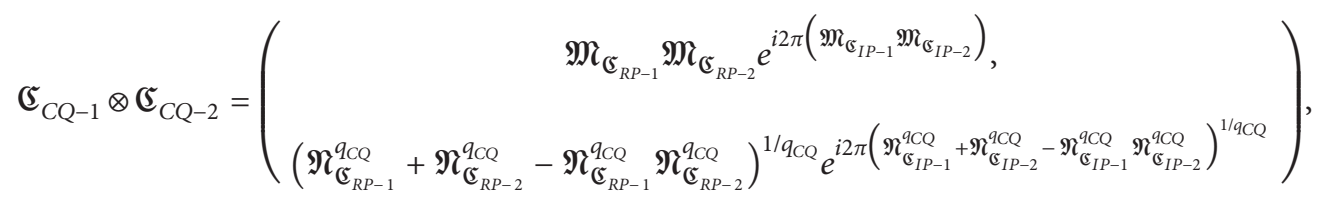

$$
\begin{aligned}
& k \mathfrak{S}_{C Q-1}=\left(\left(1-\left(1-\mathfrak{M}_{\mathfrak{C}_{R P-1}}^{q_{C Q}}\right)^{k}\right)^{1 / q_{C Q}} e^{i 2 \pi\left(1-\left(1-\mathfrak{M}_{\mathfrak{E}_{I P-1}}^{q_{C Q}}\right)^{k}\right)^{1 / q C Q}}, \mathfrak{N}_{\mathfrak{C}_{R P-1}}^{k} e^{i 2 \pi\left(\mathfrak{N}_{\mathfrak{G}_{I P-1}}^{k}\right)}\right),
\end{aligned}
$$




$$
\begin{aligned}
& \mathfrak{夭}_{C Q-1}^{k}=\left(\mathfrak{M}_{\mathfrak{夭}_{R P-1}}^{k} e^{i 2 \pi\left(\mathfrak{M}_{\mathfrak{G}_{I P-1}}^{k}\right)},\left(1-\left(1-\mathfrak{N}_{\mathfrak{夭}_{R P-1}}^{q_{C Q}}\right)^{k}\right)^{1 / q_{C Q}} e^{i 2 \pi\left(1-\left(1-\mathfrak{N}_{\mathfrak{G}_{I P-1}}^{q_{C Q}}\right)^{k}\right)^{1 / q_{C Q}}}\right),
\end{aligned}
$$

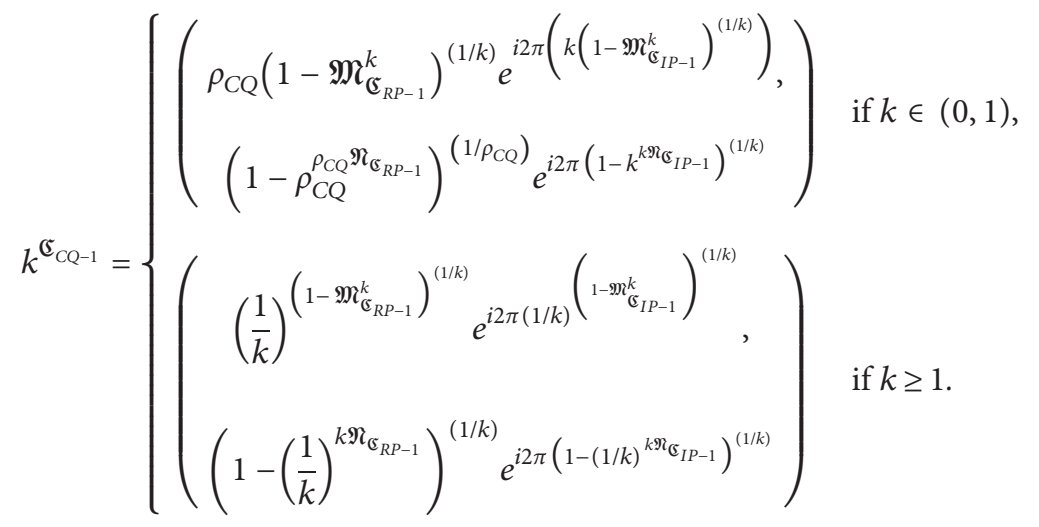

Liu et al. [31] discovered the theory of aggregation operators based on CQROFSs, which are discussed below.
Definition 3 (see [31]). For any family of CQROFNs $\mathfrak{S}_{C Q-\ell}=\left(\mathfrak{M}_{\mathfrak{C}_{R P-\ell}} e^{i 2 \pi\left(\mathfrak{M}_{\mathfrak{C}_{I P-\ell}}\right)}, \mathfrak{N}_{\mathfrak{C}_{R P-\ell}} e^{i 2 \pi\left(\mathfrak{N}_{\mathfrak{C}_{I P-\ell}}\right)}\right), \quad \ell=1,2, \ldots$, $\tilde{n}$, the complex q-rung orthopair fuzzy weighted averaging (CQROFWA) operators is demonstrated by

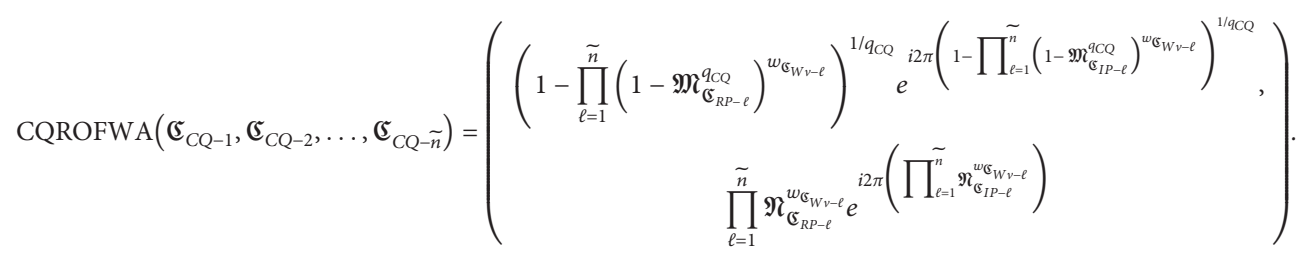

And the complex q-rung orthopair fuzzy ordered weighted averaging (CQROFOWA) operators is demonstrated by

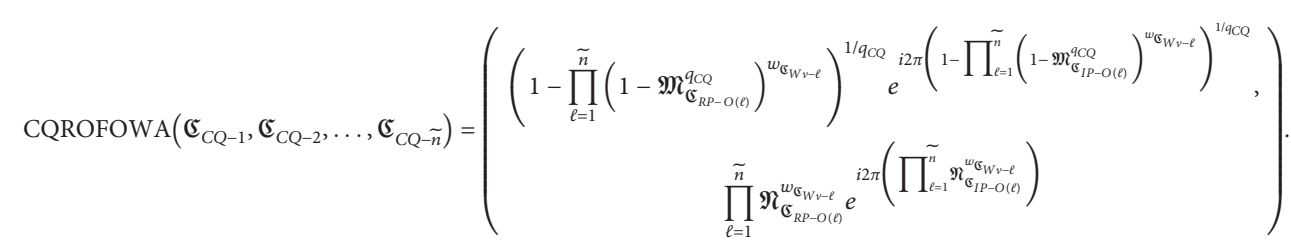

\section{Logarithmic Operational Laws and Their Based Aggregation Operators of CQROFSs}

The purpose of this study is to present new operations laws named as logarithm operations laws (LOLs) for the pairs of the CQROFNs. Based on these laws, we explore the ideas of aggregation operators for CQROFNs. Suppose $\boldsymbol{}_{C Q}$ and $\rho_{C Q}$ express the CQROFN and real number, where $\log _{\rho_{C Q}} 0$ and $\log _{1} \overbrace{x}$ is not investigated in positive numbers. We suppose that CQROFN $\mathfrak{c}_{C Q}=\left(\mathfrak{M}_{\mathfrak{C}_{R P}} e^{i 2 \pi\left(\mathfrak{M}_{\left.\mathbb{C}_{I P}\right)}\right)}, \mathfrak{N}_{\mathfrak{C}_{R P}} e^{i 2 \pi\left(\mathfrak{N}_{\mathfrak{C}_{I P}}\right)}\right) \neq 0=$ $\left(0 e^{i 2 \pi(0)}, 0 e^{i 2 \pi(0)}\right)$ and $\rho_{C Q} \neq 1$ in all manuscripts.

Definition 4 For any family of CQROFNs $\mathfrak{S}_{C Q}=\left(\mathfrak{M}_{\mathfrak{C}_{R P}} e^{i 2 \pi\left(\mathfrak{M}_{\mathbb{C}_{I P}}\right)}, \mathfrak{N}_{\mathfrak{C}_{R P}} e^{i 2 \pi\left(\mathfrak{N}_{\mathbb{E}_{I P}}\right)}\right)$, then the LOL for CQROFN is demonstrated by 


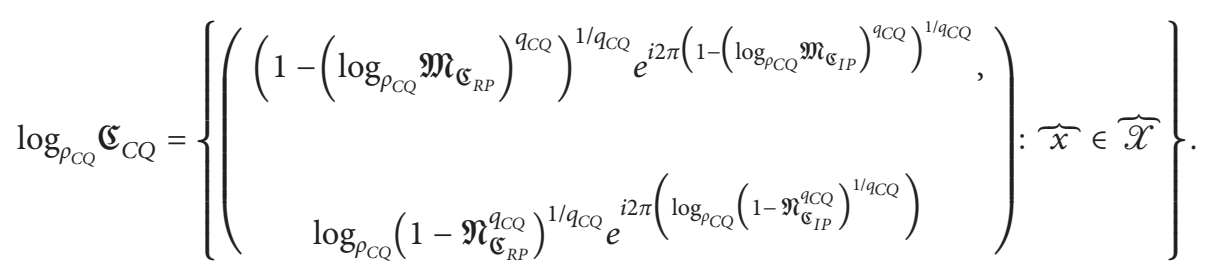

From equation (8), it is clear that it is CQROFS with rules such that $0 \leq \mathfrak{M}_{\mathfrak{C}_{R P}}^{q_{C Q}}+\mathfrak{N}_{\mathfrak{C}_{R P}}^{q_{C Q}} \leq 1$ and $0 \leq \mathfrak{M}_{\mathfrak{C}_{I P}}^{q_{C Q}}+\mathfrak{N}_{\mathfrak{C}_{I P}}^{q_{C Q}} \leq 1, q_{C Q} \geq 1$. For the real part of truth degree, $\quad$ if $\quad 0<\rho_{C Q} \leq \min \left\{\mathfrak{M}_{\mathfrak{C}_{R P}},\left(1-\mathfrak{N}_{\mathfrak{C}_{R P}}^{q_{C Q}}\right)^{1 / q_{C Q}}\right\} \leq 1$ and $\rho_{C Q} \neq 1$ and also for imaginary part, if $0<\rho_{C Q} \leq \min \left\{\mathfrak{M}_{\mathfrak{E}_{I P}},\left(1-\mathfrak{N}_{\mathfrak{E}_{I P}}^{q_{C Q}}\right)^{1 / q_{C Q}}\right\} \leq 1$ and $\rho_{C Q} \neq 1$; then; equation (8) is a CQROFS.
Definition 5. For any family of CQROFNs $\mathfrak{S}_{C Q}=\left(\mathfrak{M}_{\mathfrak{C}_{R P}} e^{i 2 \pi\left(\mathfrak{M}_{\mathfrak{C}_{I P}}\right)}, \mathfrak{N}_{\mathfrak{C}_{R P}} e^{i 2 \pi\left(\mathfrak{N}_{\mathfrak{C}_{I P}}\right)}\right)$, the logarithmic complex q-rung orthopair fuzzy (LCQROF) operator is demonstrated by

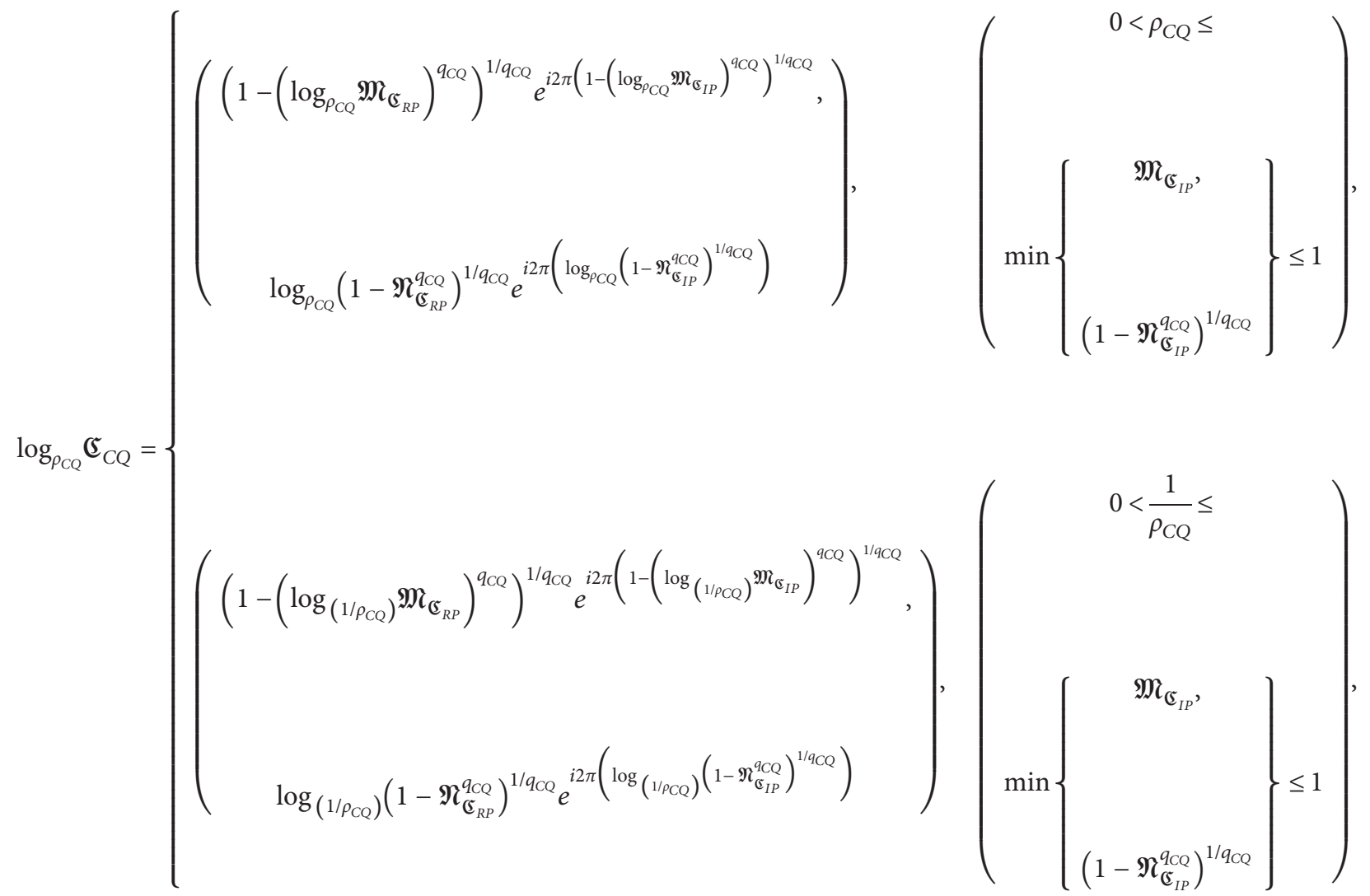

where $\log _{\rho_{C Q}} 0=0$ and $\rho_{C Q} \neq 1$.

Theorem 1. For any family of CQROFNs $\mathfrak{E}_{\mathrm{CQ}}=$ $\left(\mathfrak{M}_{\mathfrak{C}_{R P}} e^{i 2 \pi\left(\mathfrak{M}_{\mathfrak{C}_{I P}}\right)}, \mathfrak{N}_{\mathfrak{C}_{R P}} e^{i 2 \pi\left(\mathfrak{\Re}_{\mathfrak{C}_{I P}}\right)}\right)$, the $\log _{\rho_{C Q}} \mathfrak{S}_{C Q}$ is also CQROFN.
Proof. The CQROFNs $\quad \mathfrak{c}_{\mathrm{CQ}}=\left(\mathfrak{M}_{\mathfrak{E}_{R P}} e^{i 2 \pi\left(\mathfrak{M}_{\mathfrak{E}_{I P}}\right)}\right.$, $\left.\mathfrak{N}_{\mathfrak{C}_{R P}} e^{i 2 \pi\left(\mathfrak{\Re}_{\mathfrak{C}_{I P}}\right)}\right)$ with rules such that $0 \leq \mathfrak{M}_{\mathfrak{C}_{R P}}^{q_{C Q}}+\mathfrak{N}_{\mathfrak{C}_{R P}}^{q_{C Q}} \leq 1$ and $0 \leq \mathfrak{M}_{\mathfrak{G}_{I P}}^{q_{C Q}}+\mathfrak{N}_{\mathfrak{C}_{I P}}^{q_{C Q}} \leq 1, q_{C Q} \geq 1$. Then, we discuss the following two rules: 
(1) For the real part of the truth degree, if $0<\rho_{C Q} \leq \min \left\{\mathfrak{M}_{\mathfrak{C}_{R P}},\left(1-\mathfrak{N}_{\mathfrak{C}_{R P}}^{q_{C Q}}\right)^{1 / q_{C Q}}\right\} \leq 1 \quad$ and $\rho_{C Q} \neq 1$, then $\log _{\rho_{C Q}} \mathfrak{S}_{C Q}$ is decreasing based on $\rho_{C Q}$; therefore,

$0 \leq \log _{\rho_{C Q}} \mathfrak{M}_{\mathfrak{C}_{R P}}, \log _{\rho_{C Q}}\left(1-\mathfrak{N}_{\mathfrak{C}_{R P}}^{q_{C Q}}\right)^{1 / q_{C Q}} \leq 1 ; \quad$ then, $0 \leq\left(1-\left(\log _{\rho_{C Q}} \mathfrak{M}_{\mathfrak{C}_{R P}}\right)^{q_{C Q}}\right)^{1 / q_{C Q}} \leq 1 \quad$ and $0 \leq \log _{\rho_{C Q}}$ $\left(1-\mathfrak{N}_{\mathfrak{C}_{R P}}^{q_{C Q}}\right)^{1 / q_{C Q}} \leq 1$ and $0 \leq 1-\left(\log _{\rho_{C Q}} \mathfrak{M}_{\mathfrak{C}_{R P}}\right)^{q_{C Q}}+$ $\left(\log _{\rho_{C Q}}\left(1-\mathfrak{N}_{\mathfrak{C}_{R P}}^{q_{C Q}}\right)^{1 / q_{C Q}}\right)^{q_{C Q}} \leq 1$. For the imaginary part of truth degree, if $0<\rho_{C Q}$ $\leq \min \left\{\mathfrak{M}_{\mathfrak{C}_{I P}},\left(1-\mathfrak{N}_{\mathfrak{夭}_{I P}}^{q_{C Q}}\right)^{\left(1 / q_{C Q}\right)}\right\} \leq 1$ and $\rho_{C Q} \neq 1$, then $\log _{\rho_{C Q}} \mathfrak{E}_{C Q}$ is decreasing based on $\rho_{C Q}$; therefore, $0 \leq \log _{\rho_{C Q}} \mathfrak{M}_{\mathfrak{C}_{I P}}, \log _{\rho_{C Q}}\left(1-\mathfrak{N}_{\mathfrak{C}_{I P}}^{q_{C Q}}\right)^{1 / q_{C Q}} \leq 1 ; \quad$ then, $0 \leq\left(1-\left(\log _{\rho_{C Q}} \mathfrak{M}_{\mathfrak{E}_{I P}}\right)^{q_{C Q}}\right)^{1 / q_{C Q}} \leq 1$ and $0 \leq \log _{\rho_{C Q}}$ $\left(1-\mathfrak{N}_{\mathfrak{C}_{I P}}^{q_{C Q}}\right)^{1 / q_{C Q}} \leq 1 \quad$ and $\quad 0 \leq 1-\left(\log _{\rho_{C Q}} \mathfrak{M}_{\mathfrak{C}_{I P}}\right)^{q_{C Q}}$ $+\left(\log _{\rho_{C Q}}\left(1-\mathfrak{N}_{\mathfrak{C}_{I P}}^{q_{C Q}}\right)^{1 / q_{C Q}}\right)^{q_{C Q}} \leq 1$. Hence, $\log _{\rho_{C Q}} \mathfrak{夭}_{C Q}$ is CQROFN.

(2) If $\rho_{C Q}>1$ and $0<\left(1 / \rho_{C Q}\right)<1 \quad$ and $\left(1 / \rho_{C Q}\right) \leq \min \left\{\mathfrak{M}_{\mathfrak{C}_{R P},},\left(1-\mathfrak{N}_{\mathfrak{C}_{R P}}^{q_{C Q}}\right)^{1 / q_{C Q}}\right\} \leq 1 \quad$ and $\left(1 / \rho_{C Q}\right) \leq \min \left\{\mathfrak{M}_{\mathfrak{C}_{I P}},\left(1-\mathfrak{N}_{\mathfrak{C}_{I P}}^{q_{C Q}}\right)^{1 / q_{C Q}}\right\} \leq 1$, therefore $\log _{\rho_{C Q}} \mathfrak{r}_{C Q}$ is CQROFN.

We discussed the above two cases with the help of numerical example which is discussed below.

By using the value of $\rho_{C Q} \in(0,1)$, we investigate some properties based on LCQROFN for $\rho_{C Q}>1$; we get the following results.

Theorem 2. For any family of CQROFNs $\mathfrak{G}_{C Q}=\left(\mathfrak{M}_{\mathfrak{C}_{p P}} e^{i 2 \pi\left(\mathfrak{M}_{\mathfrak{C}_{I P}}\right)}, \mathfrak{N}_{\mathfrak{C}_{P P}} e^{i 2 \pi\left(\mathfrak{N}_{\mathfrak{C}_{I P}}\right)}\right)$, if $0<\rho_{C Q} \leq \mathrm{min}$ $\left\{\mathfrak{M}_{\mathfrak{C}_{R P}},\left(1-\mathfrak{N}_{\mathfrak{C}_{R P}} q_{C Q}\right)^{1 / q_{C Q}}\right\} \leq 1, \quad 0<\rho_{C Q} \leq \min \left\{\mathfrak{M}_{\mathfrak{C}_{I P}},(1-\right.$ $\left.\mathfrak{N}_{\mathfrak{C}_{I P}}^{q_{C Q}}\right)^{1 / q_{C Q}} \leq 1$ and $\rho_{C Q} \neq 1$, then

(1) $\rho_{C Q}^{\log _{\rho Q Q}} \mathfrak{E}_{C Q}=\mathfrak{S}_{C Q}$

(2) $\log _{\rho_{C Q}} \rho_{C Q}^{\mathfrak{c}_{C Q}}=\mathfrak{c}_{C Q}$

Proof

(1) By using Definition 2 and Theorem 2, we obtain

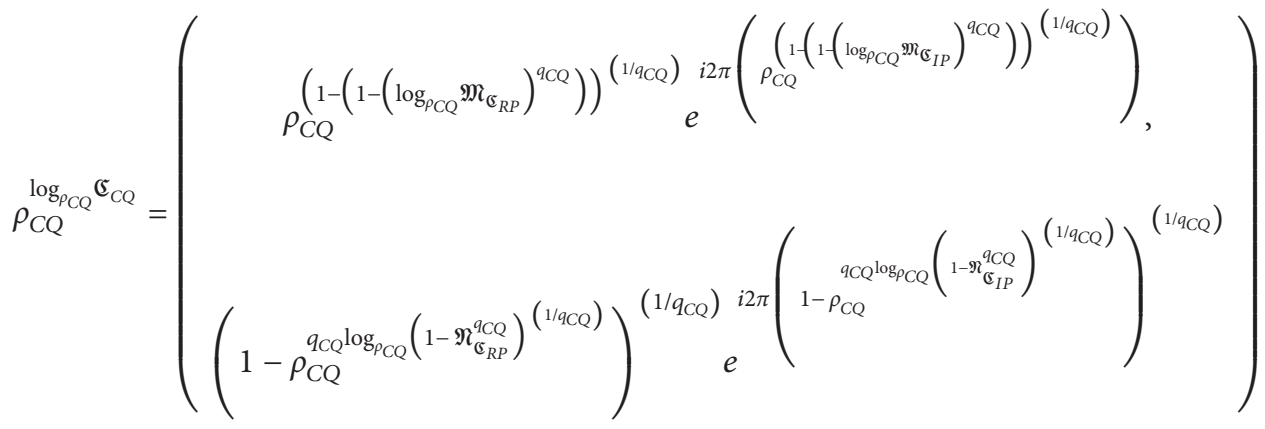

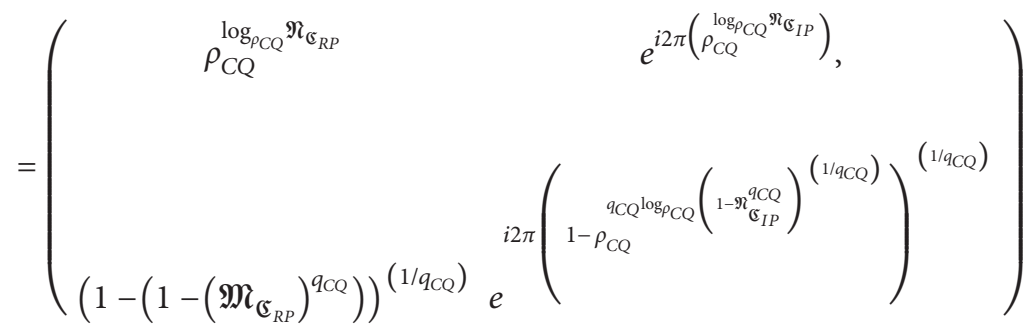

$$
\begin{aligned}
& =\left(\mathfrak{M}_{\mathfrak{C}_{R P}} e^{i 2 \pi\left(\mathfrak{M}_{\mathfrak{C}_{I P}}\right)}, \mathfrak{N}_{\mathfrak{C}_{R P}} e^{i 2 \pi\left(\mathfrak{N}_{\mathfrak{C}_{I P}}\right)}\right)=\mathfrak{C}_{C Q} .
\end{aligned}
$$


(2) By using Definition 2 and Theorem 2, we obtain

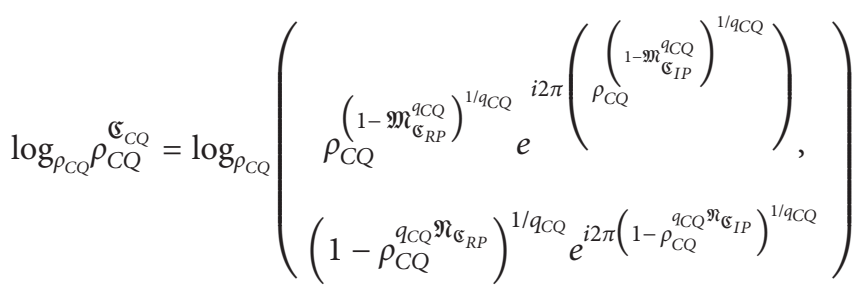

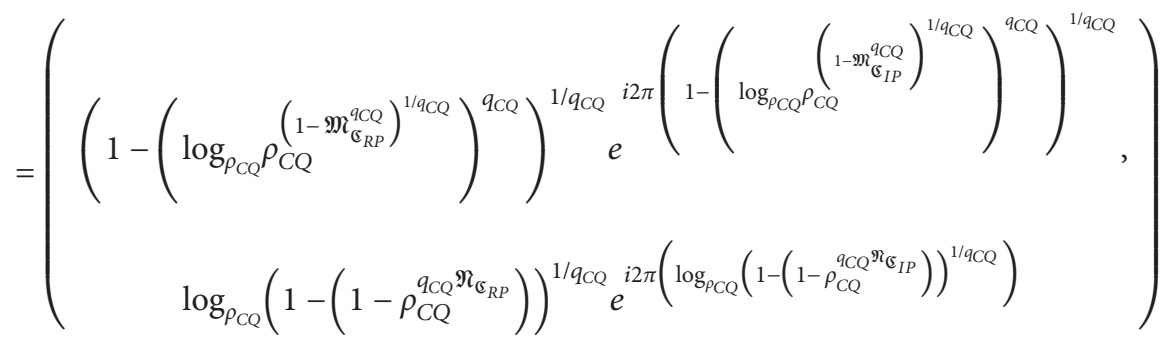

$$
\begin{aligned}
& =\left(\mathfrak{M}_{\mathfrak{C}_{R P}} e^{i 2 \pi\left(\mathfrak{M}_{\mathbb{E}_{I P}}\right)}, \mathfrak{N}_{\mathfrak{C}_{R P}} e^{i 2 \pi\left(\mathfrak{M}_{\mathbb{E}_{I P}}\right)}\right)=\mathfrak{C}_{C Q} .
\end{aligned}
$$

Theorem 3. For any family of CQROFNs Proof. Omitted.

$\mathfrak{C}_{C Q}=\left(\mathfrak{M}_{\mathfrak{C}_{R P}} e^{i 2 \pi\left(\mathfrak{M}_{\mathbb{C}_{I P}}\right)}, \mathfrak{N}_{\mathfrak{C}_{R P}} e^{i 2 \pi\left(\mathfrak{N}_{\mathbb{C}_{I P}}\right)}\right), \quad$ if $\quad 0<\rho_{C Q}$ $\leq \min _{i}\left\{\mathfrak{M}_{\mathfrak{C}_{R P-i}}, \quad\left(1-\mathfrak{N}_{\mathfrak{C}_{R P-i}}^{q_{C Q}}\right)^{1 / q_{C Q}}\right\} \leq 1, \quad 0<\rho_{C Q} \quad \leq \min _{i}$ $\left\{\mathfrak{M}_{\mathbb{C}_{I P-i}},\left(1-\mathfrak{N}_{\mathfrak{I}_{I P-i}}^{q_{C Q}}\right)^{1 / q_{C Q}}\right\} \leq 1$ and $\rho_{C Q} \neq 1$, then

(1) $\log _{\rho_{C Q}} \quad \mathfrak{夭}_{C Q-1} \oplus \log _{\rho_{C Q}} \mathfrak{夭}_{C Q-2}=\log _{\rho_{C Q}} \mathfrak{夭}_{C Q-2} \oplus \log _{\rho_{C Q}}$ $\mathfrak{c}_{\mathrm{CQ}-1}$

(2) $\left(\log _{\rho_{C Q}} \mathfrak{夭}_{\mathrm{CQ}-1} \otimes \log _{\rho_{C Q}} \mathfrak{夭}_{\mathrm{CQ}-2}\right) \otimes \log _{\rho_{\mathrm{CQ}}} \quad \mathfrak{夭}_{\mathrm{CQ}-3}=$ $\log _{\rho_{C Q}} \mathfrak{E}_{C Q-1} \otimes\left(\log _{\rho_{C Q}} \mathfrak{E}_{C Q-2} \otimes \log _{\rho_{C Q}} \mathfrak{c}_{C Q-3}\right)$

Theorem 5. For any family of CQROFNs

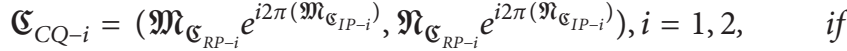
$0<\rho_{C Q} \leq \min _{i}\left\{\mathfrak{M}_{\mathbb{E}_{I P-i}},\left(1-\mathfrak{N}_{\mathfrak{C}_{I P-i}}^{q_{C Q}}\right)^{1 / q_{C Q}}\right\} \leq 1$ and $\rho_{C Q} \neq 1$ with $\mathfrak{Q}, \mathfrak{\Omega}_{1}, \mathfrak{\Omega}_{2}>0$, then

(1) $\mathfrak{L}\left(\log _{\rho_{\mathrm{CQ}}} \mathfrak{夭}_{\mathrm{CQ}-1} \oplus \log _{\rho_{\mathrm{CQ}}} \mathfrak{夭}_{\mathrm{CQ}-2}\right)=\quad \mathfrak{L} \log _{\rho_{\mathrm{CQ}}} \mathfrak{夭}_{\mathrm{CQ}-1} \oplus$ $\mathfrak{L} \log _{\rho_{C Q}} \mathfrak{E}_{C Q-2}$

Proof. Omitted.

Theorem 4. For any family of CQROFNs $\mathfrak{C}_{C Q}=\left(\mathfrak{M}_{\mathfrak{C}_{R P}} e^{i 2 \pi\left(\mathfrak{M}_{\mathbb{C}_{I P}}\right)}, \mathfrak{N}_{\mathfrak{C}_{R P}} e^{i 2 \pi\left(\mathfrak{\Re}_{\mathbb{C}_{I P}}\right)}\right)$, if $0<\rho_{C Q} \leq \min _{i}$ $\left\{\mathfrak{M}_{\mathfrak{E}_{R P-i}}\left(1-\quad \mathfrak{N}_{\mathfrak{C}_{R P-i}} q_{C Q}\right)^{1 / q_{C Q}}\right\} \leq 1, \quad 0<\rho_{C Q} \leq \min _{i}\left\{\mathfrak{M}_{\mathfrak{C}_{I P-i} ;}\right.$ $\left(1-\mathfrak{N}_{\mathfrak{C}_{I P-i}} q_{C Q}\right)^{\left.1 / q_{C Q}\right\}} \leq 1$ and $\rho_{C Q} \neq 1$, then

(1) $\left(\log _{\rho_{C Q}} \mathfrak{夭}_{\mathrm{CQ}-1} \oplus \log _{\rho_{C Q}} \mathfrak{夭}_{\mathrm{CQ}-2}\right) \oplus \log _{\rho_{\mathrm{CQ}}} \mathfrak{夭}_{\mathrm{CQ}-3}=$ $\log _{\rho_{C Q}} \mathfrak{E}_{C Q-1} \oplus\left(\log _{\rho_{C Q}} \mathfrak{E}_{\mathrm{CQ}-2} \oplus \log _{\rho_{C Q}} \mathfrak{E}_{\mathrm{CQ}-3}\right)$

(2) $\left(\log _{\rho_{C Q}} \mathfrak{夭}_{C Q-1} \otimes \log _{\rho_{C Q}} \quad \mathfrak{夭}_{C Q-2}\right) \otimes \log _{\rho_{C Q}} \mathfrak{夭}_{C Q-3}=$ $\log _{\rho_{C Q}} \mathfrak{夭}_{C Q-1} \otimes\left(\log _{\rho_{C Q}} \mathfrak{夭}_{C Q-2} \otimes \log _{\rho_{C Q}} \mathfrak{夭}_{C Q-3}\right)$

(2) $\left(\log _{\rho_{C Q}} \mathfrak{夭}_{C Q-1} \otimes \log _{\rho_{C Q}} \mathfrak{夭}_{C Q-2}\right)^{\mathfrak{Q}}=\left(\log _{\rho_{C Q}} \mathfrak{夭}_{C Q-1}\right)^{\mathfrak{Q} \otimes}$ $\left(\log _{\rho_{C Q}} \mathfrak{夭}_{C Q-2}\right)^{\mathbb{2}}$

(3) $\mathfrak{\Omega}_{1} \log _{\rho_{C Q}} \mathfrak{c}_{C Q-1} \oplus \mathfrak{\Omega}_{2} \log _{\rho_{C Q}} \mathfrak{c}_{C Q-1}=\left(\mathfrak{\Omega}_{1}+\mathfrak{\Omega}_{2}\right) \quad \log _{\rho_{C Q}}$ $\mathfrak{\mathfrak { C }}_{\mathrm{CQ}-1}$

(4) $\left(\log _{\rho_{C Q}\left(\mathfrak{\Omega}_{1}+\mathfrak{\Omega}_{2}\right)} \mathfrak{夭}_{C Q-1}\right)^{\mathfrak{Q}_{1}} \otimes\left(\log _{\rho_{C Q}} \mathfrak{\mathfrak { C }}_{C Q-1}\right)^{\mathfrak{R}_{2}}=\left(\log _{\rho_{C Q}}\right.$ $\left.\mathfrak{C}_{C Q-1}\right)\left(\Omega_{1}+\mathfrak{\Omega}_{2}\right)$

(5) $\left(\left(\log _{\rho_{C Q}} \mathfrak{夭}_{C Q-1}\right)^{\mathfrak{R}_{1}}\right)^{\mathfrak{Q}_{2}}=\left(\log _{\rho_{C Q}} \mathfrak{夭}_{C Q-1}\right)^{\mathfrak{Q}_{1} \mathfrak{R}_{2}}$

Proof. By using $\quad \mathfrak{C}_{\mathrm{CQ}-i}=\left(\mathfrak{M}_{\mathfrak{C}_{R P-i}} e^{i 2 \pi\left(\mathfrak{M}_{\mathbb{C}_{I P-i}}\right)}, \mathfrak{N}_{\mathfrak{C}_{R P-i}}\right.$ $\left.e^{i 2 \pi\left(\mathfrak{R}_{\mathbb{I}_{I P-i}}\right)}\right), i=1,2$, with Definition 2, we obtain 
8

Complexity

$$
\begin{aligned}
& \log _{\rho_{C Q}} \mathfrak{S}_{C Q-1}=\left(\begin{array}{c}
\left(1-\left(\log _{\rho_{C Q}} \mathfrak{M}_{\mathfrak{E}_{R P-1}}\right)^{q_{C Q}}\right)^{1 / q_{C Q}} e^{i 2 \pi\left(1-\left(\log _{P C Q} \mathfrak{M}_{\mathbb{G}_{I P-1}}\right)^{q C Q}\right)^{1 / 9 C Q}}, \\
\log _{\rho_{C Q}}\left(1-\mathfrak{N}_{\mathbb{C}_{R P-1}}^{q_{C Q}}\right)^{1 / q_{C Q}} e^{i 2 \pi\left(\log _{\rho_{C Q}}\left(1-\mathfrak{\Re}_{\mathbb{S}_{I P-1}}^{q_{C Q}}\right)^{1 / 9 C Q}\right)}
\end{array}\right), \\
& \log _{\rho_{C Q}} \mathbb{S}_{C Q-2}=\left(\begin{array}{c}
\left(1-\left(\log _{\rho_{C Q}} \mathfrak{M}_{\mathfrak{C}_{R P-2}}\right)^{q_{C Q}}\right)^{1 / q_{C Q}} e^{i 2 \pi\left(1-\left(\log _{P C Q} \mathfrak{M}_{\mathbb{E}_{I P-2}}\right)^{q C Q}\right)^{1 / / C Q}}, \\
\log _{\rho_{C Q}}\left(1-\mathfrak{N}_{\mathfrak{C}_{R P-2}}^{q_{C Q}}\right)^{1 / q_{C Q}} e^{i 2 \pi\left(\log _{\rho_{C Q}}\left(1-\mathfrak{\Re}_{\mathbb{C}_{I P-2}}^{q C Q}\right)^{1 / / C Q Q}\right)}
\end{array}\right) .
\end{aligned}
$$

By combining the above with the help of Definition 2, we obtain

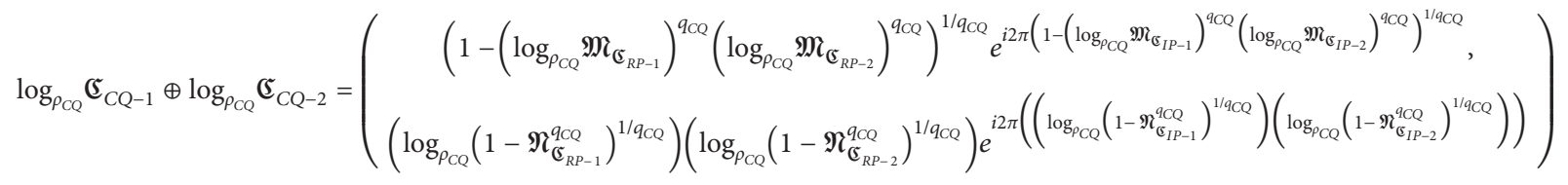

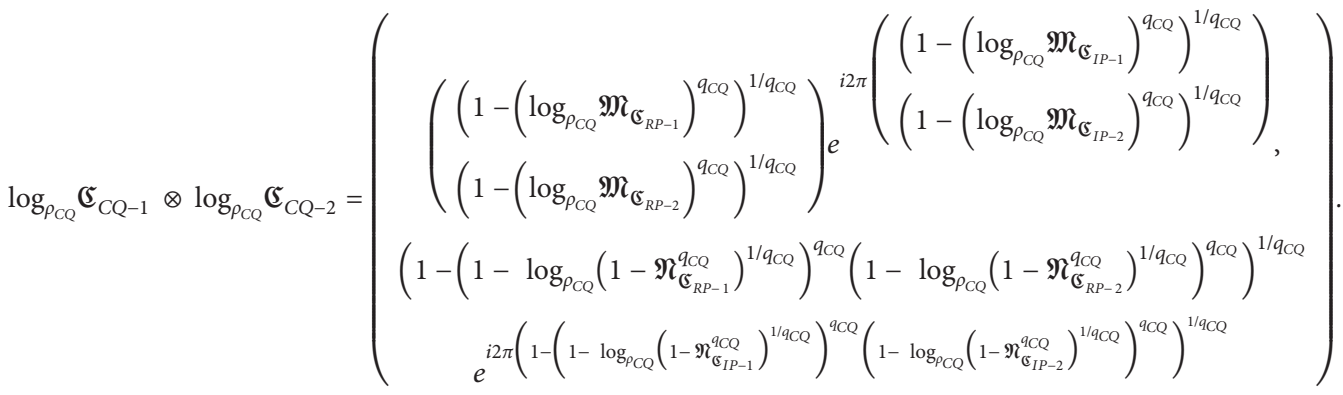

(1) For $\mathfrak{\Omega}>0$, we obtain

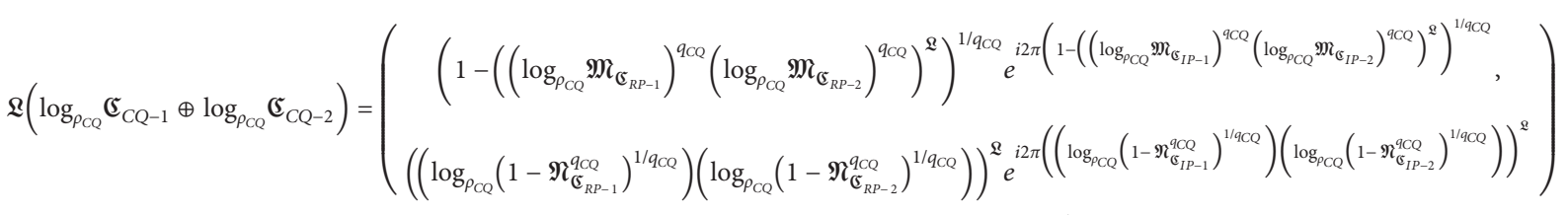

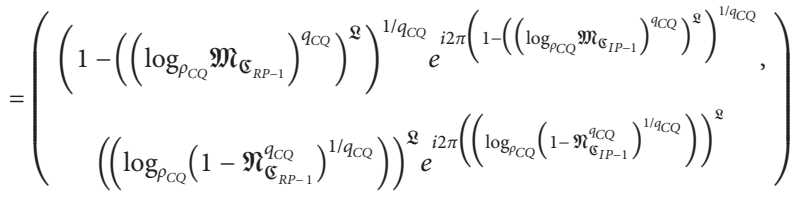

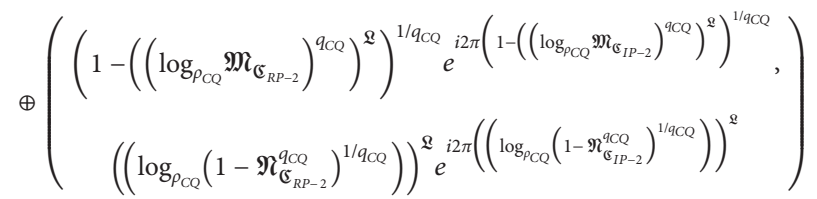

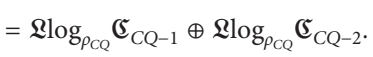


Complexity

9

(2) For $\mathfrak{Q}>0$, we obtain

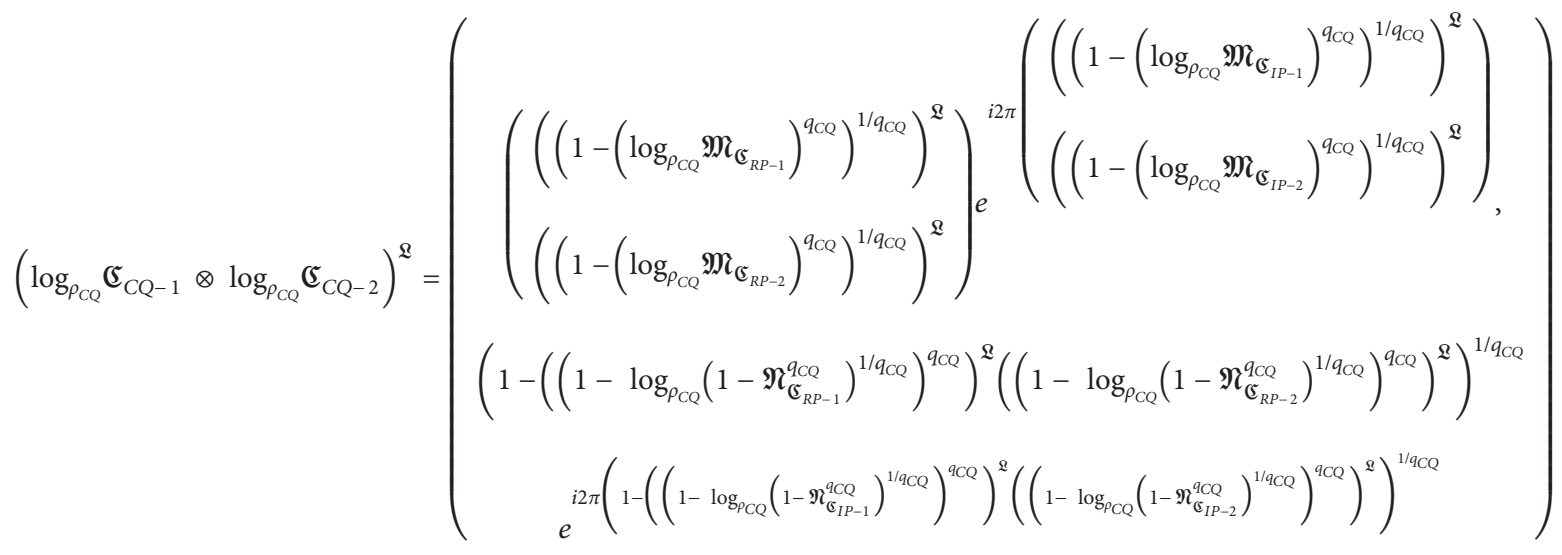

$$
\begin{aligned}
& =\left(\log _{\rho_{C Q}} \mathfrak{夭}_{C Q-1}\right)^{\mathfrak{Q}} \otimes\left(\log _{\rho_{C Q}} \mathfrak{夭}_{C Q-2}\right)^{\mathfrak{L}} .
\end{aligned}
$$

(3) For $\mathfrak{R}_{1}, \mathfrak{\Omega}_{2}>0$, we obtain

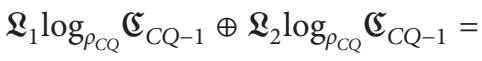

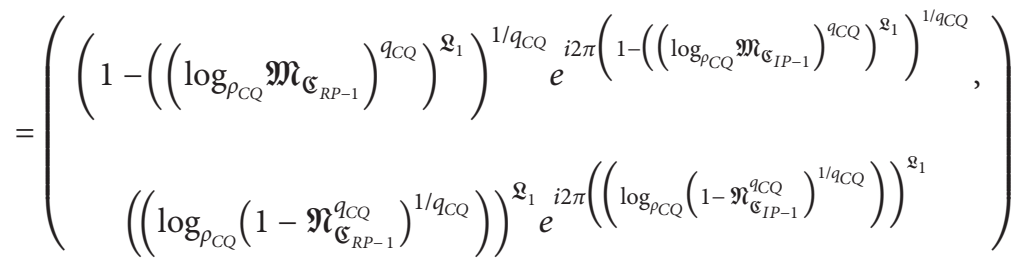

$$
\begin{aligned}
& \oplus\left(\begin{array}{c}
\left(1-\left(\left(\log _{\rho_{C Q}} \mathfrak{M}_{\mathfrak{C}_{R P-1}}\right)^{q_{C Q}}\right)^{\mathfrak{\Omega}_{2}}\right)^{1 / q_{C Q}} e^{i 2 \pi\left(1-\left(\left(\log _{\rho_{C Q}} \mathfrak{M}_{\mathfrak{C}_{I P-1}}\right)^{q_{C Q}}\right)^{\mathfrak{\Omega}_{2}}\right)^{1 / q_{C Q}}}, \\
\left(\left(\log _{\rho_{C Q}}\left(1-\mathfrak{N}_{\mathfrak{C}_{R P-1}}^{q_{C Q}}\right)^{1 / q_{C Q}}\right)\right)^{\mathfrak{\Omega}_{2}} e^{i 2 \pi\left(\left(\log _{\rho_{C Q}}\left(1-\mathfrak{N}_{\mathfrak{C}_{I P-1}}^{q_{C Q}}\right)^{1 / q_{C Q}}\right)\right)^{\mathfrak{S}_{2}}}
\end{array}\right) \\
& =\left(\begin{array}{c}
\left(1-\left(\left(\log _{\rho_{C Q}} \mathfrak{M}_{\mathfrak{C}_{R P-1}}\right)^{q_{C Q}}\right)^{\mathfrak{\Omega}_{1}+\mathfrak{\Omega}_{2}}\right)^{1 / q_{C Q}} e^{i 2 \pi\left(1-\left(\left(\log _{P C Q} \mathfrak{M}_{\mathfrak{C}_{I P-1}}\right)^{q_{C Q}}\right)^{\mathfrak{I}_{1}+\mathfrak{\Omega}_{2}}\right)^{1 / q_{C Q}}}, \\
\left(\left(\log _{\rho_{C Q}}\left(1-\mathfrak{N}_{\mathfrak{\complement}_{R P-1}}^{q_{C Q}}\right)^{1 / q_{C Q}}\right)\right)^{\mathfrak{\Omega}_{1}+\mathfrak{\Omega}_{2}} e^{i 2 \pi\left(\left(\log _{\rho_{C Q}}\left(1-\mathfrak{N}_{\mathbb{C}_{I P-1}}^{q_{C Q}}\right)^{1 / 9 C Q}\right)\right)^{\mathfrak{I}_{1}+\mathfrak{\Omega}_{2}}}
\end{array}\right) \\
& =\left(\mathfrak{Q}_{1}+\mathfrak{\Omega}_{2}\right) \log _{\rho_{C Q}} \mathfrak{\mathfrak { S }}_{\mathrm{CQ}-1} .
\end{aligned}
$$


10

Complexity

(4) For $\mathfrak{l}_{1}, \mathfrak{Q}_{2}>0$, we obtain

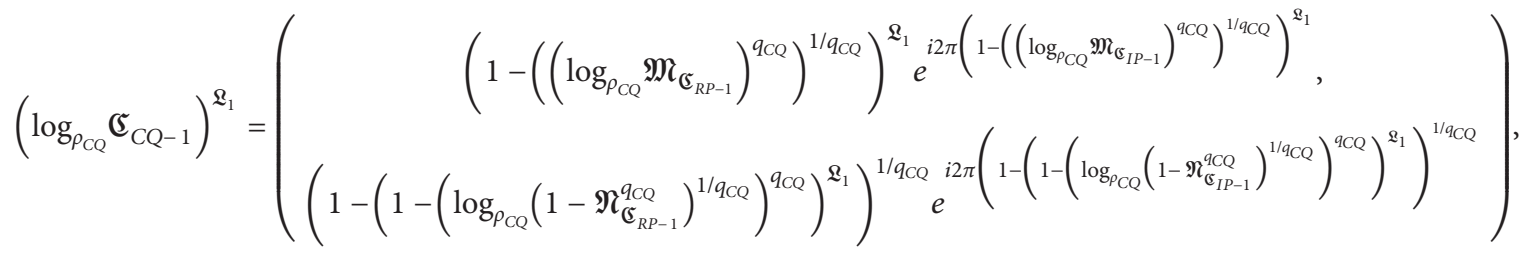

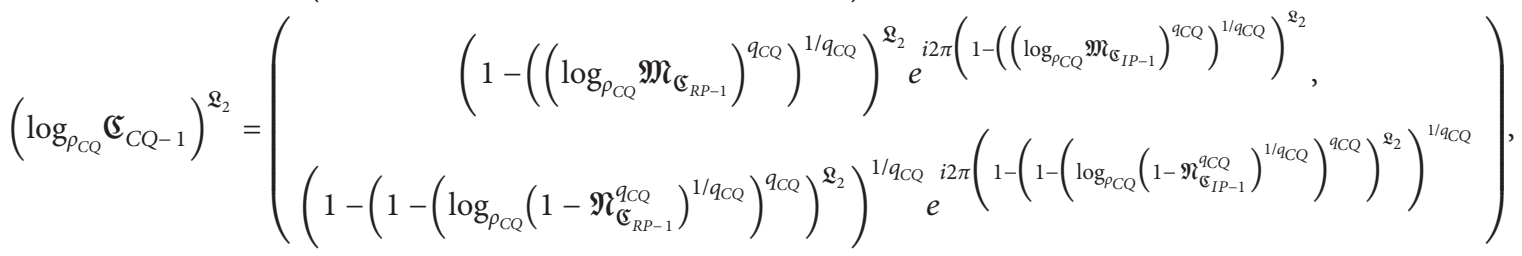

and then

$$
\begin{aligned}
& \left(\log _{\rho_{C Q}} \mathfrak{夭}_{C Q-1}\right)^{\mathfrak{Q}_{1}} \otimes\left(\log _{\rho_{C Q}} \mathfrak{夭}_{C Q-1}\right)^{\mathfrak{R}_{2}}
\end{aligned}
$$

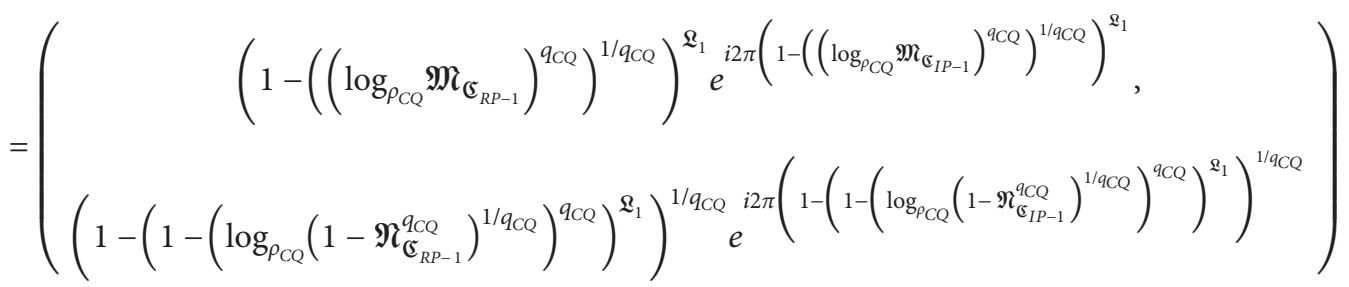

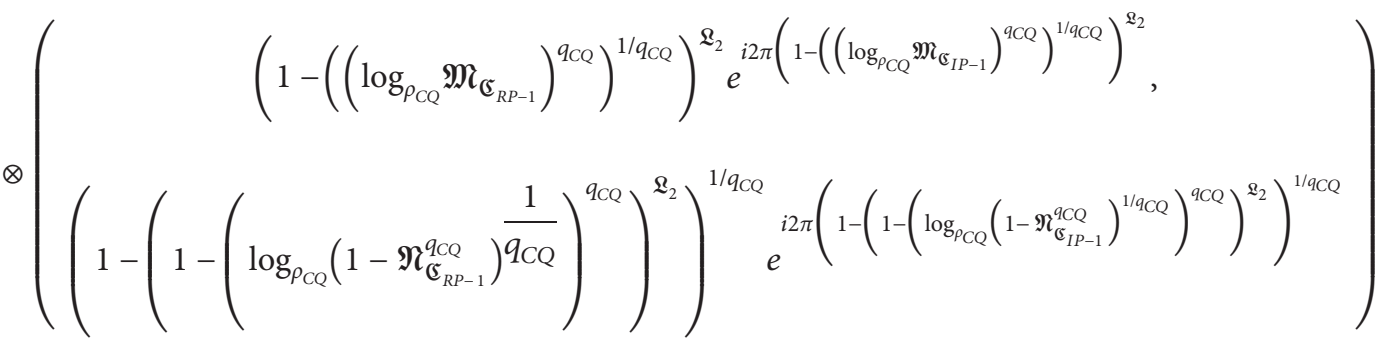

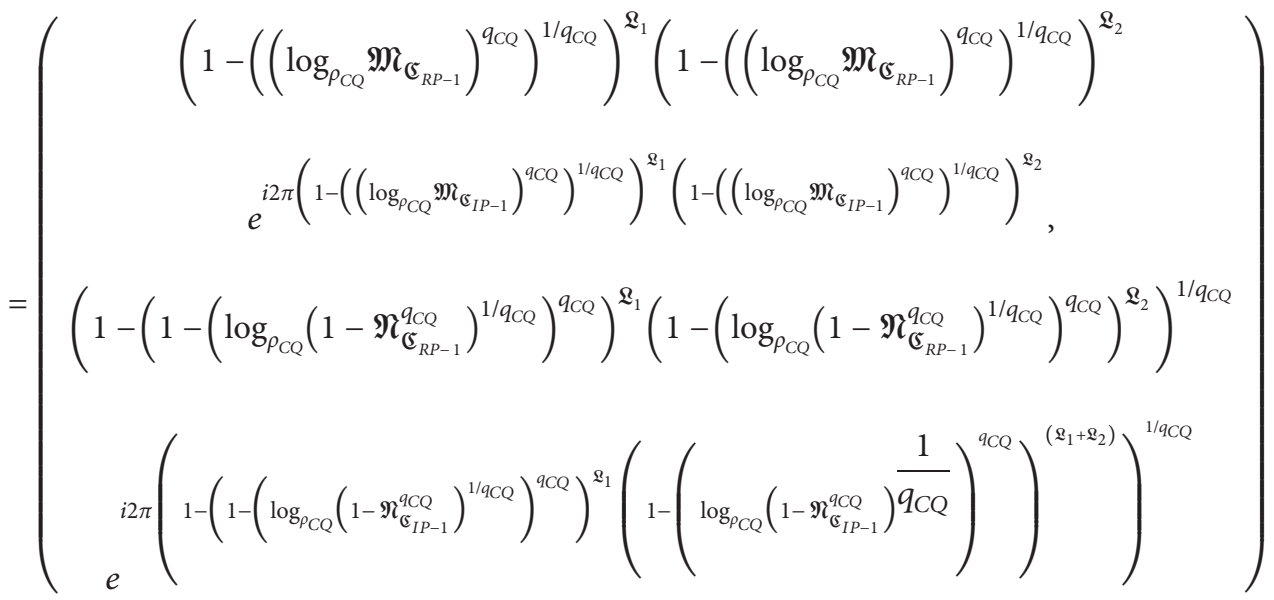

$$
\begin{aligned}
& =\left(\log _{\rho_{C Q}} \overleftarrow{夭}_{C Q-1}\right)^{\left(\Omega_{1}+\Omega_{2}\right)} \text {. }
\end{aligned}
$$


(5) For $\mathfrak{Q}_{1}, \mathfrak{\Omega}_{2}>0$, we obtain

$$
\begin{aligned}
& \left(\left(\log _{\rho_{C Q}} \mathfrak{夭}_{C Q-1}\right)^{\mathfrak{Q}_{1}}\right)^{\mathfrak{Q}_{2}}
\end{aligned}
$$

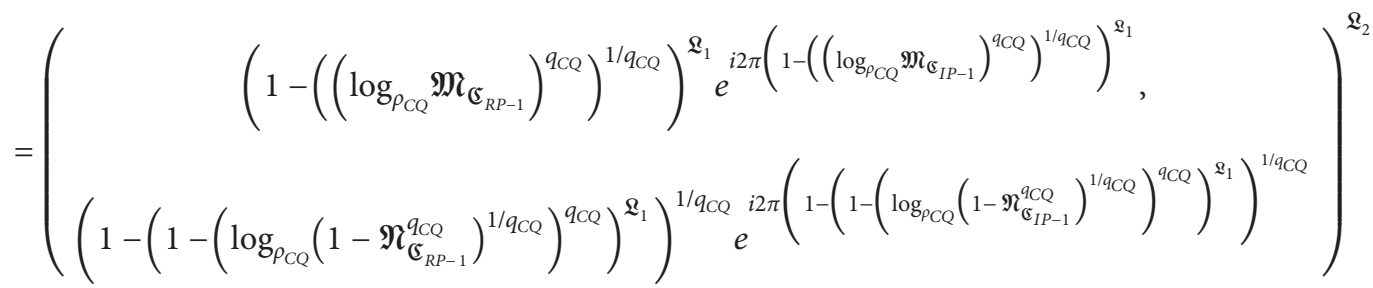

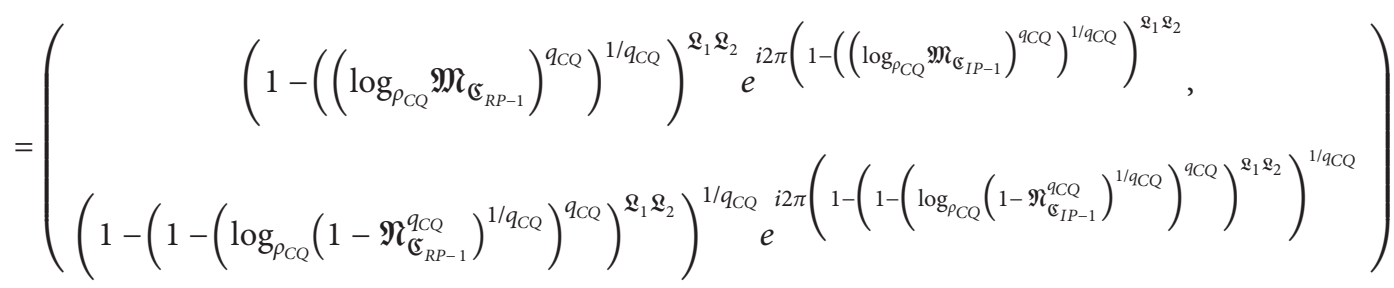

$$
\begin{aligned}
& =\left(\log _{\rho_{C Q}} \mathfrak{\complement}_{C Q-1}\right)^{\mathfrak{R}_{1} \mathfrak{R}_{2}} \text {. }
\end{aligned}
$$

Theorem 6. For any family of CQROFNs $\mathfrak{C}_{C Q-i}=\left(\mathfrak{M}_{\mathfrak{C}_{R P-i}} e^{i 2 \pi\left(\mathfrak{M}_{\mathfrak{G}_{I P-i}}\right)}, \mathfrak{N}_{\mathfrak{C}_{R P-i}} e^{i 2 \pi\left(\mathfrak{M}_{\mathfrak{G}_{I P-i}}\right)}\right), \quad i=1,2, \quad$ if $0<\rho_{C Q-1} \leq \rho_{C Q-2} \leq \min _{i}\left\{\mathfrak{M}_{\mathbb{E}_{R P-i},}\left(1-\mathfrak{N}_{\mathbb{E}_{R P-i}}^{q_{\mathrm{CQ}}}\right)^{1 / q_{\mathrm{CQ}}}\right\} \leq 1, \quad 0$ $<\rho_{C Q-1} \leq \rho_{C Q-2} \leq \min _{i}\left\{\mathfrak{M}_{\mathbb{E}_{I P-i}},\left(1-\mathfrak{N}_{\mathfrak{E}_{I P-i}}^{q_{C P-i}}\right)^{1 / q_{C Q}}\right\} \leq 1$, and $\rho_{C Q-1}, \rho_{C Q-2} \neq 1$, then $\log _{\rho_{\mathrm{CQ}-1}} \mathfrak{S}_{\mathrm{CQ}} \leq \log _{\rho_{\mathrm{CQ}-2}} \mathfrak{夭}_{\mathrm{CQ}}$ and $\log _{\rho_{\mathrm{CQ}-1}} \mathfrak{G}_{C Q} \geq \log _{\rho_{\mathrm{CQ}-2}} \mathfrak{E}_{C Q}, \quad$ for $\quad 0<\left(1 / \rho_{\mathrm{CQ}-1}\right) \quad \leq=$ $\left(1 / \rho_{C Q-2}\right) \leq \min _{i}\left\{\mathfrak{M}_{\mathfrak{E}_{R P-i}},\left(1-\mathfrak{N}_{\mathfrak{C}_{R P-i}}^{q_{C Q}}\right) \quad 1 / q_{C Q}\right\} \quad \leq 1, \quad 0<$ $\left(1 / \rho_{C Q-1}\right) \leq\left(1 / \rho_{C Q-2}\right) \leq \min _{i}\left\{\mathfrak{M}_{\mathfrak{C}_{I P-i},}\left(1-\mathfrak{N}_{\mathfrak{C}_{I P-i}}^{q_{C Q}}\right)\left(1 / q_{C Q}\right)\right\}$ $\leq 1$, and $\rho_{\mathrm{CQ}-1}, \rho_{\mathrm{CQ}-2} \neq 1$.

Proof. By using Definition 2, we obtain

$$
\begin{aligned}
& \log _{\rho_{C Q}-1} \mathbb{C}_{C Q}=\left(\begin{array}{c}
\left(1-\left(\log _{\rho_{C Q-1}} \mathfrak{M}_{\mathfrak{C}_{R P}}\right)^{q_{C Q}}\right)^{1 / q_{C Q}} e^{i 2 \pi\left(1-\left(\log _{\rho_{C Q-1}} \mathfrak{M}_{\mathfrak{C}_{I P}}\right)^{q_{C Q}}\right)^{1 / 9 C Q}}, \\
\left(\log _{\rho_{C Q-1}}\left(1-\mathfrak{N}_{\mathfrak{C}_{R P}}^{q_{C Q}}\right)^{1 / q_{C Q}}\right) e^{i 2 \pi\left(\log _{\rho_{C Q-1}}\left(1-\mathfrak{N}_{\mathbb{C}_{I P}}^{q C Q}\right)^{1 / 9 C Q}\right)}
\end{array}\right), \\
& \log _{\rho_{C Q}-2} \mathbb{C}_{C Q}=\left(\begin{array}{c}
\left(1-\left(\log _{\rho_{C Q-2}} \mathfrak{M}_{\mathbb{C}_{R P}}\right)^{q_{C Q}}\right)^{1 / q_{C Q}} e^{i 2 \pi\left(1-\left(\log _{\rho_{C Q-2}} \mathfrak{M}_{\mathbb{C}_{I P}}\right)^{q C Q}\right)^{1 / q C Q}}, \\
\left(\log _{\rho_{C Q-2}}\left(1-\mathfrak{N}_{\mathbb{C}_{R P}}^{q_{C Q}}\right)^{1 / q_{C Q}}\right) e^{i 2 \pi\left(\log _{\rho_{C Q-2}}\left(1-\mathfrak{M}_{\mathbb{I}_{I P}}^{q_{C Q}}\right)^{1 / q_{C Q}}\right)}
\end{array}\right) .
\end{aligned}
$$

When $0<\rho_{C Q-1} \leq \rho_{C Q-2} \leq \min _{i}\left\{\mathfrak{M}_{\mathfrak{E}_{I P-i},} \quad\left(1-\mathfrak{N}_{\mathbb{E}_{I P-i}}^{q_{C Q}}\right)\right.$ $1 / q_{C Q} \leq 1 \quad$ and $\quad \rho_{C Q-1}, \rho_{C Q-2} \neq 1$, then (1$\left.\left(\log _{\rho_{C Q-1}} \mathfrak{M}_{\mathfrak{C}_{R P}}\right)^{q_{C Q}}\right) \geq\left(1-\left(\log _{\rho_{C Q-2}} \mathfrak{M}_{\mathfrak{C}_{R P}}\right)^{q_{C Q}}\right),\left(1-\left(\log _{\rho_{C Q-1}}\right.\right.$ $\left.\left.\mathfrak{M}_{\mathfrak{C}_{I P}}\right)^{q_{C Q}}\right) \geq\left(1-\left(\log _{\rho_{C Q-2}} \mathfrak{M}_{\mathfrak{C}_{I P}}\right)^{q_{C Q}}\right)$ and $\log _{\rho_{C Q-1}}(1-\mathfrak{N}$ $\left.\begin{array}{l}q_{C Q} \\ \mathfrak{E}_{R P}\end{array}\right)^{1 / q_{C Q}} \quad \leq \log _{\rho_{C Q-2}} \quad\left(1-\mathfrak{N}_{\mathfrak{C}_{R P}}^{q_{C Q}}\right) \quad 1 / q_{C Q}, \log _{\rho_{C Q-1}}$ $\left(1-\mathfrak{N}_{\mathfrak{G}_{I P}}^{q_{\mathrm{CQ}}}\right)^{1 / q_{\mathrm{CQ}}} \leq \log _{\rho_{\mathrm{CQ}-2}}\left(1-\mathfrak{N}_{\mathfrak{G}_{I P}}^{q_{\mathrm{CQ}}}\right)^{1 / q_{\mathrm{CQ}}}$, and then $\log _{\rho_{\mathrm{CQ}^{-1}}} \mathfrak{\mathfrak { c }}_{C Q} \geq \log _{\rho_{\mathrm{CQ}^{-2}}} \mathfrak{\mathfrak { c }}_{C Q}$. Moreover, $\rho_{\mathrm{CQ}-1}, \rho_{\mathrm{CQ}-2}>1$ and $\rho_{C Q-1} \leq \rho_{C Q-2}$; then, $\left.0<\left(1 / \rho_{C Q-1}\right) \leq\right]\left(1 / \rho_{C Q-2}\right) \leq \min _{i} \quad\left\{\mathfrak{M}_{\mathfrak{C}_{R P-i}}\left(1-\mathfrak{N}_{\mathfrak{G}_{R P-i}}^{q_{\mathrm{CQ}}}\right)\right.$ $1 / q_{C Q} \leq 1, \quad 0<\quad\left(1 / \rho_{C Q-1}\right) \leq\left(1 / \rho_{C Q-2}\right) \leq \min _{i}$ $\left\{\mathfrak{M}_{\mathbb{C}_{I P-i}},\left(1-\mathfrak{N}_{\mathfrak{C}_{I P-i}}^{q_{C Q}}\right)^{1 / q_{C Q}}\right\} \leq 1$, and $\rho_{C Q-1}, \rho_{C Q-2} \neq 1$; then, we obtain $\log _{\rho_{\mathrm{CQ}}-1} \mathfrak{夭}_{\mathrm{CQ}} \leq \log _{\rho_{\mathrm{CQ}-2}} \mathfrak{夭}_{\mathrm{CQ}}$.

Theorem 7. For any family of CQROFNs

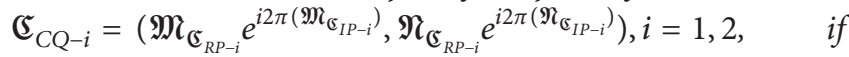


$\mathfrak{E}_{C Q-1} \leq \mathfrak{C}_{C Q-2}$, that is, $\quad \mathfrak{M}_{\mathfrak{E}_{R P-1}} \leq \mathfrak{M}_{\mathfrak{C}_{R P-2}}, \mathfrak{M}_{\mathfrak{C}_{I P-1}} \leq \mathfrak{M}_{\mathfrak{C}_{I P-2}}$, $\mathfrak{N}_{\mathfrak{C}_{R P-1}} \geq \mathfrak{N}_{\mathfrak{C}_{R P-2}}$, and $\quad \mathfrak{N}_{\mathfrak{C}_{I P-1}} \geq \mathfrak{N}_{\mathfrak{C}_{I P-2}}, \quad 0<\rho_{C Q} \leq \mathrm{min}$ ${ }_{i}\left\{\mathfrak{M}_{\mathfrak{E}_{R P-i},}\left(1-\mathfrak{N}_{\mathfrak{C}_{R P-i}}^{q_{C Q}}\right) \quad\left(1 / q_{C Q}\right)\right\} \leq 1, \quad 0<\rho_{C Q} \leq \min _{i}\left\{\mathfrak{M}_{\mathfrak{E}_{I P-i}}\right.$, $\left(1-\mathfrak{N}_{\mathfrak{C}_{I P-i}}^{q_{C Q}}\right)^{1 / q_{C Q}} \leq 1$, and $\rho_{C Q} \neq 1$, then $\log _{\rho_{C Q}} \mathfrak{夭}_{C Q-1} \leq$ $\log _{\rho_{C Q}} \mathfrak{r}_{C Q-2}$.

Proof. Omitted.

\section{Complex q-Rung Orthopair Fuzzy Aggregation Operators by Using LOLs}

By using the novel of LOLs, the theory of LCQROFWA and LCQROFWG operators is investigated. Let the family of CQROFSs be denoted by $\Xi$.

Definition 6. For any family of CQROFNs $\mathfrak{C}_{C Q-i}=\left(\mathfrak{M}_{\mathfrak{C}_{R P-i}} e^{i 2 \pi\left(\mathfrak{M}_{\mathfrak{I}_{I P-i}}\right)}, \mathfrak{N}_{\mathfrak{C}_{R P-i}} e^{i 2 \pi\left(\mathfrak{M}_{\mathfrak{I}_{I P-i}}\right)}\right), i=1,2, \ldots, \widetilde{n}$, if $\left.0<\rho_{C Q} \leq \min _{i}\left\{\mathfrak{M} \quad \mathfrak{E}_{R P-i},\left(1-\mathfrak{N}_{\mathfrak{C}_{R P-i}} q_{C Q}\right)^{(1 /} \quad q_{C Q}\right)\right\} \leq 1$,

$\operatorname{LCQROFWA}\left(\mathfrak{夭}_{\mathrm{CQ-1}}, \mathfrak{夭}_{\mathrm{CQ-2}-2}, \ldots, \mathfrak{夭}_{\mathrm{CQ}-\tilde{n}}\right)$

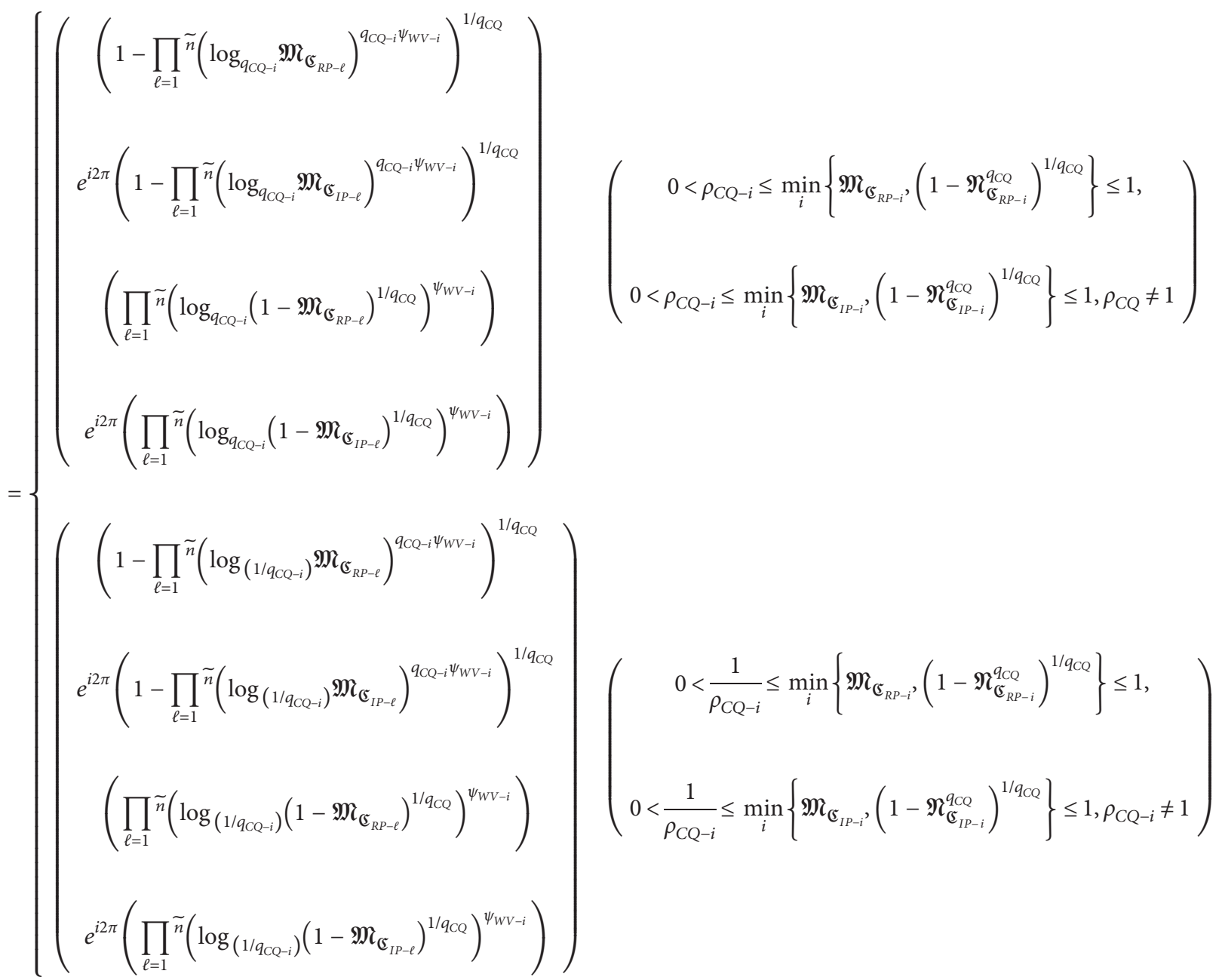

$0<\rho_{C Q} \leq \min _{i}\left\{\mathfrak{M}_{\mathbb{C}_{I P-i}},\left(1-\mathfrak{N}_{\mathfrak{C}_{I P-i}}^{q_{C Q}}\right)^{1 / q_{C Q}}\right\} \leq 1$, and $\rho_{C Q} \neq 1$, then the LCQROFWA operators are demonstrated by

LCQROFWA: $\Xi^{n} \longrightarrow \Xi$, by

$$
\begin{aligned}
\operatorname{LCQROFWA} & \left(\mathfrak{夭}_{C Q-1}, \mathfrak{夭}_{C Q-2}, \ldots, \mathfrak{夭}_{C Q-\tilde{n}}\right)=\Psi_{W V-1} \log _{\rho_{C Q-1}} \\
& \mathfrak{夭}_{C Q-1} \oplus \Psi_{W V-2} \log _{\rho_{C Q-2}} \mathfrak{夭}_{C Q-2} \oplus \ldots \\
\oplus & \Psi_{W V-\tilde{n}} \log _{\rho_{C Q-\tilde{n}}} \mathfrak{c}_{C Q-\widetilde{n}},
\end{aligned}
$$

where LCQROFWA is a logarithmic CQROFWA operator with weight vector $\Psi_{W V}=\left(\Psi_{W V-1}, \Psi_{W V-2}, \ldots, \Psi_{W V-\tilde{n}}\right)^{T}$ and $\sum_{i=1}^{n} \Psi_{W V-i}=1$.

Theorem 8. For any family of CQROFNs, $\mathfrak{E}_{C Q-i}=\left(\mathfrak{M}_{\mathfrak{C}_{R P-i}} e^{i 2 \pi\left(\mathfrak{M}_{\mathfrak{G}_{I P-i}}\right)}, \mathfrak{N}_{\mathbb{C}_{R P-i}} e^{i 2 \pi\left(\mathfrak{N}_{\mathbb{C}_{I P-i}}\right)}\right), i=1,2, \ldots, \widetilde{n}$, and by using equation (21), we obtain 
Complexity

13

Proof. By employing the mathematical induction (MI), we resolved equation (22) for $\tilde{n}$ for $0<\rho_{C Q-i} \leq \min _{i}$ $\left\{\mathfrak{M}_{\mathfrak{C}_{R P-i}},\left(1-\mathfrak{N}_{\mathfrak{C}_{R P-i}}^{q_{C Q}}\right)^{1 / q_{C Q}}\right\} \leq 1 \quad$ and $\quad 0<\rho_{C Q-i} \leq \min _{i}$
$\left\{\mathfrak{M}_{\mathfrak{C}_{I P-i}},\left(1-\mathfrak{N}_{\mathfrak{C}_{I P-i}}^{q_{C Q}}\right)^{1 / q_{C Q}}\right\} \leq 1, \rho_{C Q} \neq 1 \quad$ based $\quad$ on
$\mathfrak{C}_{C Q-i}=\left(\mathfrak{M}_{\mathfrak{C}_{R P-i}} e^{i 2 \pi\left(\mathfrak{M}_{\mathfrak{C}_{I P-i}}\right)}, \mathfrak{N}_{\mathfrak{C}_{R P-i}} e^{i 2 \pi\left(\mathfrak{N}_{\mathfrak{C}_{I P-i}}\right)}\right), i=1,2, \ldots, \widetilde{n}$ with rules $0 \leq \mathfrak{M}_{\mathfrak{C}_{R P}}^{q_{C Q}}(\widetilde{x})+\mathfrak{N}_{\mathfrak{C}_{R P}}^{q_{C Q}}(\widetilde{x}) \leq 1 \quad$ and

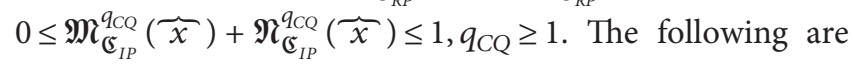
the cases.

Case 1. For $\widetilde{n}=2$, we obtain

$$
\begin{aligned}
& \operatorname{LCQROFWA}\left(\mathfrak{⿰}_{C Q-1}, \mathfrak{⿰}_{C Q-2}\right)=\Psi_{W V-1} \log _{\rho_{C Q-1}} \mathfrak{⿰}_{C Q-1} \oplus \Psi_{W V-2} \log _{\rho_{C Q-2}} \mathfrak{c}_{C Q-2} \\
& =\left(\begin{array}{c}
\left(1-\left(\log _{\rho_{C Q-1}} \mathfrak{M}_{\mathfrak{C}_{R P-1}}\right)^{\Psi_{W V-1} q_{C Q}}\right)^{1 / q_{C Q}} e^{i 2 \pi\left(1-\left(\log _{\rho_{C Q-1}} \mathfrak{M}_{\mathbb{C}_{I P-1}}\right)^{\Psi_{W V-1} q_{C Q}}\right)^{1 / q C Q}}, \\
\left(\log _{\rho_{C Q-1}}\left(1-\mathfrak{N}_{\mathfrak{C}_{R P-1}}^{q_{C Q}}\right)^{1 / q_{C Q}}\right)^{\Psi_{W V-1}} e^{i 2 \pi\left(\log _{P_{C Q-1}}\left(1-\mathfrak{N}_{\mathbb{C}_{I P-1}}^{q_{C Q}}\right)^{1 / q_{C Q}}\right)^{\Psi_{W V-1}}}
\end{array}\right) \\
& \oplus\left(\begin{array}{c}
\left(1-\left(\log _{\rho_{C Q-2}} \mathfrak{M}_{\mathfrak{C}_{R P-2}}\right)^{\Psi_{W V-2} q_{C Q}}\right)^{1 / q_{C Q}} e^{i 2 \pi\left(1-\left(\log _{\rho_{C Q-2}} \mathfrak{M}_{\mathbb{E}_{I P-2}}\right)^{\Psi_{W V-2} q C Q}\right)^{1 / q C Q}}, \\
\left(\log _{\rho_{\mathrm{CQ}-2}}\left(1-\mathfrak{N}_{\mathfrak{C}_{R P-2}}^{q_{C Q}}\right)^{1 / q_{C Q}}\right)^{\Psi_{W V-2}} e^{i 2 \pi\left(\log _{\rho_{C Q-2}}\left(1-\mathfrak{N}_{\mathfrak{C}_{I P-2}}^{q_{C Q}}\right)^{1 / q_{C Q}}\right)^{\Psi_{W V-2}}}
\end{array}\right)
\end{aligned}
$$

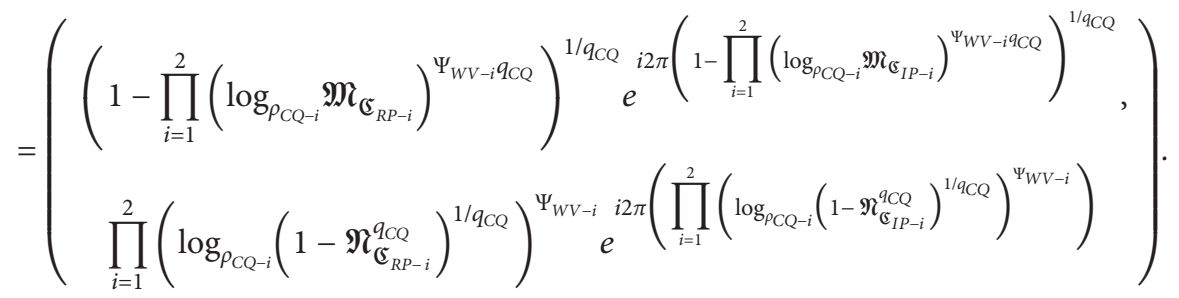

Case 2. Result holds for $\widetilde{n}=\ell$; now for $\widetilde{n}=\ell+1$, we obtain

$$
\begin{aligned}
& \operatorname{LCQROFWA}\left(\mathfrak{夭}_{C Q-1}, \mathfrak{夭}_{C Q-2}, \ldots, \mathfrak{夭}_{C Q-\ell+1}\right)=\Psi_{W V-\ell} \log _{\rho_{\mathrm{CQ}-\ell}} \mathfrak{夭}_{C Q-\ell} \oplus \Psi_{W V-\ell+1} \log _{\rho_{\mathrm{CQ}-\ell+1}} \mathfrak{夭}_{C Q-\ell+1}
\end{aligned}
$$

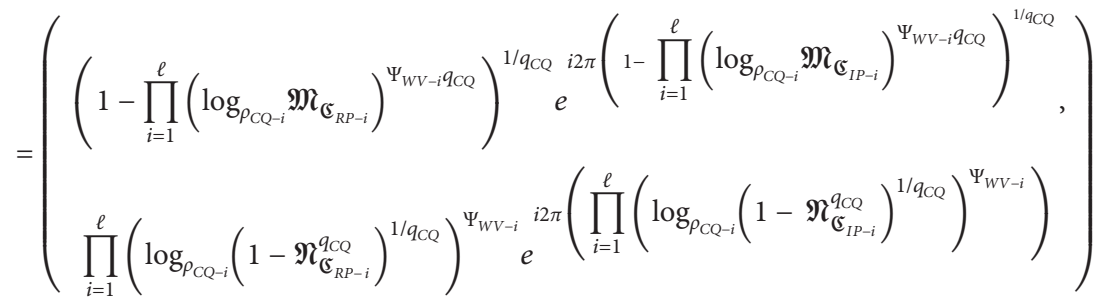

$$
\begin{aligned}
& \oplus\left(\begin{array}{c}
\left(1-\left(\log _{\rho_{C Q-\ell+1}} \mathfrak{M}_{\mathfrak{C}_{R P-\ell+1}}\right)^{\Psi_{W V-\ell+1} q_{C Q}}\right)^{1 / q_{C Q}} e^{i 2 \pi\left(1-\left(\log _{\rho_{C Q-\ell+1}} \mathfrak{M}_{\mathbb{C}_{I P-\ell+1}}\right)^{\Psi_{W V-\ell+1} q_{C Q}}\right)^{1 / q_{C Q}}}, \\
\left(\log _{\rho_{C Q-\ell+1}}\left(1-\mathfrak{N}_{\mathfrak{C}_{R P-\ell+1}}^{q_{C Q}}\right)^{1 / q_{C Q}}\right)^{\Psi_{W V-\ell+1}} e^{i 2 \pi\left(\log _{\rho_{C Q-\ell+1}}\left(1-\mathfrak{M}_{\mathbb{C}_{I P-\ell+1}}^{q_{C Q}}\right)^{1 / q_{C Q}}\right)^{\Psi_{W V-\ell+1}}}
\end{array}\right) \\
& =\left(\begin{array}{c}
\left(1-\prod_{i=1}^{\ell+1}\left(\log _{\rho_{C Q-i}} \mathfrak{M}_{\mathfrak{C}_{R P-i}}\right)^{\Psi_{W V-i} q_{C Q}}\right)^{1 / q_{C Q}} e^{i 2 \pi\left(1-\prod_{i=1}^{\ell+1}\left(\log _{\rho_{C Q Q-i}} \mathfrak{M}_{\mathfrak{G}_{I P-i}}\right)^{\Psi_{W V-i} q_{C Q}}\right)^{1 / q_{C Q}}}, \\
\left.\prod_{i=1}^{\ell+1}\left(\log _{\rho_{C Q-i}}\left(1-\mathfrak{N}_{\mathfrak{C}_{R P-i}}^{q_{C Q}}\right)^{1 / q_{C Q}}\right)^{\Psi_{W V-i}} e^{i 2 \pi\left(\prod_{i=1}^{\ell+1}\left(\log _{\rho_{C Q-i}}\left(1-\mathfrak{M}_{\mathbb{S}_{I P-i}}^{q_{C Q}}\right)^{1 / q_{C Q}}\right)^{\Psi_{W V-i}}\right.}\right)
\end{array}\right) .
\end{aligned}
$$


Hence, equation (22) is held for all $\tilde{n}$. Moreover, if based on $\mathfrak{E}_{C Q-i}=\left(\mathfrak{M}_{\mathfrak{E}_{R P-i}} e^{i 2 \pi\left(\mathfrak{M}_{\mathbb{E}_{I P-i}}\right)}, \mathfrak{N}_{\mathfrak{E}_{R P-i}} e^{i 2 \pi\left(\mathfrak{M}_{\mathbb{E}_{I P-i}}\right)}\right)$, $0<\left(1 / \rho_{C Q-i}\right) \leq \min _{i}\left\{\mathfrak{M}_{\mathbb{C}_{R P-i}}\left(1-\mathfrak{N}_{\mathbb{C}_{R P-i}}^{q_{C Q}}\right)^{1 / q_{C Q}}\right\} \leq 1 \quad$ and $\quad i=1,2, \ldots, \widetilde{n}$, with rules $0 \leq \mathfrak{M}_{\mathbb{C}_{R P}}^{q_{C Q}}(\widetilde{x})+\mathfrak{N}_{\mathbb{C}_{R P}}^{q_{C Q}}(\widetilde{x}) \leq 1$ $0<\left(1 / \rho_{C Q-i}\right) \leq \min _{i}\left\{\mathfrak{M}_{\mathbb{E}_{I P-i}},\left(1-\mathfrak{N}_{\mathfrak{G}_{I P-i}}^{q_{C Q}}\right)^{1 / q_{C Q}}\right\} \leq 1, \rho_{C Q} \neq 1, \quad$ and $0 \leq \mathfrak{M}_{\mathfrak{G}_{I P}}^{q_{C Q}}(\widetilde{x})+\mathfrak{N}_{\mathfrak{G}_{I P}}^{q_{C Q}}(\widetilde{x}) \leq 1, q_{C Q} \geq 1$, then

$$
\begin{aligned}
& \operatorname{LCQROFWA}\left(\mathfrak{夭}_{\mathrm{CQ-1}}, \mathfrak{夭}_{\mathrm{CQ-2}}, \ldots, \mathfrak{夭}_{\mathrm{CQ}-\tilde{n}}\right)
\end{aligned}
$$

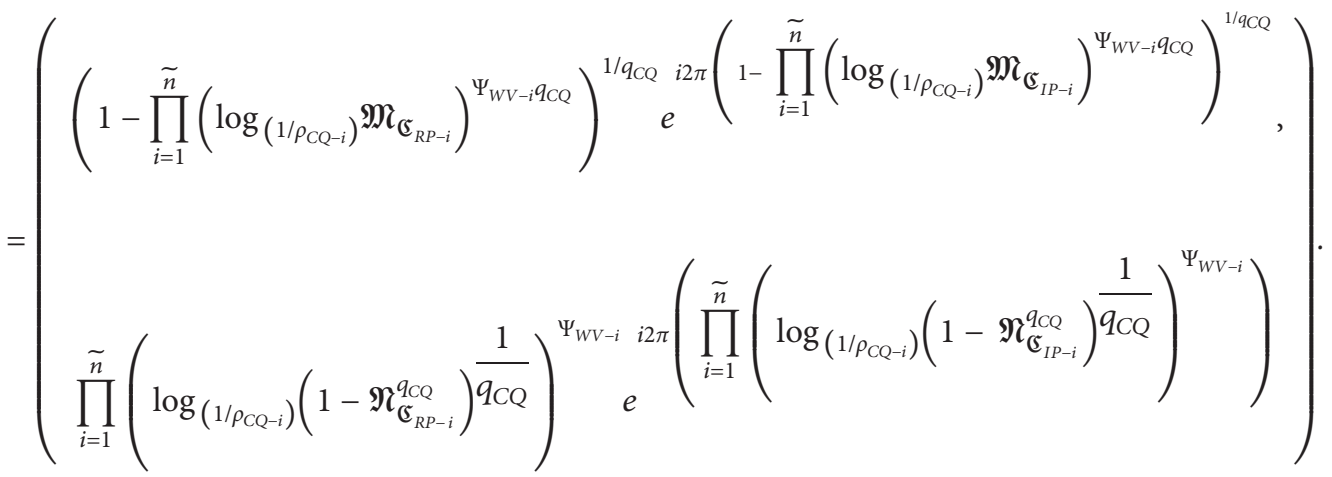

The aggregated value is again CQROFN.

$$
\begin{aligned}
& \text { Remark 1. When } \rho_{C Q-1}=\rho_{C Q-2}=\cdots=\rho_{C Q-\widetilde{n}}=\rho_{C Q}, \\
& 0<\rho_{C Q-i} \leq \min _{i}\left\{\mathfrak{M}_{\mathbb{C}_{R P-i},}\left(1-\mathfrak{N}_{\mathfrak{C}_{R P-i}}^{q_{C Q}}\right)^{1 / q_{C Q}}\right\} \leq 1, \quad \text { and } \\
& 0<\rho_{C Q-i} \leq \min _{i}\left\{\mathfrak{M}_{\mathfrak{C}_{I P-i},}\left(1-\mathfrak{N}_{\mathfrak{G}_{I P-i}}^{q_{C Q}}\right)^{1 / q_{C Q}}\right\} \leq 1, \rho_{C Q} \neq 1 \text {, then }
\end{aligned}
$$

$$
\begin{aligned}
& \operatorname{LCQROFWA}\left(\mathfrak{夭}_{\mathrm{CQ-1}}, \mathfrak{夭}_{\mathrm{CQ-2}-2}, \ldots, \mathfrak{夭}_{\mathrm{CQ}-\tilde{n}}\right)
\end{aligned}
$$

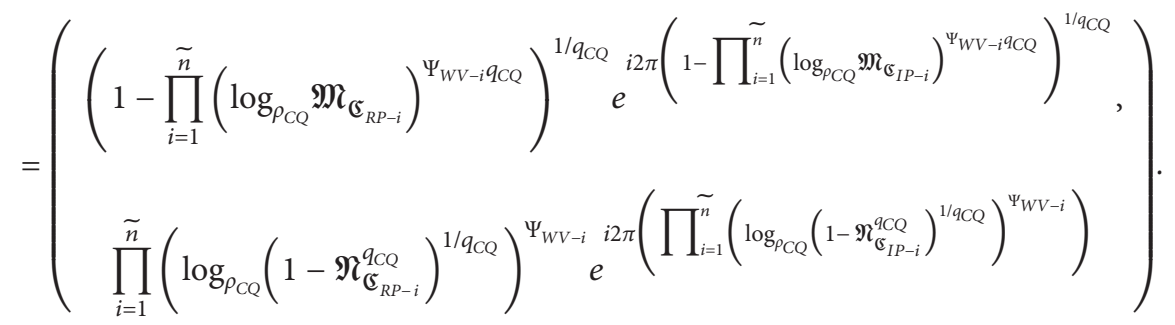

Property 1. For any family of CQROFNs $\operatorname{LCQROFWA}\left(\mathfrak{夭}_{\mathrm{CQ}-1}, \mathfrak{夭}_{\mathrm{CQ}-2}, \ldots, \mathfrak{夭}_{\mathrm{CQ}-\tilde{n}}\right)=\log _{\rho_{\mathrm{CQ}}} \mathfrak{\mathfrak { c }}_{\mathrm{CQ}}$ $\mathfrak{夭}_{C Q}=\mathfrak{夭}_{C Q-i}, i=1,2, \ldots, \widetilde{n}$, we have 
Complexity

15

Proof. We know that $\mathfrak{G}_{\mathrm{CQ}-i}=\left(\mathfrak{M}_{\mathfrak{C}_{R P-i}} e^{i 2 \pi\left(\mathfrak{M}_{\mathbb{G}_{I P-i}}\right)}\right.$, $\mathfrak{N}_{\mathbb{C}_{R P-i}} e^{i 2 \pi\left(\mathfrak{\Re}_{\mathbb{G}_{I P-i}}\right)}$ with $\mathfrak{C}_{\mathrm{CQ}}=\mathfrak{C}_{\mathrm{CQ}-i}, i=1,2, . ., \bar{n}$, then

$$
\begin{aligned}
& \operatorname{LCQROFWA}\left(\mathfrak{夭}_{\mathrm{CQ-1}}, \mathfrak{夭}_{\mathrm{CQ}-2}, \ldots, \mathfrak{夭}_{\mathrm{CQ}-\tilde{n}}\right)
\end{aligned}
$$

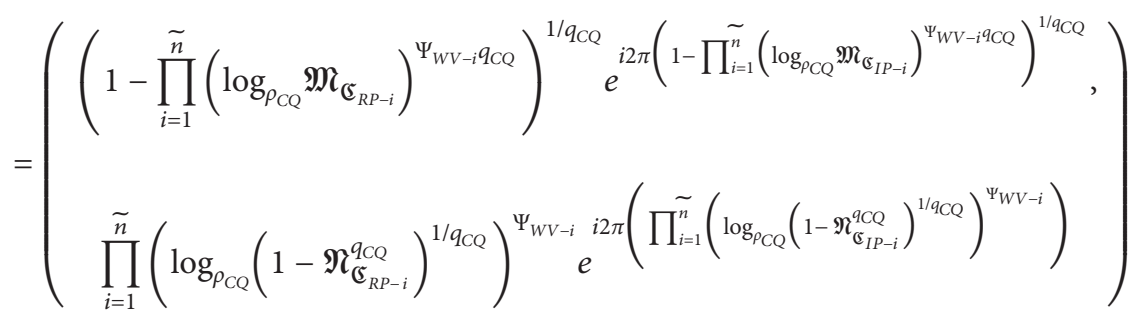

$$
\begin{aligned}
& =\left(\begin{array}{c}
\left(1-\prod_{i=1}^{\tilde{n}}\left(\log _{\rho_{C Q}} \mathfrak{M}_{\mathbb{C}_{R P}}\right)^{\Psi_{W V-i} q_{C Q}}\right)^{1 / q_{C Q}} e^{i 2 \pi\left(1-\prod_{i=1}^{\tilde{n}}\left(\log _{\rho C Q} \mathfrak{M}_{\mathbb{G}_{I P}}\right)^{\Psi_{W V-i} q_{C Q}}\right)^{1 / q C Q}}, \\
\prod_{i=1}^{\tilde{n}}\left(\log _{\rho_{C Q}}\left(1-\mathfrak{M}_{\mathbb{C}_{R P}}^{q_{C Q}}\right)^{1 / q_{C Q}}\right)^{\Psi_{W V-i}} e^{i 2 \pi\left(\prod_{i=1}^{\tilde{n}}\left(\log _{\rho_{C Q}}\left(1-\mathfrak{M}_{\mathbb{S}_{I P}}^{q_{C Q}}\right)^{1 / q q_{C Q}}\right)^{\Psi_{W V-i}}\right)}
\end{array}\right)
\end{aligned}
$$

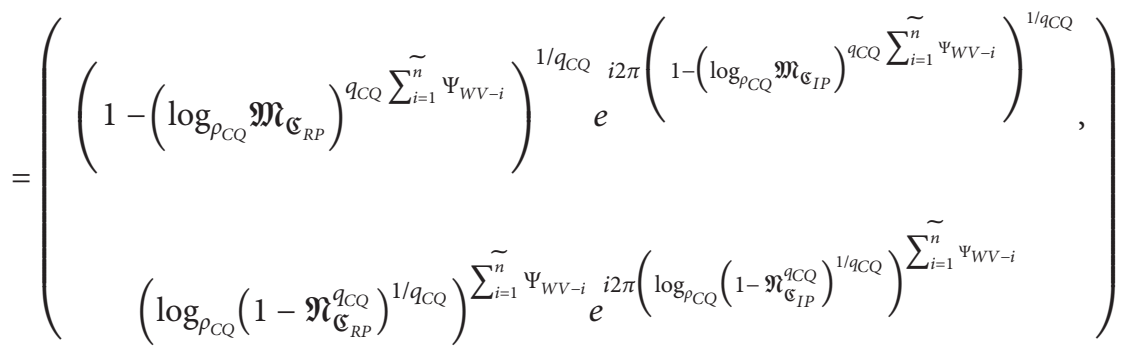

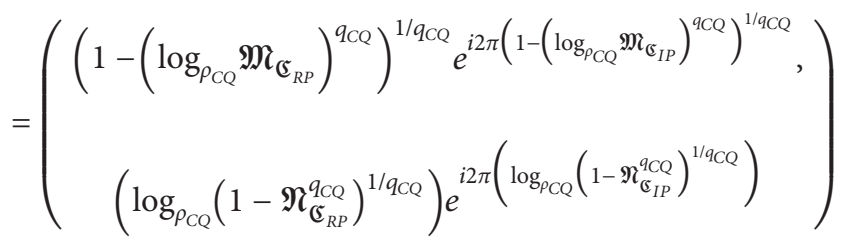

$$
\begin{aligned}
& =\log _{\rho_{C Q}} \mathfrak{夭}_{C Q} \text {. }
\end{aligned}
$$

Property 2. For any family of CQROFNs $\mathfrak{S}_{\mathrm{CQ}-i}=\left(\mathfrak{M}_{\mathfrak{G}_{R P-i}} e^{i 2 \pi\left(\mathfrak{M}_{\mathfrak{G}_{I P-i}}\right)}, \mathfrak{N}_{\mathbb{E}_{R P-i}} e^{i 2 \pi\left(\mathfrak{M}_{\mathfrak{G}_{I P-i}}\right)}\right), i=1,2, \ldots, \widetilde{n}$, with $\mathfrak{C}_{C Q}^{-}=\left(\min _{i}\left\{\mathfrak{M}_{\mathfrak{C}_{R P-i}}\right\} e^{i 2 \pi\left(\min _{i}\right.}\left\{\mathfrak{M}_{\mathbb{C}_{I P-i}}\right\}\right), \min _{i}\left\{\mathfrak{M}_{\mathfrak{C}_{R P-i}}\right\}$ $e^{i 2 \pi\left(\min _{i}\left\{\mathfrak{\Re}_{\mathbb{G}_{I P-i}}\right\}\right)} \quad$ and $\quad \mathfrak{E}_{C Q}^{+}=\left(\max _{i}\left\{\mathfrak{M}_{\mathfrak{E}_{R P-i}}\right\} e^{i 2 \pi\left(\max _{i}\right.}\right.$

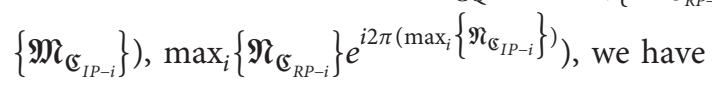


16

Complexity

$$
\begin{aligned}
\log _{\rho_{\mathrm{CQ}}} \mathfrak{夭}_{C Q}^{-} & \leq \mathrm{LCQROFWA}\left(\mathfrak{夭}_{\mathrm{CQ}-1}, \mathfrak{夭}_{\mathrm{CQ}-2}, \ldots, \mathfrak{夭}_{\mathrm{CQ}-\tilde{n}}\right) \\
& \leq \log _{\rho_{\mathrm{CQ}}} \mathfrak{夭}_{\mathrm{CQ}}^{+} .
\end{aligned}
$$

Proof. It is clear that $\min _{i}\left\{\mathfrak{M}_{\mathbb{E}_{R P-i}}\right\} \leq \mathfrak{M}_{\mathbb{C}_{R P-i}} \leq$ $\max _{i}\left\{\mathfrak{M}_{\mathfrak{E}_{R P-i}}\right\}, \min _{i}\left\{\mathfrak{M}_{\mathbb{E}_{I P-i}}\right\} \leq \mathfrak{M}_{\mathfrak{E}_{I P-i}} \leq \max _{i}\left\{\mathfrak{M}_{\mathfrak{C}_{I P-i}}\right\}, \min _{i}$ $\left\{\mathfrak{N}_{\mathfrak{C}_{R P-i}}\right\} \leq \mathfrak{N}_{\mathfrak{C}_{R P-i}} \leq \max _{i}\left\{\mathfrak{N}_{\mathfrak{C}_{R P-i}}\right\}$, and $\min _{i}\left\{\mathfrak{N}_{\mathfrak{C}_{I P-i}}\right\} \leq$ $\mathfrak{N}_{\mathfrak{C}_{I P-i}} \leq \max _{i}\left\{\mathfrak{N}_{\mathfrak{C}_{I P-i}}\right\}$; then, $\mathfrak{E}_{C Q}^{-} \leq \mathfrak{E}_{C Q} \leq \mathfrak{E}_{C Q}^{+}$. Consider that

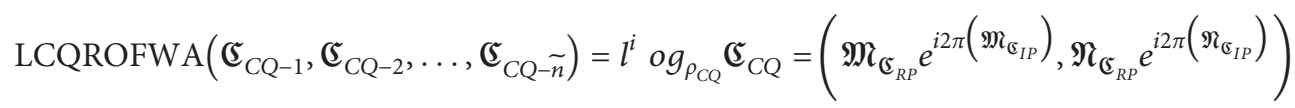

$$
\begin{aligned}
& l^{i} \operatorname{g}_{\rho_{C Q}} \mathfrak{S}_{C Q}^{-}=\left(\mathfrak{M}_{\mathbb{C}_{R P}^{-}} e^{i 2 \pi\left(\mathfrak{M}_{\mathbb{G}_{I P}^{-}}\right)}, \mathfrak{N}_{\mathbb{C}_{R P}^{-}} e^{i 2 \pi\left(\mathfrak{M}_{\mathbb{E}_{I P}^{-}}\right)}\right) \\
& l^{i} \operatorname{gog}_{\rho_{C Q}} \mathfrak{E}_{C Q}^{+}=\left(\mathfrak{M}_{\mathbb{E}_{R P}^{+}} e^{i 2 \pi\left(\mathfrak{M}_{\mathbb{E}_{I P}^{+}}\right)}, \mathfrak{N}_{\mathbb{E}_{R P}^{+}} e^{i 2 \pi\left(\mathfrak{\Re}_{\mathbb{C}_{I P}^{+}}\right)}\right) .
\end{aligned}
$$

By using monotonicity of logarithmic function, we obtain

$$
\begin{aligned}
& \mathfrak{M}_{\mathfrak{C}_{R P}} e^{i 2 \pi\left(\mathfrak{M}_{\mathbb{E}_{I P}}\right)}=\left(1-\prod_{i=1}^{\tilde{n}}\left(\log _{\rho_{C Q}} \mathfrak{M}_{\mathfrak{G}_{R P-i}}\right)^{\Psi_{W V-i} q_{C Q}}\right)^{1 / q_{C Q}} e^{i 2 \pi\left(1-\prod_{i=1} \tilde{n}\left(\log _{\rho C Q} \mathfrak{M}_{\mathbb{G}_{I P-i}}\right)^{\Psi_{W V-i} q_{C Q}}\right)^{1 / 9 C Q}}
\end{aligned}
$$

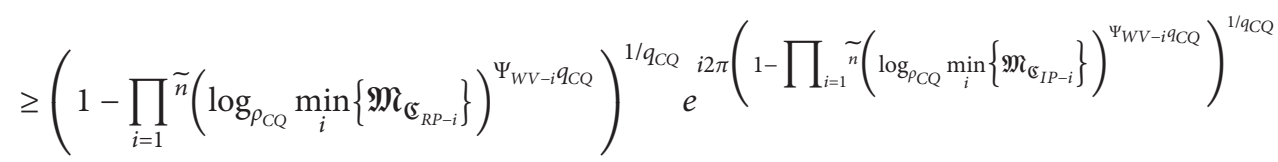

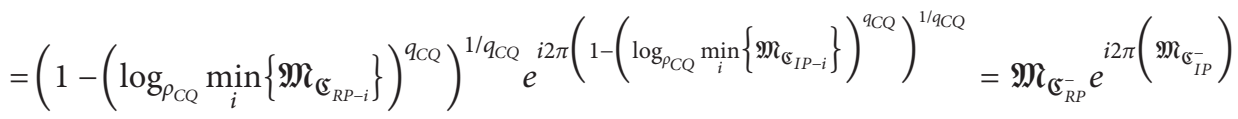

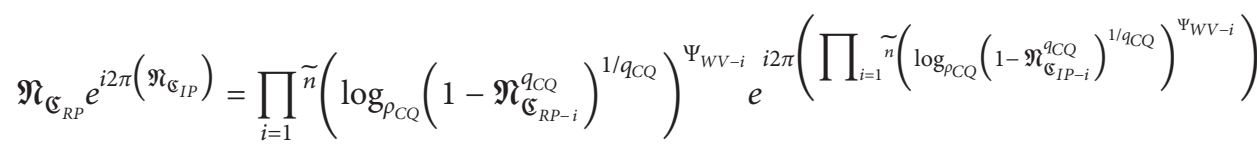

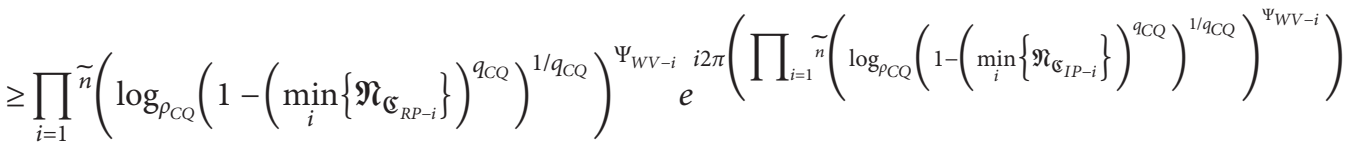

$$
\begin{aligned}
& =\prod_{i=1}^{\tilde{n}}\left(\log _{\rho_{C Q}}\left(1-\left(\min _{i}\left\{\mathfrak{N}_{\mathbb{C}_{R P-i}}\right\}\right)^{q_{C Q}}\right)^{1 / q_{C Q}}\right) e^{i 2 \pi}\left(\prod_{i=1}^{\tilde{n}}\left(\log _{\rho_{C Q}}\left(1-\left(\min _{i}\left\{\mathfrak{\Re}_{\mathbb{E}_{I P-i}}\right\}\right)^{q C Q}\right)^{1 / q_{C Q}}\right)\right) \\
& =\mathfrak{N}_{\mathbb{C}_{R P}^{-}} e^{i 2 \pi\left(\mathfrak{\Re}_{\mathfrak{G}_{I P}^{-}}\right)},
\end{aligned}
$$


and also

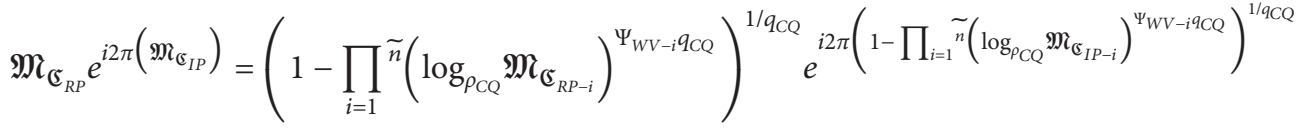

$$
\begin{aligned}
& \leq\left(1-\prod_{i=1}^{\tilde{n}}\left(\log _{\rho_{C Q}} \max _{i}\left\{\mathfrak{M}_{\mathfrak{C}_{R P-i}}\right\}\right)^{\Psi_{W V-i} q_{C Q}}\right)^{1 / q_{C Q}} e^{i 2 \pi\left(1-\prod_{i=1}^{\tilde{n}}\left(\log _{\rho C Q} \max _{i}\left\{\mathfrak{M}_{\mathfrak{G}_{I P-i}}\right\}\right)^{\Psi_{W V-i} q_{C Q}}\right)^{1 / q C Q}}
\end{aligned}
$$

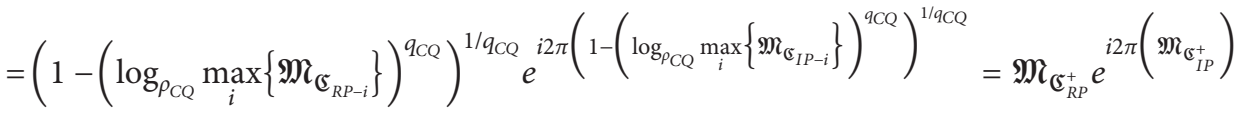

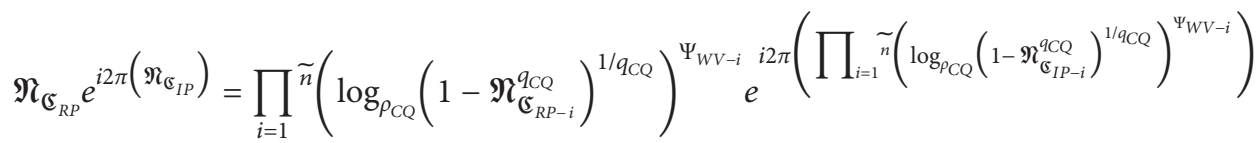

$$
\begin{aligned}
& \leq \prod_{i=1}^{\tilde{n}}\left(\log _{\rho_{C Q}}\left(1-\left(\max _{i}\left\{\mathfrak{N}_{\mathfrak{C}_{R P-i}}\right)^{q_{C Q}}\right)^{1 / q_{C Q}}\right)^{\Psi_{W V-i}} e^{i 2 \pi(}\left(\prod_{i=1}^{\tilde{n}}\left(\log _{\rho_{C Q}}\left(1-\left(\max _{i}\left\{\mathfrak{\Re}_{\mathbb{E}_{I P-i}}\right\}\right)^{q C Q}\right)^{1 / 9 C Q}\right)^{\Psi_{W V-i}}\right)\right.
\end{aligned}
$$

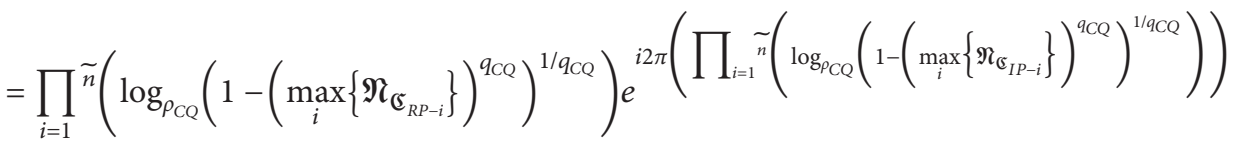

$$
\begin{aligned}
& =\mathfrak{N}_{\mathbb{E}_{R P}^{+}} e^{i 2 \pi\left(\mathfrak{N}_{\mathbb{E}_{I P}^{+}}\right)} \text {. }
\end{aligned}
$$

By using equation (3), we obtain

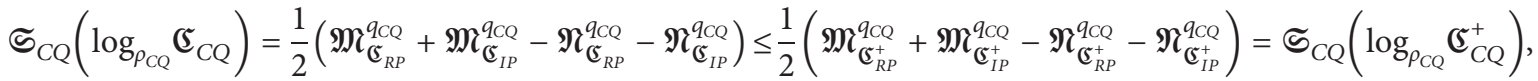

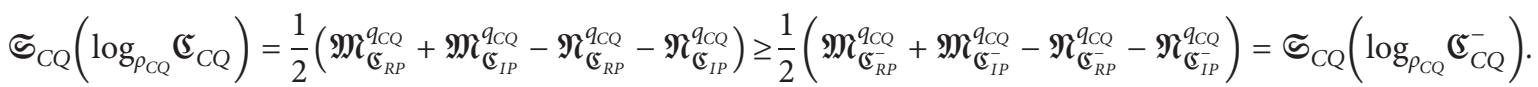

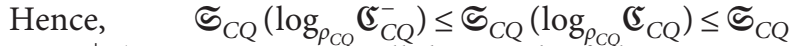
$\left(\log _{\rho_{C Q}} \mathfrak{C}_{C Q}^{+}\right)$. Moreover, we will discuss the following cases for interrelationships among the CQROFSs:

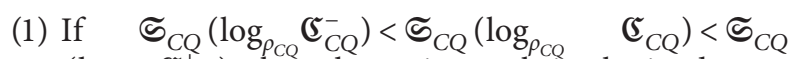
$\left(\log _{\rho_{C Q}}\left(\mathfrak{E}_{C Q}^{+}\right)\right.$, then the main result is obtained

(2) If $\mathfrak{S}_{C Q}\left(\log _{\rho_{C Q}} \mathfrak{夭}_{C Q}^{+}\right)=\mathfrak{S}_{C Q}\left(\log _{\rho_{C Q}}\left(\mathfrak{S}_{C Q}\right)\right.$, then $(1 / 2)$ $\left(\mathfrak{M}_{\mathfrak{C}_{R P}}^{q_{C Q}}+\mathfrak{M}_{\mathfrak{C}_{I P}}^{C_{I P}}-\mathfrak{N}_{\mathfrak{G}_{R P}}^{q_{C Q}}-\mathfrak{N}_{\mathfrak{G}_{I P}}^{q_{C Q}}\right)=(1 / 2) \quad\left(\mathfrak{M}_{\mathfrak{C}_{R P}^{+}}^{q_{C Q}}+\right.$ $\left.\mathfrak{M}_{\mathfrak{C}_{I P}^{+}}^{q_{C Q}}-\mathfrak{N}_{\mathfrak{C}_{R P}^{+}}^{q_{C Q}}-\mathfrak{N}_{\mathfrak{C}_{I P}^{+}}^{q_{C Q}}\right) \quad \Longrightarrow \mathfrak{M}_{\mathfrak{C}_{R P}}=\mathfrak{M}_{\mathfrak{C}_{R P}^{+}}, \mathfrak{M}_{\mathfrak{C}_{I P}}=$ $\mathfrak{M}_{\mathfrak{G}_{I P}^{+}}$and $\mathfrak{N}_{\mathfrak{C}_{R P}}=\mathfrak{N}_{\mathfrak{C}_{R P}^{+}}, \mathfrak{N}_{\mathfrak{C}_{I P}}=\mathfrak{N}_{\mathfrak{G}_{I P}^{+}}$, such that $\mathfrak{H}_{C Q}\left(\log _{\rho_{C Q}} \mathfrak{\mho}_{C Q}^{+}\right)=\mathfrak{H}_{C Q}\left(\log _{\rho_{C Q}}\left(\mathfrak{夭}_{C Q}\right)\right.$

(3) If $\mathfrak{S}_{C Q}\left(\log _{\rho_{C Q}} \mathfrak{⿰}_{C Q}^{-}\right)=\mathfrak{S}_{C Q}\left(\log _{\rho_{C Q}}\left(\mathfrak{S}_{C Q}\right)\right.$, then $(1 / 2)$ $\left(\mathfrak{M}_{\mathfrak{C}_{R P}}^{q_{C Q}}+\mathfrak{M}_{\mathfrak{C}_{I P}}^{C_{I P}}-\mathfrak{N}_{\mathfrak{C}_{R P}}^{q_{\mathrm{CQ}}}-\mathfrak{N}_{\mathfrak{G}_{I P}}^{q_{\mathrm{CQ}}}\right)=(1 / 2) \quad\left(\mathfrak{M}_{\mathfrak{C}_{R P}}^{q_{C Q}}+\right.$ $\left.\mathfrak{M}_{\mathfrak{C}^{-}}^{q_{C Q}}-\mathfrak{N}_{\mathfrak{C}_{R P}^{-}}^{q_{C Q}}-\mathfrak{N}_{\mathfrak{C}_{I P}^{-}}^{q_{C Q}}\right) \quad \Longrightarrow \mathfrak{M}_{\mathfrak{C}_{R P}}=\mathfrak{M}_{\mathfrak{G}_{R P}^{-}}, \mathfrak{M}_{\mathfrak{C}_{I P}}=$ $\mathfrak{M}_{\mathfrak{G}_{I P}^{-}}$and $\mathfrak{N}_{\mathfrak{C}_{R P}}=\mathfrak{N}_{\mathfrak{E}_{R P}^{-}}, \mathfrak{N}_{\mathfrak{G}_{I P}}=\mathfrak{N}_{\mathfrak{G}_{I P}^{-}}$, such that $\mathfrak{H}_{C Q}\left(\log _{\rho_{C Q}}\left(\mathfrak{r}_{C Q}^{-}\right)=\mathfrak{H}_{C Q}\left(\log _{\rho_{C Q}} \mathfrak{E}_{C Q}\right)\right.$
By using the above three cases, we obtain

$$
\begin{aligned}
\log _{\rho_{C Q}} \mathfrak{r}_{C Q}^{-} & \leq \operatorname{LCQROFWA}\left(\mathfrak{夭}_{C Q-1}, \mathfrak{夭}_{C Q-2}, \ldots, \mathfrak{c}_{C Q-\tilde{n}}\right) \\
& \leq \log _{\rho_{C Q}} \mathfrak{夭}_{C Q}^{+} .
\end{aligned}
$$

Property 3. For any family of CQROFNs $\mathfrak{夭}_{\mathrm{CQ}-i}=$ $\left(\mathfrak{M}_{\mathfrak{C}_{R P-i}} e^{i 2 \pi\left(\mathfrak{M}_{\mathbb{E}_{I P-i}}\right)}, \mathfrak{N}_{\mathfrak{C}_{R P-i}} e^{i 2 \pi\left(\mathfrak{M}_{\mathbb{E}_{I P-i}}\right)}\right), i=1,2, \ldots, \tilde{n}, \quad$ and $\mathfrak{S}_{\mathrm{CQ}-i}^{*}=\left(\mathfrak{M}_{\mathbb{C}_{R P-i}^{*}} e^{i 2 \pi\left(\mathfrak{M}_{\mathbb{E}_{I P-i}^{*}}^{*}\right)}, \mathfrak{N}_{\mathbb{C}_{R P-i}^{*}} e^{i 2 \pi\left(\mathfrak{\Re}_{\mathbb{E}_{I P-i}^{*}}\right)}\right)$, if $\quad \mathfrak{S}_{\mathrm{CQ}-i} \leq$ $\mathfrak{C}_{C Q-i}^{*}$, that is, $\mathfrak{M}_{\mathfrak{C}_{R P-i}} \leq \mathfrak{M}_{\mathfrak{C}_{R P-i}^{*}}^{*}, \mathfrak{M}_{\mathfrak{C}_{I P-i}} \leq \mathfrak{M}_{\mathfrak{C}_{I P-i}^{*}}, \mathfrak{N}_{\mathfrak{C}_{R P-i}} \geq$ $\mathfrak{N}_{\mathfrak{C}_{R P-i}^{*}}, \mathfrak{N}_{\mathfrak{C}_{I P-i}} \geq \mathfrak{N}_{\mathfrak{C}_{I P-i}^{*}}$, we have

$$
\begin{aligned}
& \operatorname{LCQROFWA}\left(\mathfrak{夭}_{\mathrm{CQ}-1}, \mathfrak{夭}_{\mathrm{CQ}-2}, \ldots, \mathfrak{夭}_{\mathrm{CQ}-\tilde{n}}\right) \\
& \leq \mathrm{LCQROFWA}\left(\mathfrak{夭}_{\mathrm{CQ}-1}^{*}, \mathfrak{夭}_{\mathrm{CQ}-2}^{*}, \ldots, \mathfrak{夭}_{\mathrm{CQ}-\tilde{n}}^{*}\right) \text {. }
\end{aligned}
$$


18

Complexity

Proof. Omitted.

Definition 7. For any family of CQROFNs $\mathfrak{C}_{C Q-i}=\left(\mathfrak{M}_{\mathfrak{C}_{R P-i}} e^{i 2 \pi\left(\mathfrak{M}_{\mathbb{C}_{I P-i}}\right)}, \mathfrak{N}_{\mathfrak{C}_{R P-i}} e^{i 2 \pi\left(\mathfrak{N}_{\mathbb{C}_{I P-i}}\right)}\right), i=1,2, \ldots, \widetilde{n}$, if $0<\rho_{C Q} \leq \min _{i}\left\{\mathfrak{M}_{\mathfrak{C}_{R P-i}},\left(1-\mathfrak{N}_{\mathfrak{C}_{R P-i}}^{q_{C Q}}\right)^{1 / q_{C Q}}\right\} \leq 1, \quad 0<\rho_{C Q} \leq$ $\min _{i}\left\{\mathfrak{M}_{\mathfrak{C}_{I P-i}},\left(1-\mathfrak{N}_{\mathfrak{C}_{I P-i}}^{q_{C Q}}\right)^{1 / q_{C Q}}\right\} \leq 1, \quad$ and $\quad \rho_{C Q} \neq 1, \quad$ the LCQROFOWA operators are demonstrated by LCQROFOWA: $\Xi^{n} \longrightarrow \Xi$, by

$$
\begin{aligned}
& \operatorname{LCQROFOWA}\left(\mathfrak{c}_{C Q-1}, \mathfrak{c}_{C Q-2}, \ldots, \mathfrak{c}_{C Q-\tilde{n}}\right) \\
& =\Psi_{W V-1} \log _{\rho_{C Q-\mathcal{O}(1)}} \mathfrak{S}_{C Q-\mathcal{O}(1)} \oplus \Psi_{W V-2} \log _{\rho_{\mathrm{CQ}-\mathcal{O}(2)}} \mathfrak{夭}_{C Q-\mathcal{O}(2)} \oplus \cdots \oplus \Psi_{W V-\widetilde{n}} \log _{\rho_{\mathrm{CQ}-\mathcal{O}(\tilde{n})}} \mathfrak{S}_{C Q-\mathcal{O}(\widetilde{n})} \text {, }
\end{aligned}
$$

where LCQROFOWA is a logarithmic CQROFOWA opaerator with weight vector $\Psi_{W V}=\left(\Psi_{W V-1}\right.$, $\left.\Psi_{W V-2}, \ldots, \Psi_{W V-\tilde{n}}\right)^{T}$ and $\sum_{i=1}^{\tilde{n}} \Psi_{W V-i}=1$. The term $\mathcal{O}$ expressed permutations with $\mathfrak{S}_{C Q-\mathcal{O}(i-1)} \geq \mathfrak{C}_{C Q-\mathcal{O}(i)}, i=1,2, \ldots, \widetilde{n}$.
Theorem 9. For any family of CQROFNs $\mathfrak{S}_{C Q-i}=\left(\mathfrak{M}_{\mathfrak{C}_{R P-i}} e^{i 2 \pi\left(\mathfrak{M}_{\mathfrak{C}_{I P-i}}\right)}, \mathfrak{N}_{\mathfrak{C}_{R P-i}} e^{i 2 \pi\left(\mathfrak{N}_{\mathbb{C}_{I P-i}}\right)}\right), i=1,2, \ldots, \widetilde{n}$, and by using equation (40), we obtain

$\operatorname{LCQROFWA}\left(\mathfrak{夭}_{\mathrm{CQ-1}}, \mathfrak{c}_{\mathrm{CQ}-2}, \ldots, \mathfrak{c}_{\mathrm{CQ}-\tilde{n}}\right)$

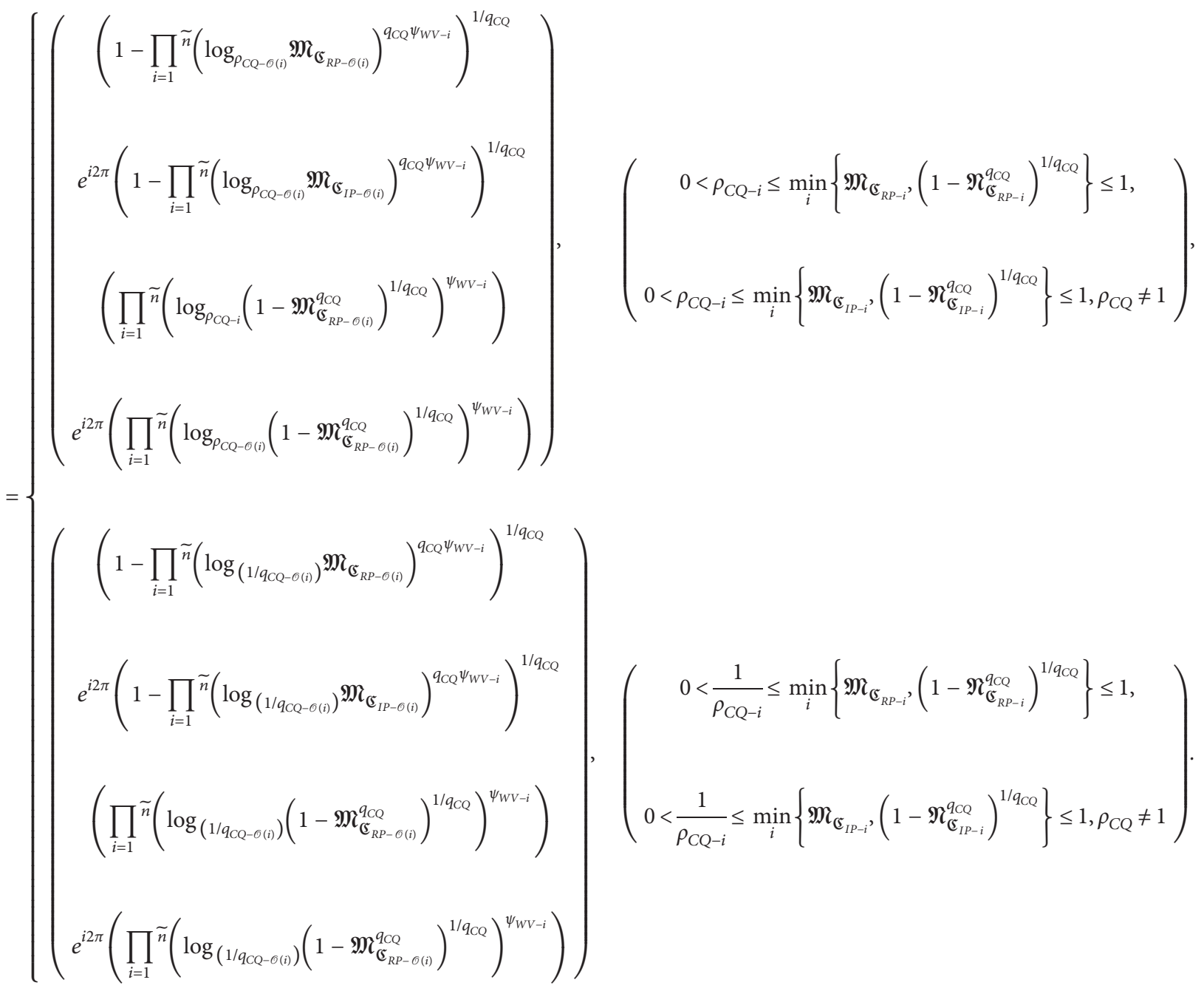


Complexity

19

Proof. Omitted (proof is similar to Theorem 8).

Definition 8. For any family of CQROFNs $\mathfrak{C}_{C Q-i}=\left(\mathfrak{M}_{\mathfrak{C}_{R P-i}} e^{i 2 \pi\left(\mathfrak{M}_{\mathbb{C}_{I P-i}}\right)}, \mathfrak{N}_{\mathfrak{C}_{R P-i}} e^{i 2 \pi\left(\mathfrak{N}_{\mathbb{C}_{I P-i}}\right)}\right), i=1,2, \ldots, \widetilde{n}$, if $0<\rho_{C Q} \leq \min _{i}\left\{\mathfrak{M}_{\mathfrak{C}_{R P-i}},\left(1-\mathfrak{N}_{\mathfrak{C}_{R P-i}}^{q_{C Q}}\right)^{1 / q_{C Q}}\right\} \leq 1, \quad 0<\rho_{C Q} \leq$ $\min _{i}\left\{\mathfrak{M}_{\mathfrak{C}_{I P-i}},\left(1-\mathfrak{N}_{\mathfrak{C}_{I P-i}}^{q_{C Q}}\right)^{1 / q_{C Q}}\right\} \leq 1, \quad$ and $\quad \rho_{C Q} \neq 1$, the LCQROFWG operators are demonstrated by LCQROFWG: $\Xi^{\tilde{n}} \longrightarrow \Xi$, by

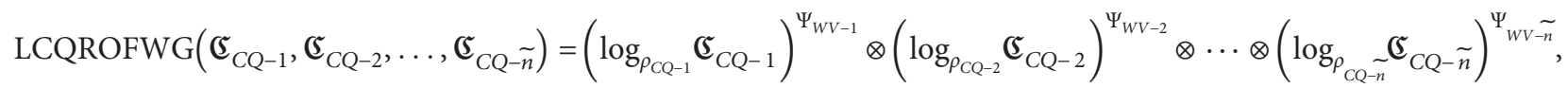

where LCQROFWG is a logarithmic CQROFWG operator with weight vector $\Psi_{W V}=\left(\Psi_{W V-1}, \Psi_{W V-2}, \ldots, \Psi_{W V-\tilde{n}}\right)^{T}$ and $\sum_{i=1}^{n} \Psi_{W V-i}=1$.

$\operatorname{LCQROFWA}\left(\mathfrak{c}_{C Q-1}, \mathfrak{c}_{C Q-2}, \ldots, \mathfrak{c}_{\mathrm{CQ}-\tilde{n}}\right)$

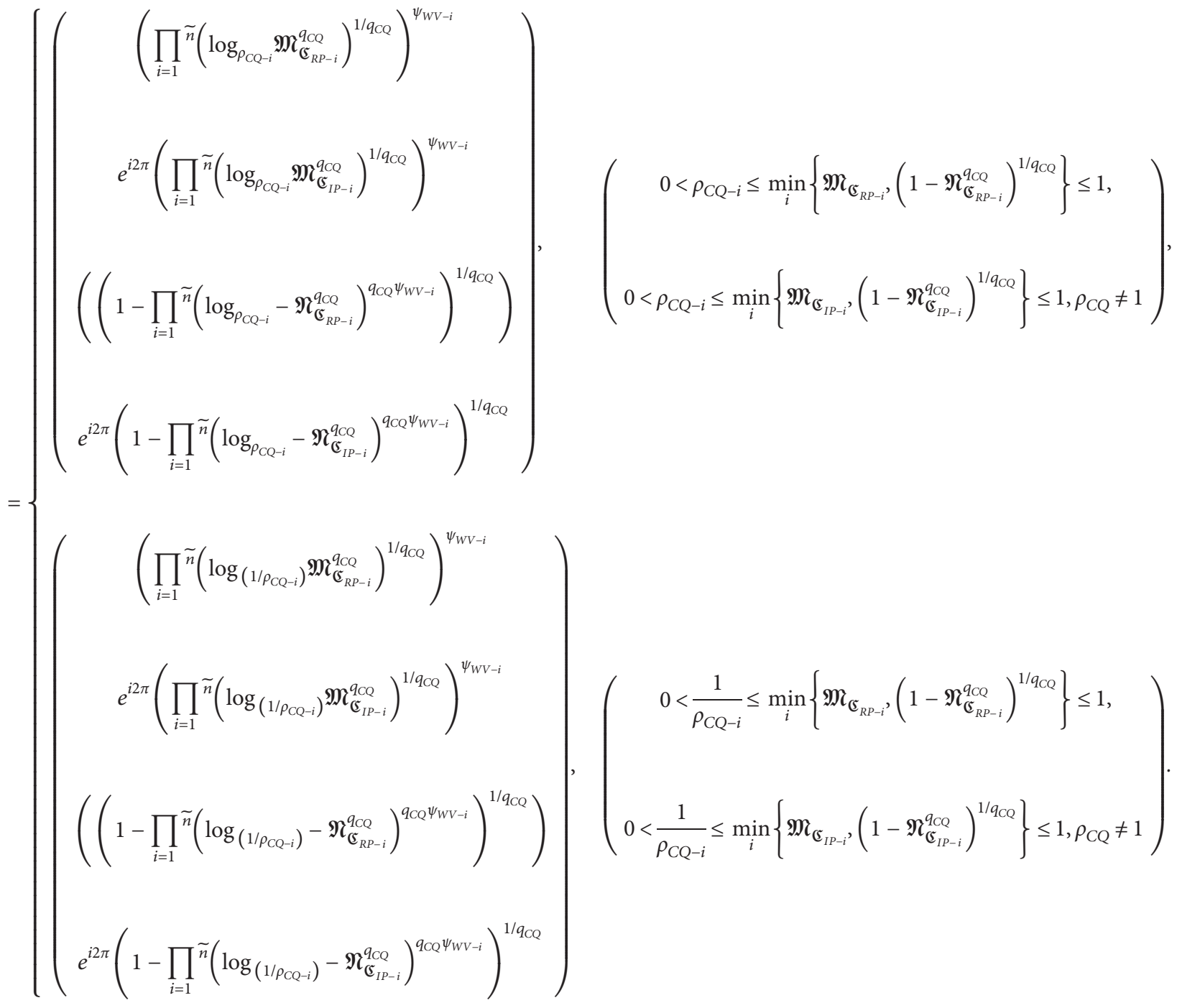


Proof. Omitted (proof is similar to Theorem 10).

Definition 9. For any family of CQROFNs $\mathfrak{C}_{C Q-i}=\left(\mathfrak{M}_{\mathfrak{C}_{R P-i}} e^{i 2 \pi\left(\mathfrak{M}_{\mathfrak{C}_{I P-i}}\right)}, \mathfrak{N}_{\mathfrak{C}_{R P-i}} e^{i 2 \pi\left(\mathfrak{\Re}_{\mathbb{C}_{I P-i}}\right)}\right), i=1,2, \ldots, \widetilde{n}$, if $0<\rho_{C Q} \leq \min _{i}\left\{\mathfrak{M}_{\mathfrak{C}_{R P-i}},\left(1-\mathfrak{N}_{\mathfrak{C}_{R P-i}}^{q_{C Q}}\right)^{1 / q_{C Q}}\right\} \leq 1, \quad 0<\rho_{C Q} \leq$ $\min _{i}\left\{\mathfrak{M}_{\mathfrak{C}_{I P-i}},\left(1-\mathfrak{N}_{\mathfrak{夭}_{I P-i}}^{q_{C Q}}\right)^{1 / q_{C Q}}\right\} \leq 1, \quad$ and $\quad \rho_{C Q} \neq 1, \quad$ the LCQROFOWG operators are demonstrated by LCQROFOWG: $\Xi^{\tilde{n}} \longrightarrow \Xi$, by

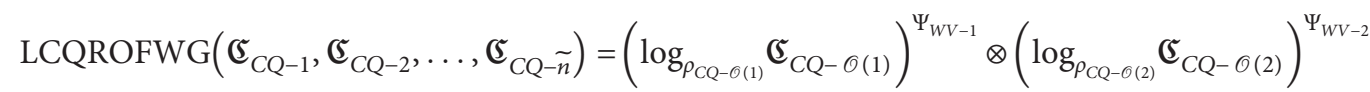

$$
\begin{aligned}
& \otimes \cdots \otimes\left(\log _{\rho_{C Q-\mathcal{O}(n)}} \mathfrak{S}_{C Q-\mathcal{O}(\tilde{n})}\right)^{\Psi}
\end{aligned}
$$

where LCQROFOWG is a logarithmic CQROFOWG operator with weight v vector $\Psi_{W V}=\left(\Psi_{W V-1}, \Psi_{W V-2}, \ldots, \Psi_{W V-\tilde{n}}\right)^{T}$ and $\sum_{i=1}^{\tilde{n}} \Psi_{W V-i}=1$. The term 0 expressed permutations with $\mathfrak{夭}_{\mathrm{CQ}-\mathcal{O}(i-1)} \geq \mathfrak{夭}_{\mathrm{CQ}-\mathcal{O}(i)}, i=1,2, \ldots, \tilde{n}$.

$\operatorname{LCQROFWA}\left(\mathfrak{c}_{\mathrm{CQ-1}}, \mathfrak{夭}_{\mathrm{CQ}-2}, \ldots, \mathfrak{c}_{\mathrm{CQ}-\tilde{n}}\right)$

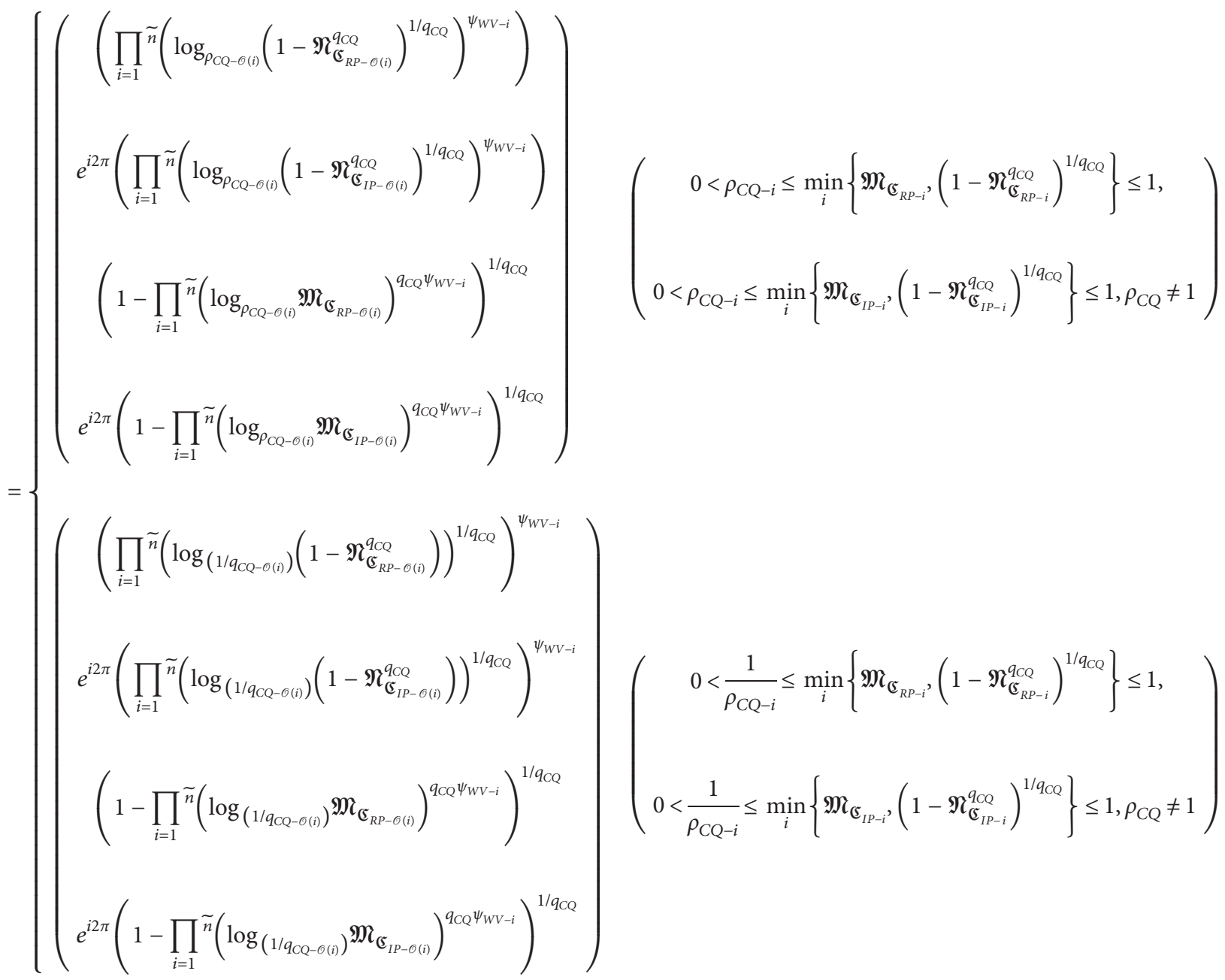


Proof. Omitted. (Proof is similar to Theorem 10).

$$
\begin{aligned}
& \text { Remark 2. When } \rho_{C Q-1}=\rho_{C Q-2}=\cdots=\rho_{C Q-\tilde{n}}=\rho_{C Q}, \\
& 0<\rho_{C Q-i} \leq \min _{i}\left\{\mathfrak{M}_{\mathfrak{C}_{R P-i}},\left(1-\mathfrak{N}_{\mathfrak{C}_{R P-i}}^{q_{C Q}}\right)^{1 / q_{C Q}}\right\} \leq 1, \\
& 0<\rho_{C Q-i} \leq \min _{i}\left\{\mathfrak{M}_{\mathfrak{C}_{I P-i}},\left(1-\mathfrak{N}_{\mathfrak{C}_{I P-i}}^{q_{C Q}}\right)^{1 / q_{C Q}}\right\} \leq 1, \rho_{C Q} \neq 1 \text {, then }
\end{aligned}
$$

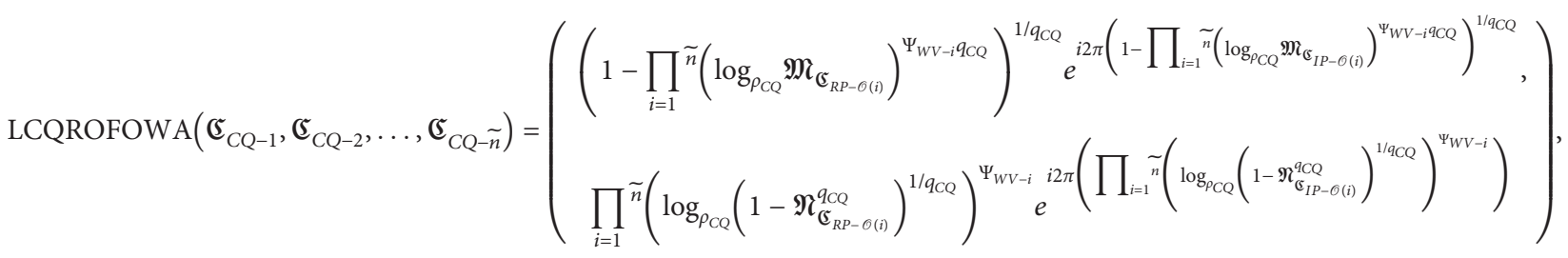

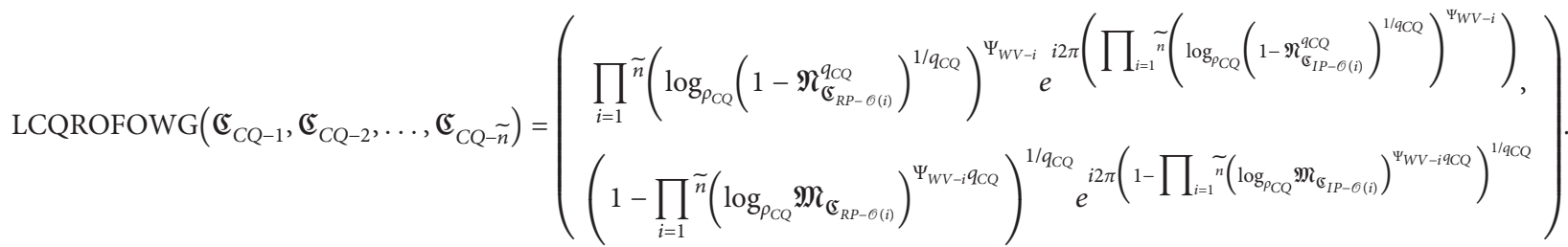

\section{MADM Algorithm Based on LCQROFWA and LCQROFWG}

In this section, we address the MADM algorithm to solve the decision-making problem under the CQROFS environment followed by the illustrative examples and the comparative analysis.

5.1. Proposed MADM Algorithm. For a MADM problem, consider a family of alternatives such that $\mathfrak{c}_{C Q}=\left\{\mathfrak{c}_{C Q-1}, \mathfrak{c}_{C Q-2}, \ldots, \mathfrak{c}_{C Q-\tilde{n}}\right\}$ and their attributes $\mathscr{C}_{C Q}=\left\{\mathscr{C}_{C Q-1}, \mathscr{C}_{C Q-2}, \ldots, \mathscr{C}_{C Q-\tilde{n}}\right\}$. Assume that the weight vector of the given attributes is denoted by $\Psi_{W V}=\left\{\Psi_{W V-1}, \Psi_{W V-2}, \ldots, \Psi_{W V-\tilde{n}}\right\}$ such that $\Psi_{W V-i}>0 ; \sum_{i=1}^{\tilde{n}} \Psi_{W V-i}=1$. To evaluate the given alternatives under the set of the attributes, an expert has been invited who can give the information of the alternatives in terms of the CQROFNs $\quad \mathfrak{S}_{C Q-i j}=\left(\mathfrak{M}_{\mathfrak{C}_{R P-i j}} e^{i 2 \pi\left(\mathfrak{M}_{\mathfrak{E}_{I P-i j}}\right)}, \mathfrak{N}_{\mathfrak{C}_{R P-i j}}\right.$ $e^{i 2 \pi\left(\Re_{\mathfrak{E}_{I P-i j}}\right)}$ such that sum of the q-powers of the real parts (also for imaginary parts) of the truth and falsity degrees cannot be exceeded by one, that is, $0 \leq \mathfrak{M}_{\mathfrak{C}_{R P}}^{q_{C Q}}\left(\sim_{x}^{x}\right)+\mathfrak{N}_{\mathfrak{C}_{R P}}^{q_{C Q}}$

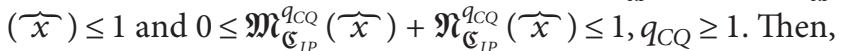
the main aim of the decision-making problem is to find the most suitable alternatives for the required task. To find them, we address an algorithm by utilizing the given CQROFN information of the expert and the proposed logarithm operators. The presented MADM approach has been summarized with the following steps:

Step 1: arrange the collective information of each alternative in terms of the decision matrix where each entry of the matrix is in the form of CQROFNs. This matrix is represented as

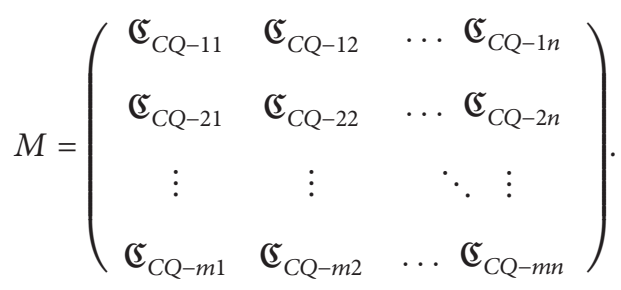

Step 2: taking the decision matrix $M$ and attribute weights $\Psi_{W V}=\left\{\Psi_{W V-1}, \Psi_{W V-2}, \ldots, \Psi_{W V-\tilde{n}}\right\}$, we aggregate the preferences of each alternative by utilizing either LCQROFWA or LCQROFWG operator. For example, take LCQROFWA operator to aggregate the rating of $\mathfrak{S}_{C Q-i j}=\left(\mathfrak{M}_{\mathfrak{C}_{R P-i j}} e^{i 2 \pi\left(\mathfrak{M}_{\mathfrak{C}_{I P-i j}}\right)}, \mathfrak{N}_{\mathfrak{C}_{R P-i j}}\right.$ $e^{i 2 \pi\left(\mathfrak{P}_{\mathbb{E}_{I P-i j}}\right)}$ for alternative $\mathfrak{S}_{C Q-i}$ and hence denote their overall value as $\mathfrak{E}_{C Q-i}=\left(R_{i} e^{i 2 \pi\left(I_{i}\right)}, N_{i} e^{i 2 \pi\left(P_{i}\right)}\right)$ where $R_{i}, I_{i}, N_{i}$, and $P_{i}$ are obtained from LCQROFWA operator provided $\rho_{\mathrm{CQ}-i}<1$ as follows:

$$
\begin{aligned}
R_{i} & =\left(1-\prod_{j=1}^{\tilde{n}}\left(\log _{\rho_{C Q-i}} \mathfrak{M}_{\mathfrak{C}_{R P-i j}}\right)^{q_{C Q} \Psi_{W V-j}}\right)^{1 / q_{C Q}}, \\
I_{i} & =\left(1-\prod_{j=1}^{\tilde{n}}\left(\log _{\rho_{C Q-i}} \mathfrak{M}_{\mathfrak{C}_{I P-i j}}\right)^{q_{C Q} \Psi_{W V-j}}\right)^{1 / q_{C Q}}, \\
N_{i} & =\prod_{j=1}^{\tilde{n}}\left(\log _{\rho_{\mathrm{CQ}-i}}\left(1-\mathfrak{N}_{\mathfrak{C}_{R P-i j}}^{q_{C Q}}\right) \frac{1}{q_{C Q}}\right)^{\Psi_{W V-j}}, \\
P_{i} & =\prod_{j=1}^{\tilde{n}}\left(\log _{\rho_{C Q-i}}\left(1-\mathfrak{N}_{\mathfrak{C}_{I P-i j}}^{q_{C Q}}\right)^{\frac{1}{q_{C Q}}}\right)^{\Psi_{W V-j}} .
\end{aligned}
$$


Step 3: for each alternative, compute the score values of the obtained aggregated CQROFN $\mathfrak{c}_{\mathrm{CQ}-i}=\left(R_{i} e^{i 2 \pi\left(I_{i}\right)}\right.$, $\left.N_{i} e^{i 2 \pi\left(P_{i}\right)}\right)$ as

$$
\mathfrak{\Im}_{C Q}\left(\mathfrak{夭}_{C Q-i}\right)=\frac{R_{i}+I_{i}-N_{i}-P_{i}}{2} .
$$

If there is a tie between any two indices of the score values, then we compute the accuracy degree of such number by using the following equation:

$$
A_{C Q}\left(\mathfrak{S}_{C Q-i}\right)=\frac{R_{i}+I_{i}+N_{i}+P_{i}}{2} .
$$

Step 4: based on these score values, rank the given alternatives and hence select the most suitable one.

5.2. Illustrative Examples. To demonstrate the work of the above-stated algorithm, we examined some decision-making problems and compute their results by following the steps of the stated algorithm.

Example 1. Consider a decision-making issue, taken from Garg [14], about the choice of the ideal creation methodology. In it, an organization needs to make another item and is investigating the ideal objective to acquire the most elevated advantages. For this, in the wake of dissecting the market, they have thought about the five potential techniques after their starter screening and are characterized as follows:

(1) $\mathfrak{S}_{C Q-1}$ : producing an invention adapted to the powerful businesses

(2) $\mathfrak{C}_{C Q-2}$ : producing an invention adapted to the midlevel businesses

(3) $\mathfrak{c}_{C Q-3}$ : producing an invention adapted to the lowlevel businesses

(4) $\mathfrak{S}_{C Q-4}$ : producing an invention adapted to all businesses

(5) $\mathfrak{C}_{\mathrm{CQ-5}}$ : not producing any invention

To assess these, the organization thinks about the practical factor as the key for the following year. Based on these, they need to assess every methodology under the accompanying five general qualities:

(1) $\mathscr{C}_{\mathrm{CQ-1}}$ : profits in the powerful conditions

(2) $\mathscr{C}_{\mathrm{CQ-2}}$ : profits in the mid conditions

(3) $\mathscr{C}_{\mathrm{CQ}-3}$ : profits in the long conditions

(4) $\mathscr{C}_{\mathrm{CQ}-4}$ : danger of the manufacture plan

(5) $\mathscr{C}_{C Q-5}$ : other issues

To investigate the best object, we implemented the steps of the stated algorithm as follows :

Step 1: the assessment value of each alternative is given by an expert in the form of CQROFNs and their overall ratings values are summarized in Table 1.
Step 2: without loss of generality, we consider the logarithm index bases $\rho_{C Q}$ for each alternative under the different attribute in the form of matrix $D$, such that

$$
D=\left[\begin{array}{lllll}
0.1 & 0.1 & 0.1 & 0.1 & 0.1 \\
0.1 & 0.1 & 0.1 & 0.1 & 0.1 \\
0.1 & 0.1 & 0.1 & 0.1 & 0.1 \\
0.1 & 0.1 & 0.1 & 0.1 & 0.1 \\
0.1 & 0.1 & 0.1 & 0.1 & 0.1
\end{array}\right]
$$

By utilizing this matrix and the weight vector of the attributes as $(0.3,0.3,0.2,0.1,0.1)$, we aggregate the given information in Table 1 by using the stated LCQROFWA and LCQROFWG operators for $q_{S C}=8$. The results corresponding to each operator are listed in Table 2.

Step 3: we compute the score values of the obtained numbers (given in Table 2) and get the following:

By LCQROFWA operator,

$$
\begin{aligned}
& \mathfrak{S}_{C Q}\left(\mathfrak{S}_{C Q-1}\right)=0.5123, \\
& \mathfrak{S}_{C Q}\left(\mathfrak{S}_{C Q-2}\right)=0.499998, \\
& \mathfrak{S}_{C Q}\left(\mathfrak{S}_{C Q-3}\right)=0.512, \\
& \mathfrak{S}_{C Q}\left(\mathfrak{S}_{C Q-4}\right)=0.49998, \\
& \mathfrak{S}_{C Q}\left(\mathfrak{c}_{C Q-5}\right)=0.499986 .
\end{aligned}
$$

By LCQROFWG operator,

$$
\begin{aligned}
& \mathfrak{S}_{C Q}\left(\mathfrak{S}_{C Q-1}\right)=-0.5123, \\
& \mathfrak{S}_{C Q}\left(\mathfrak{S}_{C Q-2}\right)=-0.5121, \\
& \mathfrak{S}_{C Q}\left(\mathfrak{S}_{C Q-3}\right)=-0.4991, \\
& \mathfrak{S}_{C Q}\left(\mathfrak{S}_{C Q-4}\right)=-0.512, \\
& \mathfrak{S}_{C Q}\left(\mathfrak{S}_{C Q-5}\right)=-0.49988 .
\end{aligned}
$$

Furthermore, the geometrical representation of these obtained score values is shown in Figure 1.

Step 4: arrange the score values of the given alternatives and we obtain the suitable ranking order corresponding to the value obtained through LCQROFWA operator as

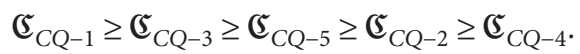

While by LCQROFWG operator, it is

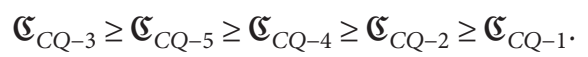

From these obtained ranking, we can analyze that the different operator (during the aggregation phase) gives a different best alternative. For instance, by taking LCQROFWA operator, we obtain $\mathfrak{E}_{\mathrm{CQ-1}}$ and through LCQROFWG operator, we get $\mathfrak{S}_{\mathrm{CQ}-3}$ alternative as the best one. This change in ranking occurs due to the structure of the stated operators. During the decision- 
TABLE 1: Rating of the given alternatives for Example 1.

\begin{tabular}{|c|c|c|c|c|c|}
\hline Alternatives/attributes & $\mathscr{C}_{C Q-1}$ & $\mathscr{C}_{C Q-2}$ & $\mathscr{C}_{C Q-3}$ & $\mathscr{C}_{C Q-4}$ & $\mathscr{C}_{C Q-5}$ \\
\hline$C_{C Q-1}$ & $\left(\begin{array}{c}0.9 e^{i 2 \pi(0.8)} \\
0.8 e^{i 2 \pi(0.7)}\end{array}\right)$ & $\left(\begin{array}{l}0.91 e^{i 2 \pi(0.81)}, \\
0.81 e^{i 2 \pi(0.71)}\end{array}\right)$ & $\left(\begin{array}{c}0.92 e^{i 2 \pi(0.82)} \\
0.82 e^{i 2 \pi(0.72)}\end{array}\right)$ & $\left(\begin{array}{c}0.93 e^{i 2 \pi(0.83)} \\
0.83 e^{i 2 \pi(0.73)}\end{array}\right)$ & $\left(\begin{array}{c}0.94 e^{i 2 \pi(0.84)} \\
0.84 e^{i 2 \pi(0.74)}\end{array}\right)$ \\
\hline$C_{C Q-2}$ & $\left(\begin{array}{l}0.8 e^{i 2 \pi(0.7)} \\
0.6 e^{i 2 \pi(0.7)}\end{array}\right)$ & $\left(\begin{array}{l}0.81 e^{i 2 \pi(0.71)} \\
0.61 e^{i 2 \pi(0.71)}\end{array}\right)$ & $\left(\begin{array}{c}0.82 e^{i 2 \pi(0.72)}, \\
0.62 e^{i 2 \pi(0.72)}\end{array}\right)$ & $\left(\begin{array}{c}0.83 e^{i 2 \pi(0.73)} \\
0.63 e^{i 2 \pi(0.73)}\end{array}\right)$ & $\left(\begin{array}{l}0.84 e^{i 2 \pi(0.74)} \\
0.64 e^{i 2 \pi(0.74)}\end{array}\right)$ \\
\hline$C_{C Q-3}$ & $\left(\begin{array}{c}0.9 e^{i 2 \pi(0.9)} \\
0.5 e^{i 2 \pi(0.6)}\end{array}\right)$ & $\left(\begin{array}{l}0.91 e^{i 2 \pi(0.91)}, \\
0.51 e^{i 2 \pi(0.61)}\end{array}\right)$ & $\left(\begin{array}{c}0.92 e^{i 2 \pi(0.92)} \\
0.52 e^{i 2 \pi(0.62)}\end{array}\right)$ & $\left(\begin{array}{c}0.93 e^{i 2 \pi(0.93)} \\
0.53 e^{i 2 \pi(0.63)}\end{array}\right)$ & $\left(\begin{array}{c}0.94 e^{i 2 \pi(0.94)} \\
0.54 e^{i 2 \pi(0.64)}\end{array}\right)$ \\
\hline$C_{C Q-4}$ & $\left(\begin{array}{l}0.8 e^{i 2 \pi(0.8)} \\
0.7 e^{i 2 \pi(0.7)}\end{array}\right)$ & $\left(\begin{array}{l}0.81 e^{i 2 \pi(0.81)}, \\
0.71 e^{i 2 \pi(0.71)}\end{array}\right)$ & $\left(\begin{array}{c}0.82 e^{i 2 \pi(0.82)}, \\
0.72 e^{i 2 \pi(0.72)}\end{array}\right)$ & $\left(\begin{array}{l}0.83 e^{i 2 \pi(0.83)} \\
0.73 e^{i 2 \pi(0.73)}\end{array}\right)$ & $\left(\begin{array}{c}0.84 e^{i 2 \pi(0.84)} \\
0.74 e^{i 2 \pi(0.74)}\end{array}\right)$ \\
\hline$C_{C Q-5}$ & $\left(\begin{array}{l}0.8 e^{i 2 \pi(0.5)} \\
0.6 e^{i 2 \pi(0.4)}\end{array}\right)$ & $\left(\begin{array}{l}0.81 e^{i 2 \pi(0.51)} \\
0.61 e^{i 2 \pi(0.41)}\end{array}\right)$ & $\left(\begin{array}{c}0.82 e^{i 2 \pi(0.52)} \\
0.62 e^{i 2 \pi(0.42)}\end{array}\right)$ & $\left(\begin{array}{c}0.83 e^{i 2 \pi(0.53)} \\
0.63 e^{i 2 \pi(0.43)}\end{array}\right)$ & $\left(\begin{array}{l}0.84 e^{i 2 \pi(0.54)} \\
0.64 e^{i 2 \pi(0.44)}\end{array}\right)$ \\
\hline
\end{tabular}

TABLE 2: Aggregated values by using LCQROFWA and LCQROFWG operators.

\begin{tabular}{lcr}
\hline Alternatives & By LCQROFW A operator & By LCQROFWG operator \\
\hline$C_{C Q-1}$ & $\left(0.999 e^{i 2 \pi(0.999)}, 0.011 e^{i 2 \pi(0.003)}\right)$ & $\left(0.036 e^{i 2 \pi(0.011)}, 0.999 e^{i 2 \pi(0.999)}\right)$ \\
$C_{C Q-2}$ & $\left(0.999 e^{i 2 \pi(0.999)}, 0.0011 e^{i 2 \pi(0.003)}\right)$ & $\left(0.011 e^{i 2 \pi(0.003)}, 0.999 e^{i 2 \pi(0.999)}\right)$ \\
$C_{C Q-3}$ & $\left(0.999 e^{i 2 \pi(0.999)}, 0.0002 e^{i 2 \pi(0.0011)}\right)$ & $\left(0.036 e^{i 2 \pi(0.036)}, 0.999 e^{i 2 \pi(0.999)}\right)$ \\
$C_{C Q-4}$ & $\left(0.999 e^{i 2 \pi(0.999)}, 0.003 e^{i 2 \pi(0.003)}\right)$ & $\left(0.011 e^{i 2 \pi(0.011)}, 0.999 e^{i 2 \pi(0.999)}\right)$ \\
$C_{C Q-5}$ & $\left(0.999 e^{i 2 \pi(0.999)}, 0.0011 e^{i 2 \pi(0.00004)}\right)$ & $\left(0.011 e^{i 2 \pi(0.0002)}, 0.999 e^{i 2 \pi(0.999)}\right)$ \\
\hline
\end{tabular}

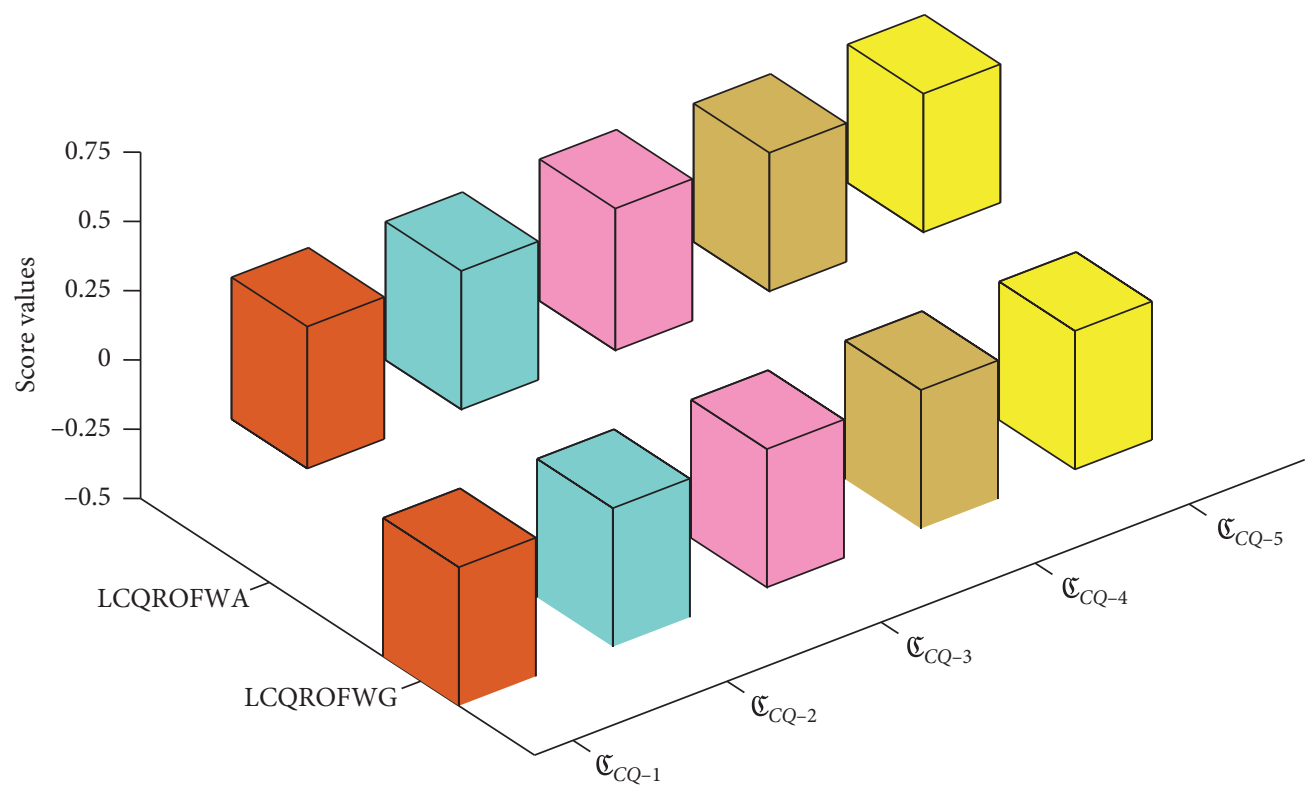

FIgURE 1: Geometrical representation of the score values for Example 1.

making phase, if an expert or decision-maker wants to optimize their decision based on the pessimism nature, then he/she can select the LCQROFWG operator to aggregate the information and hence choose the suitable alternative as $\mathfrak{c}_{\mathrm{CQ}-3}$. On the other hand, if a person wants to decide the alternative based on his/her optimism way, then select the $\mathfrak{c}_{C Q-1}$ alternative.
Example 2. Consider a MADM problem about the choice of the ideal creation methodology. In it, expect that an organization needs to make another item and they are investigating the ideal objective to acquire the most elevated advantages. For this, in the wake of dissecting the market, they have thought about the five potential techniques after their starter screening and are characterized as follows: 
(1) $\mathfrak{C}_{C Q-1}$ : producing an invention adapted to the powerful businesses

(2) $\mathfrak{C}_{C Q-2}$ : producing an invention adapted to the midlevel businesses

(3) $\mathfrak{c}_{C Q-3}$ : producing an invention adapted to the lowlevel businesses

(4) $\mathfrak{c}_{C Q-4}$ : producing an invention adapted to all businesses

(5) $\mathfrak{夭}_{C Q-5}$ : not producing any invention

To assess these, the organization thinks about the practical factor as the key for the following year. Based on these, they need to assess every methodology under the accompanying five general qualities:

(1) $\mathscr{C}_{C Q-1}$ : profits in the powerful conditions

(2) $\mathscr{C}_{C Q-2}:$ profits in the mid conditions

(3) $\mathscr{C}_{\mathrm{CQ}-3}$ : profits in the long conditions

(4) $\mathscr{C}_{C Q-4}$ : danger of the manufacture plan

(5) $\mathscr{C}_{C Q-5}$ : other issues

To investigate the best object, we implemented the steps of the stated algorithm as follows :

Step 1: an expert has been invited to evaluate the given alternative and they provide the rating in terms of the complex Pythagorean fuzzy numbers (CPFNs), which is a special case of the presented CQROFNs by taking $q_{S C}=2$. The rating of them is listed in Table 3 .

Step 2: we consider the logarithm index bases $\rho_{C Q}$ for each alternative as mentioned in the Matrix $D$ as

$$
D=\left[\begin{array}{lllll}
0.1 & 0.1 & 0.1 & 0.1 & 0.1 \\
0.1 & 0.1 & 0.1 & 0.1 & 0.1 \\
0.1 & 0.1 & 0.1 & 0.1 & 0.1 \\
0.1 & 0.1 & 0.1 & 0.1 & 0.1 \\
0.1 & 0.1 & 0.1 & 0.1 & 0.1
\end{array}\right],
$$

and hence implemented the LCQROFWA and LCQROFWG operators to aggregate the information by taking weight vector as $(0.3,0.3,0.2,0.1,0.1)$. The collective aggregated values corresponding to each operator are listed in Table 4.

Step 3: the score values corresponding to these aggregated numbers are calculated as follows:

By LCQROFWA operator,

$$
\begin{aligned}
& \mathfrak{S}_{C Q}\left(\mathfrak{S}_{C Q-1}\right)=0.495626, \\
& \mathfrak{S}_{C Q}\left(\mathfrak{S}_{C Q-2}\right)=0.481911, \\
& \mathfrak{S}_{C Q}\left(\mathfrak{⿰}_{C Q-3}\right)=0.497265, \\
& \mathfrak{S}_{C Q}\left(\mathfrak{S}_{C Q-4}\right)=0.485264, \\
& \mathfrak{S}_{C Q}\left(\mathfrak{S}_{C Q-5}\right)=0.463763 .
\end{aligned}
$$

By LCQROFWG operator,

$$
\begin{aligned}
& \mathfrak{S}_{C Q}\left(\mathfrak{S}_{C Q-1}\right)=-0.11223, \\
& \mathfrak{S}_{C Q}\left(\mathfrak{S}_{C Q-2}\right)=-0.30462, \\
& \mathfrak{S}_{C Q}\left(\mathfrak{S}_{C Q-3}\right)=0.023319, \\
& \mathfrak{S}_{C Q}\left(\mathfrak{S}_{C Q-4}\right)=-0.29668, \\
& \mathfrak{S}_{C Q}\left(\mathfrak{S}_{C Q-5}\right)=-0.41047 .
\end{aligned}
$$

The graphical representation of these score values for each alternative is shown in Figure 2.

Step 4: we rank all alternatives and examine the best one such that

By LCQROFWA operator,

$$
\mathfrak{c}_{C Q-3} \geq \mathfrak{c}_{C Q-1} \geq \mathfrak{c}_{C Q-4} \geq \mathfrak{c}_{C Q-2} \geq \mathfrak{c}_{C Q-5} .
$$

By LCQROFWG operator,

$$
\mathfrak{c}_{C Q-3} \geq \mathfrak{c}_{C Q-1} \geq \mathfrak{c}_{C Q-4} \geq \mathfrak{c}_{C Q-2} \geq \mathfrak{c}_{C Q-5} .
$$

From this analysis, we compute that the best alternative is $\mathfrak{S}_{\mathrm{CQ}-3}$.

Example 3. Consider a decision-making issue, whose every entry is in the form of complex intuitionistic fuzzy numbers. In it, expect that an organization needs to make another item and they are investigating the ideal objective to acquire the most elevated advantages. For this, in the wake of dissecting the market, they have thought about the five potential techniques after their starter screening and are characterized as follows:

(1) $\mathfrak{S}_{C Q-1}$ : producing an invention adapted to the powerful businesses

(2) $\mathfrak{S}_{C Q-2}$ : producing an invention adapted to the midlevel businesses

(3) $\mathfrak{S}_{C Q-3}$ : producing an invention adapted to the lowlevel businesses

(4) $\mathfrak{S}_{C Q-4}$ : producing an invention adapted to all businesses

(5) $\mathfrak{S}_{\mathrm{CQ-5}}$ : not producing any invention

To assess these, the organization thinks about the practical factor as the key for the following year. Based on this considered alternative, they need to assess every methodology under the accompanying five general qualities:

(1) $\mathscr{C}_{C Q-1}$ : profits in the powerful conditions

(2) $\mathscr{C}_{C Q-2}$ : profits in the mid conditions

(3) $\mathscr{C}_{\mathrm{CQ-3}}$ : profits in the long conditions

(4) $\mathscr{C}_{C Q-4}$ : danger of the manufacture plan

(5) $\mathscr{C}_{\mathrm{CQ}-5}$ : other issues

Then, the steps of the investigated algorithm are discussed in the following ways :

Step 1: the rating values of each alternative are recorded in the form of complex intuitionistic fuzzy numbers 
TABLE 3: Rating values of the alternatives in terms of CPFNs for Example 2.

\begin{tabular}{|c|c|c|c|c|c|}
\hline Alternatives/attributes & $\mathscr{C}_{C Q-1}$ & $\mathscr{C}_{C Q-2}$ & $\mathscr{C}_{C Q-3}$ & $\mathscr{C}_{C Q-4}$ & $\mathscr{C}_{C Q-5}$ \\
\hline$C_{C Q-1}$ & $\left(\begin{array}{c}0.9 e^{i 2 \pi(0.8)} \\
0.1 e^{i 2 \pi(0.2)}\end{array}\right)$ & $\left(\begin{array}{c}0.91 e^{i 2 \pi(0.81)} \\
0.11 e^{i 2 \pi(0.21)}\end{array}\right)$ & $\left(\begin{array}{c}0.92 e^{i 2 \pi(0.82)} \\
0.12 e^{i 2 \pi(0.22)}\end{array}\right)$ & $\left(\begin{array}{c}0.93 e^{i 2 \pi(0.83)} \\
0.13 e^{i 2 \pi(0.23)}\end{array}\right)$ & $\left(\begin{array}{c}0.94 e^{i 2 \pi(0.84)} \\
0.14 e^{i 2 \pi(0.24)}\end{array}\right)$ \\
\hline$C_{C Q-2}$ & $\left(\begin{array}{c}0.8 e^{i 2 \pi(0.7)} \\
0.2 e^{i 2 \pi(0.3)}\end{array}\right)$ & $\left(\begin{array}{l}0.81 e^{i 2 \pi(0.71)} \\
0.21 e^{i 2 \pi(0.31)}\end{array}\right)$ & $\left(\begin{array}{c}0.82 e^{i 2 \pi(0.72)}, \\
0.22 e^{i 2 \pi(0.32)}\end{array}\right)$ & $\left(\begin{array}{c}0.83 e^{i 2 \pi(0.73)} \\
0.23 e^{i 2 \pi(0.33)}\end{array}\right)$ & $\left(\begin{array}{c}0.84 e^{i 2 \pi(0.74)} \\
0.24 e^{i 2 \pi(0.34)}\end{array}\right)$ \\
\hline$C_{C Q-3}$ & $\left(\begin{array}{c}0.9 e^{i 2 \pi(0.9)} \\
0.1 e^{i 2 \pi(0.1)}\end{array}\right)$ & $\left(\begin{array}{c}0.91 e^{i 2 \pi(0.91)} \\
0.11 e^{i 2 \pi(0.11)}\end{array}\right)$ & $\left(\begin{array}{c}0.92 e^{i 2 \pi(0.92)} \\
0.12 e^{i 2 \pi(0.12)}\end{array}\right)$ & $\left(\begin{array}{c}0.93 e^{i 2 \pi(0.93)} \\
0.13 e^{i 2 \pi(0.13)}\end{array}\right)$ & $\left(\begin{array}{c}0.94 e^{i 2 \pi(0.94)} \\
0.14 e^{i 2 \pi(0.14)}\end{array}\right)$ \\
\hline$C_{C Q-4}$ & $\left(\begin{array}{c}0.8 e^{i 2 \pi(0.8)} \\
0.2 e^{i 2 \pi(0.3)}\end{array}\right)$ & $\left(\begin{array}{l}0.81 e^{i 2 \pi(0.81)} \\
0.21 e^{i 2 \pi(0.31)}\end{array}\right)$ & $\left(\begin{array}{c}0.82 e^{i 2 \pi(0.82)}, \\
0.22 e^{i 2 \pi(0.32)}\end{array}\right)$ & $\left(\begin{array}{c}0.83 e^{i 2 \pi(0.83)} \\
0.23 e^{i 2 \pi(0.33)}\end{array}\right)$ & $\left(\begin{array}{c}0.84 e^{i 2 \pi(0.84)} \\
0.24 e^{i 2 \pi(0.34)}\end{array}\right)$ \\
\hline$C_{C Q-5}$ & $\left(\begin{array}{c}0.8 e^{i 2 \pi(0.5)} \\
0.3 e^{i 2 \pi(0.6)}\end{array}\right)$ & $\left(\begin{array}{c}0.81 e^{i 2 \pi(0.51)} \\
0.31 e^{i 2 \pi(0.61)}\end{array}\right)$ & $\left(\begin{array}{c}0.82 e^{i 2 \pi(0.52)} \\
0.32 e^{i 2 \pi(0.62)}\end{array}\right)$ & $\left(\begin{array}{c}0.83 e^{i 2 \pi(0.53)} \\
0.33 e^{i 2 \pi(0.63)}\end{array}\right)$ & $\left(\begin{array}{c}0.84 e^{i 2 \pi(0.54)} \\
0.34 e^{i 2 \pi(0.64)}\end{array}\right)$ \\
\hline
\end{tabular}

TABLE 4: Collective values by using LCQROFWA and LCQROFWG operators for Example 3.

\begin{tabular}{lcr}
\hline Alternatives & By LCQROFWA operator & By LCQROFWG operator \\
\hline$C_{C Q-1}$ & $\left(0.9952 e^{i 2 \pi(0.9960)}, 0.0028 e^{i 2 \pi(0.0101)}\right)$ & $\left(0.3030 e^{i 2 \pi(0.2360)}, 0.3284 e^{i 2 \pi(0.7425)}\right)$ \\
$C_{C Q-2}$ & $\left(0.9744 e^{i 2 \pi(0.9892)}, 0.0101 e^{i 2 \pi(0.0225)}\right)$ & $\left(0.2360 e^{i 2 \pi(0.1548)}, 0.7424 e^{i 2 \pi(0.8642)}\right)$ \\
$C_{C Q-3}$ & $\left(0.9952 e^{i 2 \pi(0.9992)}, 0.0028 e^{i 2 \pi(0.0028)}\right)$ & $\left(0.3930 e^{i 2 \pi(0.3930)}, 0.3284 e^{i 2 \pi(0.3284)}\right)$ \\
$C_{C Q-4}$ & $\left(0.9744 e^{i 2 \pi(0.9960)}, 0.0101 e^{i 2 \pi(0.0225)}\right)$ & $\left(0.2360 e^{i 2 \pi(0.2360)}, 0.7425 e^{i 2 \pi(0.8642)}\right)$ \\
$C_{C Q-5}$ & $\left(0.9744 e^{i 2 \pi(0.9573)}, 0.0225 e^{i 2 \pi(0.1027)}\right)$ & $\left(0.2360 e^{i 2 \pi(0.0665)}, 0.8642 e^{i 2 \pi(0.9773)}\right)$ \\
\hline
\end{tabular}

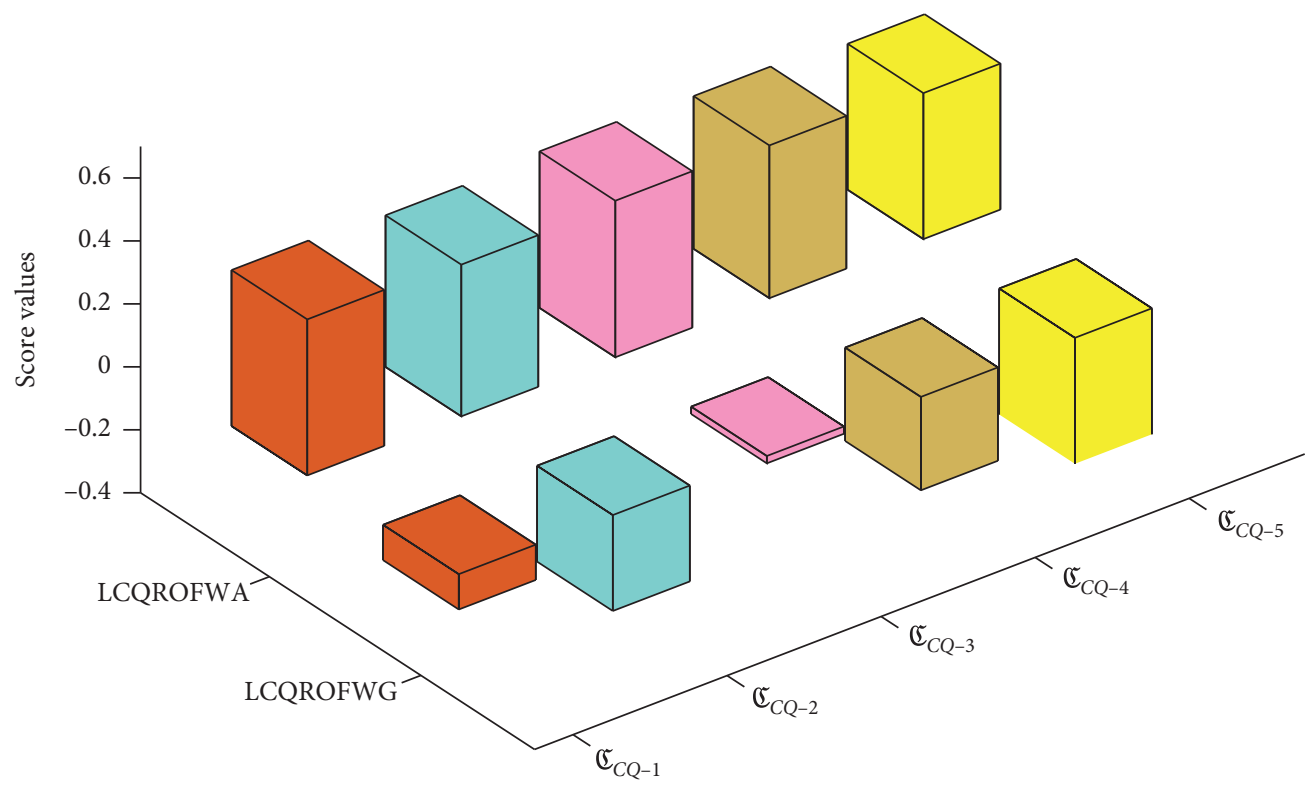

Figure 2: Geometrical representation of the score values of Example 2.

(CIFNs), which is a special case of the stated CQROFNs shown in Table 5.

Step 2: with weight vector $(0.3,0.3,0.2,0.1,0.1)$ and utilizing the LCQROFWA and LCQROFWG operators, we can obtain the aggregated values of each alternative. Their results are listed in Table 6.

Step 3: by using the score function, we find the score values of the aggregated values of Step 2 as follows:
By LCQROFWA operator,

$$
\begin{aligned}
& \mathfrak{\Im}_{C Q}\left(\mathfrak{S}_{C Q-1}\right)=0.048446 \text {, } \\
& \widetilde{\mathfrak{S}}_{\mathrm{CQ}}\left(\mathfrak{c}_{\mathrm{CQ}-2}\right)=0.0662 \text {, } \\
& \mathfrak{S}_{C Q}\left(\mathfrak{S}_{C Q-3}\right)=0.304077 \text {, } \\
& \mathfrak{\Im}_{C Q}\left(\mathfrak{S}_{C Q-4}\right)=0.178901 \text {, } \\
& \mathfrak{S}_{C Q}\left(\mathfrak{C}_{C Q-5}\right)=-0.01105 \text {. }
\end{aligned}
$$


TABle 5: Decision matrix in terms of CIFNs for Example 3.

\begin{tabular}{|c|c|c|c|c|c|}
\hline Alternatives/attributes & $\mathscr{C}_{C Q-1}$ & $\mathscr{C}_{C Q-2}$ & $\mathscr{C}_{\mathrm{CQ-3}}$ & $\mathscr{C}_{C Q-4}$ & $\mathscr{C}_{C Q-5}$ \\
\hline$C_{C Q-1}$ & $\left(\begin{array}{l}0.3 e^{i 2 \pi(0.4)} \\
0.1 e^{i 2 \pi(0.2)}\end{array}\right)$ & $\left(\begin{array}{c}0.31 e^{i 2 \pi(0.41)} \\
0.11 e^{i 2 \pi(0.21)}\end{array}\right)$ & $\left(\begin{array}{c}0.32 e^{i 2 \pi(0.42)} \\
0.12 e^{i 2 \pi(0.22)}\end{array}\right)$ & $\left(\begin{array}{c}0.33 e^{i 2 \pi(0.43)} \\
0.13 e^{i 2 \pi(0.23)}\end{array}\right)$ & $\left(\begin{array}{l}0.34 e^{i 2 \pi(0.44)} \\
0.14 e^{i 2 \pi(0.24)}\end{array}\right)$ \\
\hline$C_{C Q-2}$ & $\left(\begin{array}{l}0.4 e^{i 2 \pi(0.3)} \\
0.2 e^{i 2 \pi(0.3)}\end{array}\right)$ & $\left(\begin{array}{c}0.41 e^{i 2 \pi(0.31)} \\
0.21 e^{i 2 \pi(0.31)}\end{array}\right)$ & $\left(\begin{array}{c}0.42 e^{i 2 \pi(0.32)} \\
0.22 e^{i 2 \pi(0.32)}\end{array}\right)$ & $\left(\begin{array}{c}0.43 e^{i 2 \pi(0.33)} \\
0.23 e^{i 2 \pi(0.33)}\end{array}\right)$ & $\left(\begin{array}{l}0.44 e^{i 2 \pi(0.34)} \\
0.24 e^{i 2 \pi(0.34)}\end{array}\right)$ \\
\hline$C_{C Q-3}$ & $\left(\begin{array}{l}0.6 e^{i 2 \pi(0.7)} \\
0.1 e^{i 2 \pi(0.1)}\end{array}\right)$ & $\left(\begin{array}{c}0.61 e^{i 2 \pi(0.71)} \\
0.11 e^{i 2 \pi(0.11)}\end{array}\right)$ & $\left(\begin{array}{l}0.62 e^{i 2 \pi(0.72)} \\
0.12 e^{i 2 \pi(0.12)}\end{array}\right)$ & $\left(\begin{array}{l}0.63 e^{i 2 \pi(0.73)} \\
0.13 e^{i 2 \pi(0.13)}\end{array}\right)$ & $\left(\begin{array}{l}0.64 e^{i 2 \pi(0.74)} \\
0.14 e^{i 2 \pi(0.14)}\end{array}\right)$ \\
\hline$C_{\mathrm{CQ}-4}$ & $\left(\begin{array}{c}0.5 e^{i 2 \pi(0.5)} \\
0.2 e^{i 2 \pi(0.3)}\end{array}\right)$ & $\left(\begin{array}{c}0.51 e^{i 2 \pi(0.51)} \\
0.21 e^{i 2 \pi(0.31)}\end{array}\right)$ & $\left(\begin{array}{c}0.52 e^{i 2 \pi(0.52)} \\
0.22 e^{i 2 \pi(0.32)}\end{array}\right)$ & $\left(\begin{array}{c}0.53 e^{i 2 \pi(0.53)} \\
0.23 e^{i 2 \pi(0.33)}\end{array}\right)$ & $\left(\begin{array}{l}0.54 e^{i 2 \pi(0.54)} \\
0.24 e^{i 2 \pi(0.34)}\end{array}\right)$ \\
\hline$C_{C Q-5}$ & $\left(\begin{array}{c}0.4 e^{i 2 \pi(0.3)} \\
0.3 e^{i 2 \pi(0.6)}\end{array}\right)$ & $\left(\begin{array}{c}0.41 e^{i 2 \pi(0.31)} \\
0.31 e^{i 2 \pi(0.61)}\end{array}\right)$ & $\left(\begin{array}{c}0.42 e^{i 2 \pi(0.32)} \\
0.32 e^{i 2 \pi(0.62)}\end{array}\right)$ & $\left(\begin{array}{c}0.43 e^{i 2 \pi(0.33)} \\
0.33 e^{i 2 \pi(0.63)}\end{array}\right)$ & $\left(\begin{array}{l}0.44 e^{i 2 \pi(0.34)} \\
0.34 e^{i 2 \pi(0.64)^{\prime}}\end{array}\right)$ \\
\hline
\end{tabular}

TABLE 6: Aggregated values by using LCQROFWA and LCQROFWG operators for Example 3.

\begin{tabular}{lcr}
\hline Alternatives & By LCQROFWA operator & By LCQROFWG operator \\
\hline$C_{C Q-1}$ & $\left(0.2666 e^{i 2 \pi(0.6170)}, 0.0522 e^{i 2 \pi(0.1044)}\right)$ & $\left(0.1635 e^{i 2 \pi(0.2320)}, 0.0554 e^{i 2 \pi(0.3301)}\right)$ \\
$C_{C Q-2}$ & $\left(0.0358 e^{i 2 \pi(0.4968)}, 0.1044 e^{i 2 \pi(0.1635)}\right)$ & $\left(0.2320 e^{i 2 \pi(0.1635)}, 0.3301 e^{i 2 \pi(0.4968)}\right)$ \\
$C_{C Q-3}$ & $\left(0.4669 e^{i 2 \pi(0.8538)}, 0.0522 e^{i 2 \pi(0.0522)}\right)$ & $\left(0.4134 e^{i 2 \pi(0.5437)}, 0.0554 e^{i 2 \pi(0.0554)}\right)$ \\
$C_{C Q-4}$ & $\left(0.2725 e^{i 2 \pi(0.7110)}, 0.1044 e^{i 2 \pi(0.1635)}\right)$ & $\left(0.3133 e^{i 2 \pi(0.3133)}, 0.3301 e^{i 2 \pi(0.4968)}\right)$ \\
$C_{C Q-5}$ & $\left(0.0358 e^{i 2 \pi(0.4968)}, 0.1635 e^{i 2 \pi(0.1434)}\right)$ & $\left(0.2320 e^{i 2 \pi(0.1635)}, 0.4968 e^{i 2 \pi(0.7882)}\right)$ \\
\hline
\end{tabular}

By LCQROFWG operator,

$$
\begin{aligned}
& \mathfrak{S}_{C Q}\left(\mathfrak{c}_{C Q-1}\right)=0.002485, \\
& \mathfrak{S}_{C Q}\left(\mathfrak{c}_{C Q-2}\right)=-0.10787, \\
& \mathfrak{S}_{C Q}\left(\mathfrak{c}_{C Q-3}\right)=0.211543, \\
& \mathfrak{S}_{C Q}\left(\mathfrak{夭}_{C Q-4}\right)=-0.0501, \\
& \mathfrak{\Im}_{C Q}\left(\mathfrak{c}_{C Q-5}\right)=-0.2224 .
\end{aligned}
$$

However, the graphical representation of these score values for each alternative with respect to LCQROFWA and LCQROFWG operators is shown in Figure 3.

Step 4: we rank all alternatives and examine the best one such that

By LCQROFWA operator,

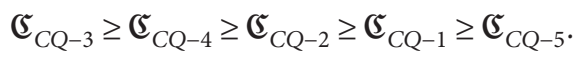

By LCQROFWG operator,

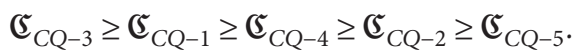

And hence the best alternative obtained is $\mathfrak{E}_{C Q-3}$

5.3. Comparative Analysis. In this section, we establish a comparison of the proposed operators based on complex q-rung orthopair fuzzy environment and the existing operations under different environments such as IFS, PFS, QROFS, and CQROFS. To address this, we have taken the dataset as described in Examples 1-3 and implemented the existing approaches on it. The considered studies for the comparative analysis are as follows: $\mathrm{Xu}$ [33] investigated the aggregation operators based on IFSs, Garg [12] developed aggregation operators based on PFSs, Liu and Wang [18] explored aggregation operators for QROFSs, Garg and Rani [26] investigated aggregation operators for CIFSs, and Liu et al. [31] proposed aggregation operators for CQROFSs. A detailed analysis of the results obtained by the proposed study and existing study is given in Tables 7-9 by using the information of Tables 1,3 , and 5 , respectively. The geometrical representations of the investigated score values of Tables 7-9 are discussed in Figures 4-6, respectively.

From Tables $7-9$, it is clearly indicated that the existing operators under the environment of IFSs, CIFSs, PFSs, CPFSs, and QROFSs failed to aggregate the information provided in complex q-rung orthopair fuzzy environment which shows the superiority of stated operators of CQROFSs. As we also discussed in Section 4, existing operators are the special cases of operators of CQROFSs. Also, from the computed results and the stated theory, we conclude the following advantages and observations about the present work:

(1) For $q_{C Q}=2$, the investigated operators based on CQROFSs are converted for CPFSs

(2) For $q_{C Q}=1$, the investigated operators based on CQROFSs are converted for CIFSs

(3) For $q_{C Q}=2$ with the imaginary part being zero, the investigated operators based on CQROFSs are converted for PFSs 


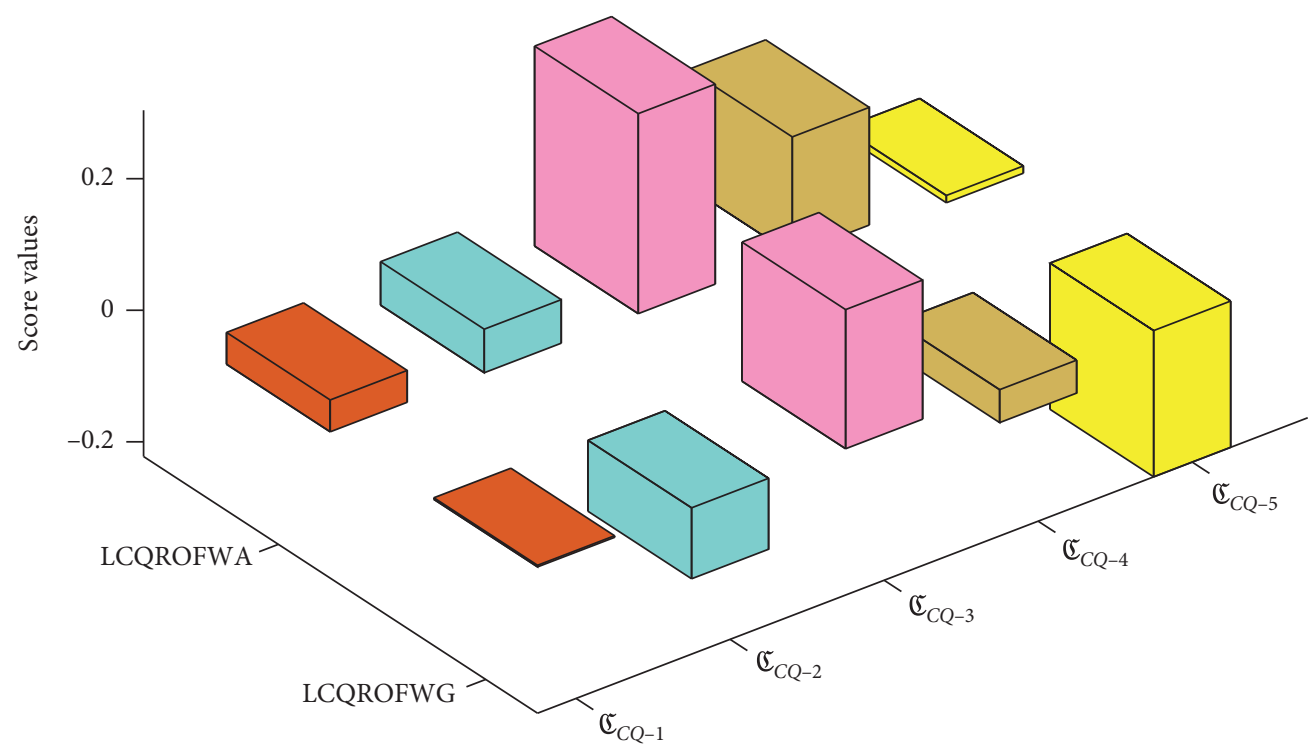

FIGURE 3: Geometrical representation of the alternatives discussed in Example 3.

TABLE 7: Comparative analysis for the information corresponding to Example 1.

\begin{tabular}{|c|c|c|c|}
\hline Methods & Operators & Score values of the alternatives & Ranking order \\
\hline \multirow{2}{*}{$\mathrm{Xu}[33]$} & WA & Cannot be calculated & Cannot be calculated \\
\hline & WG & Cannot be calculated & Cannot be calculated \\
\hline \multirow{2}{*}{ Garg [12] } & WA & Cannot be calculated & Cannot be calculated \\
\hline & WG & Cannot be calculated & Cannot be calculated \\
\hline \multirow{2}{*}{ Liu and Wang [18] } & WA & Cannot be calculated & Cannot be calculated \\
\hline & WG & Cannot be calculated & Cannot be calculated \\
\hline \multirow{2}{*}{ Garg and Rani [26] } & WA & Cannot be calculated & Cannot be calculated \\
\hline & WG & Cannot be calculated & Cannot be calculated \\
\hline \multirow{2}{*}{ Liu et al. [31] } & WA & Cannot be calculated & Cannot be calculated \\
\hline & WG & Cannot be calculated & Cannot be calculated \\
\hline \multirow{2}{*}{ Proposed operators } & WA & 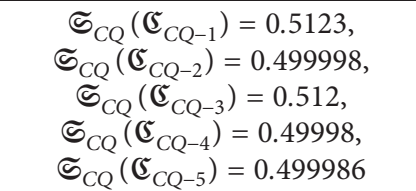 & 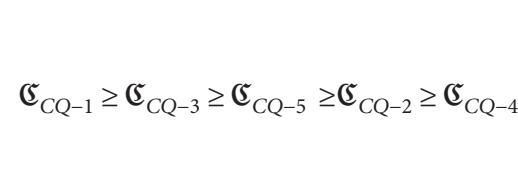 \\
\hline & WG & $\begin{array}{c}\mathfrak{S}_{C Q}\left(\mathfrak{S}_{C Q-1}\right)=-0.5123, \\
\mathfrak{S}_{C Q}\left(\mathfrak{S}_{C Q-2}\right)=-0.5121, \\
\mathfrak{S}_{C Q}\left(\mathfrak{S}_{C Q-3}\right)=-0.4991, \\
\mathfrak{S}_{C Q}\left(\mathfrak{S}_{C Q-4}\right)=-0.512, \\
\mathfrak{S}_{C Q}\left(\mathfrak{S}_{C Q-5}\right)=-0.49988\end{array}$ & 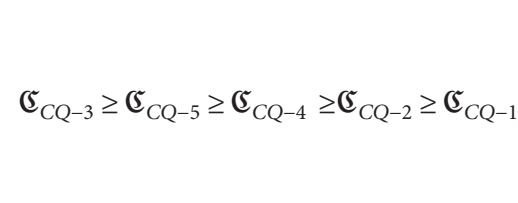 \\
\hline
\end{tabular}

WA: weighted average; WG: weighted geometric.

TABLE 8: Comparative analysis for the information of Table 3 corresponding to Example 2.

\begin{tabular}{lccc}
\hline Methods & Operators & Score values & Ranking values \\
\hline \multirow{2}{*}{$\mathrm{Xu}[33]$} & WA & Cannot be calculated & Cannot be calculated \\
& WG & Cannot be calculated & Cannot be calculated \\
\hline \multirow{2}{*}{ Garg [12] } & WA & Cannot be calculated & Cannot be calculated \\
\hline \multirow{2}{*}{ Liu and Wang [18] } & WG & Cannot be calculated & Cannot be calculated \\
& WA & Cannot be calculated & Cannot be calculated \\
\hline \multirow{2}{*}{ Garg and Rani [26] } & WG & Cannot be calculated & Cannot be calculated \\
& WG & Cannot be calculated & Cannot be calculated \\
\hline
\end{tabular}


TABle 8: Continued.

\begin{tabular}{|c|c|c|c|}
\hline Methods & Operators & Score values & Ranking values \\
\hline \multirow{2}{*}{ Liu et al. [31] } & WA & $\begin{array}{l}\mathfrak{S}_{C Q}\left(\mathfrak{S}_{C Q-1}\right)=0.721174, \\
\mathfrak{S}_{C Q}\left(\mathfrak{S}_{C Q-2}\right)=0.514836, \\
\mathfrak{S}_{C Q}\left(\mathfrak{S}_{C Q-3}\right)=0.824551, \\
\mathfrak{S}_{C Q}\left(\mathfrak{G}_{C Q-4}\right)=0.591397, \\
\mathfrak{S}_{C Q}\left(\mathfrak{c}_{C Q-5}\right)=0.226322\end{array}$ & 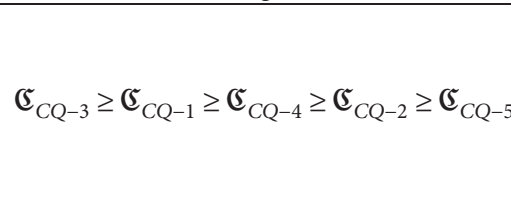 \\
\hline & WG & 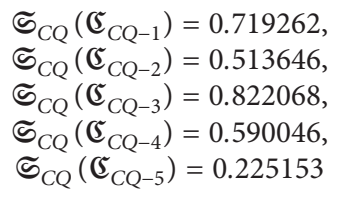 & $\mathfrak{夭}_{C Q-3} \geq \mathfrak{夭}_{C Q-1} \geq \mathfrak{c}_{C Q-4} \geq \mathfrak{c}_{C Q-2} \geq \mathfrak{c}_{C Q-5}$ \\
\hline \multirow{2}{*}{$\begin{array}{l}\text { Proposed } \\
\text { operators }\end{array}$} & WA & $\begin{array}{l}\mathfrak{S}_{C Q}\left(\mathfrak{S}_{C Q-1}\right)=0.495626, \\
\mathfrak{S}_{C Q}\left(\mathfrak{S}_{C Q-2}\right)=0.481911, \\
\mathfrak{S}_{C Q}\left(\mathfrak{S}_{C Q-3}\right)=0.497265, \\
\mathfrak{S}_{C Q}\left(\mathfrak{G}_{C Q-4}\right)=0.485264, \\
\mathfrak{S}_{C Q}\left(\mathfrak{c}_{C Q-5}\right)=0.463763\end{array}$ & 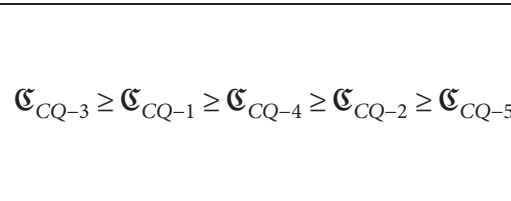 \\
\hline & WG & 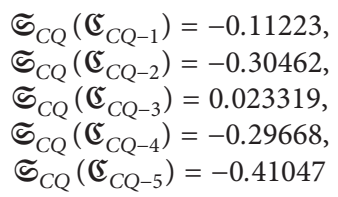 & 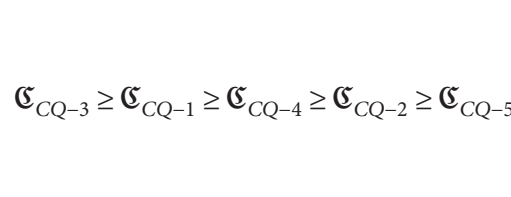 \\
\hline
\end{tabular}

WA: weighted average; WG: weighted geometric.

TAвLE 9: Comparative analysis for the information of Table 5 corresponding to Example 3.

\begin{tabular}{|c|c|c|c|}
\hline Methods & Operators & Score values & Ranking values \\
\hline $\mathrm{Xu}[33]$ & $\begin{array}{l}\text { WA } \\
\text { WG }\end{array}$ & $\begin{array}{l}\text { Cannot be calculated } \\
\text { Cannot be calculated }\end{array}$ & $\begin{array}{l}\text { Cannot be calculated } \\
\text { Cannot be calculated }\end{array}$ \\
\hline Garg [12] & $\begin{array}{l}\text { WA } \\
\text { WG }\end{array}$ & $\begin{array}{l}\text { Cannot be calculated } \\
\text { Cannot be calculated }\end{array}$ & $\begin{array}{l}\text { Cannot be calculated } \\
\text { Cannot be calculated }\end{array}$ \\
\hline Liu and Wang [18] & $\begin{array}{l}\text { WA } \\
\text { WG }\end{array}$ & $\begin{array}{l}\text { Cannot be calculated } \\
\text { Cannot be calculated }\end{array}$ & $\begin{array}{l}\text { Cannot be calculated } \\
\text { Cannot be calculated }\end{array}$ \\
\hline \multirow{2}{*}{ Garg and Rani [26] } & WA & 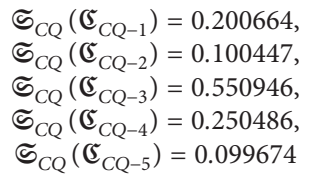 & $\mathfrak{夭}_{C Q-3} \geq \mathfrak{G}_{C Q-4} \geq \mathfrak{G}_{C Q-1} \geq \mathfrak{夭}_{C Q-2} \geq \mathfrak{C}_{C Q-5}$ \\
\hline & WG & $\begin{array}{l}\mathfrak{S}_{C Q}\left(\mathfrak{c}_{C Q-1}\right)=0.199675 \\
\mathfrak{S}_{C Q}\left(\mathfrak{c}_{C Q-2}\right)=0.099661, \\
\mathfrak{S}_{C Q}\left(\mathfrak{c}_{C Q-3}\right)=0.549784 \\
\mathfrak{S}_{C Q}\left(\mathfrak{c}_{C Q-4}\right)=0.249729, \\
\mathfrak{S}_{C Q}\left(\mathfrak{c}_{C Q-5}\right)=0.100394\end{array}$ & $\mathfrak{夭}_{C Q-3} \geq \mathfrak{夭}_{C Q-4} \geq \mathfrak{夭}_{C Q-1} \geq \mathfrak{c}_{C Q-5} \geq \mathfrak{夭}_{C Q-2}$ \\
\hline \multirow{2}{*}{ Liu et al. [31] } & WA & $\begin{array}{l}\mathfrak{S}_{C Q}\left(\mathfrak{c}_{C Q-1}\right)=0.105976 \\
\mathfrak{S}_{C Q}\left(\mathfrak{c}_{C Q-2}\right)=0.063178 \\
\mathfrak{S}_{C Q}\left(\mathfrak{c}_{C Q-3}\right)=0.430999 \\
\mathfrak{S}_{C Q}\left(\mathfrak{c}_{C Q-4}\right)=0.192445, \\
\mathfrak{S}_{C Q}\left(\mathfrak{c}_{C Q-5}\right)=0.102421\end{array}$ & $\mathfrak{夭}_{C Q-3} \geq \mathfrak{G}_{C Q-4} \geq \mathfrak{G}_{C Q-1} \geq \mathfrak{C}_{C Q-5} \geq \mathfrak{C}_{C Q-2}$ \\
\hline & WG & $\begin{array}{l}\mathfrak{S}_{C Q}\left(\mathfrak{c}_{C Q-1}\right)=0.105264, \\
\mathfrak{S}_{C Q}\left(\mathfrak{c}_{C Q-2}\right)=0.062448 \\
\mathfrak{S}_{C Q}\left(\mathfrak{c}_{C Q-3}\right)=0.430069, \\
\mathfrak{S}_{C Q}\left(\mathfrak{c}_{C Q-4}\right)=0.191647, \\
\mathfrak{S}_{C Q}\left(\mathfrak{S}_{C Q-5}\right)=0.103246\end{array}$ & 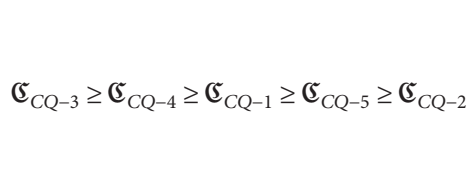 \\
\hline \multirow{2}{*}{$\begin{array}{l}\text { Proposed } \\
\text { operators }\end{array}$} & WA & $\begin{array}{l}\mathfrak{S}_{C Q}\left(\mathfrak{S}_{C Q-1}\right)=0.048446, \\
\mathfrak{S}_{C Q}\left(\mathfrak{S}_{C Q-2}\right)=0.06620, \\
\mathfrak{S}_{C Q}\left(\mathfrak{S}_{C Q-3}\right)=0.304077 \\
\mathfrak{S}_{C Q}\left(\mathfrak{S}_{C Q-4}\right)=0.178901, \\
\mathfrak{S}_{C Q}\left(\mathfrak{S}_{C Q-5}\right)=-0.01105\end{array}$ & $\mathfrak{c}_{C Q-3} \geq \mathfrak{夭}_{C Q-4} \geq \mathfrak{c}_{C Q-2} \geq \mathfrak{c}_{C Q-1} \geq \mathfrak{V}_{C Q-5}$ \\
\hline & WG & $\begin{array}{l}\mathfrak{S}_{C Q}\left(\mathfrak{S}_{C Q-1}\right)=0.002485, \\
\mathfrak{S}_{C Q}\left(\mathfrak{c}_{C Q-2}\right)=-0.10787, \\
\mathfrak{S}_{C Q}\left(\mathfrak{c}_{C Q-3}\right)=0.211543, \\
\mathfrak{S}_{C Q}\left(\mathfrak{c}_{C Q-4}\right)=-0.0501, \\
\mathfrak{S}_{C Q}\left(\mathfrak{c}_{C Q-5}\right)=-0.2224\end{array}$ & $\mathfrak{夭}_{C Q-3} \geq \mathfrak{夭}_{C Q-1} \geq \mathfrak{夭}_{C Q-4} \geq \mathfrak{夭}_{C Q-2} \geq \mathfrak{夭}_{C Q-5}$ \\
\hline
\end{tabular}

WA: weighted average; WG: weighted geometric. 


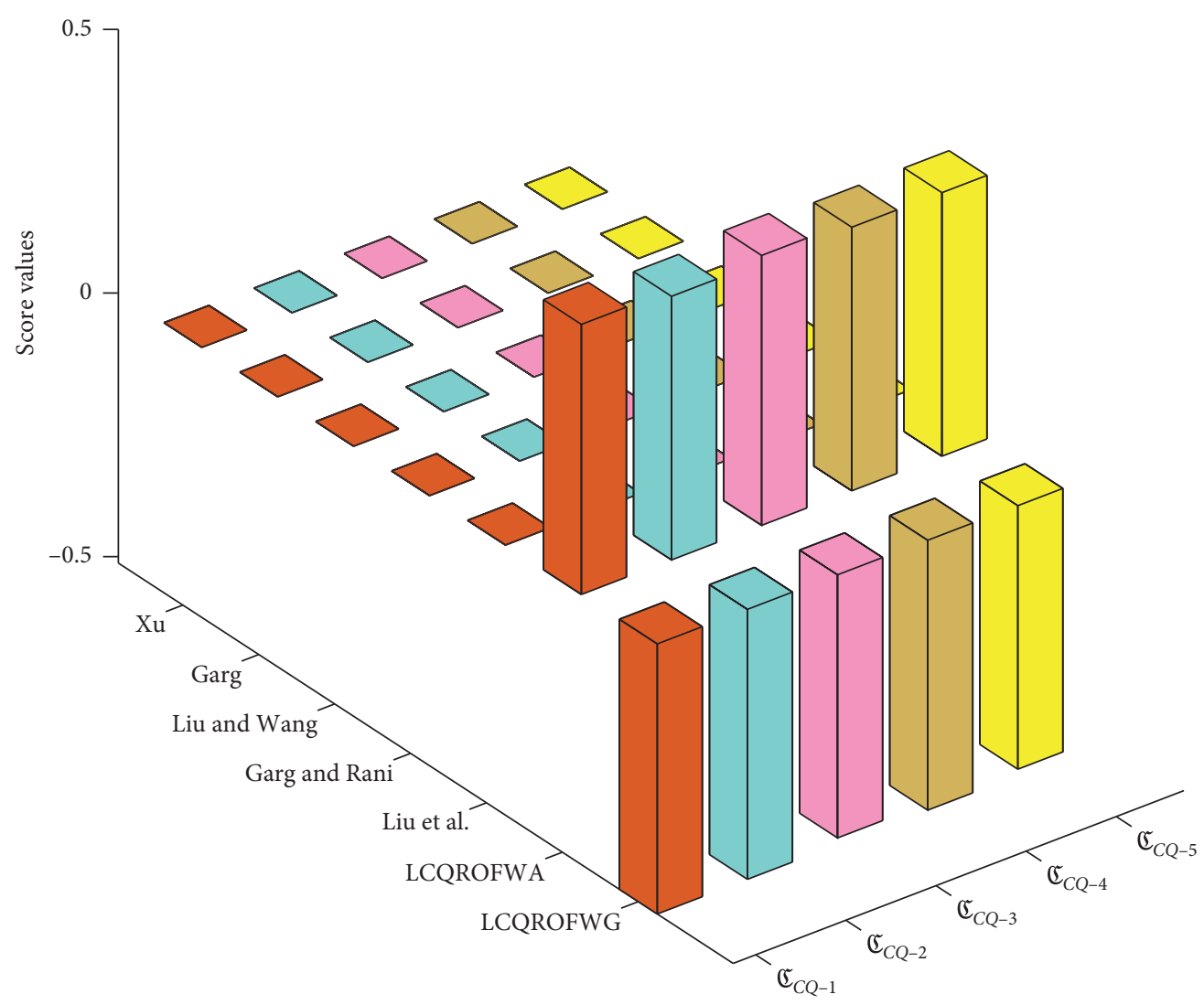

FIGURE 4: Graphical representations of the score values of the alternatives given in Table 7.

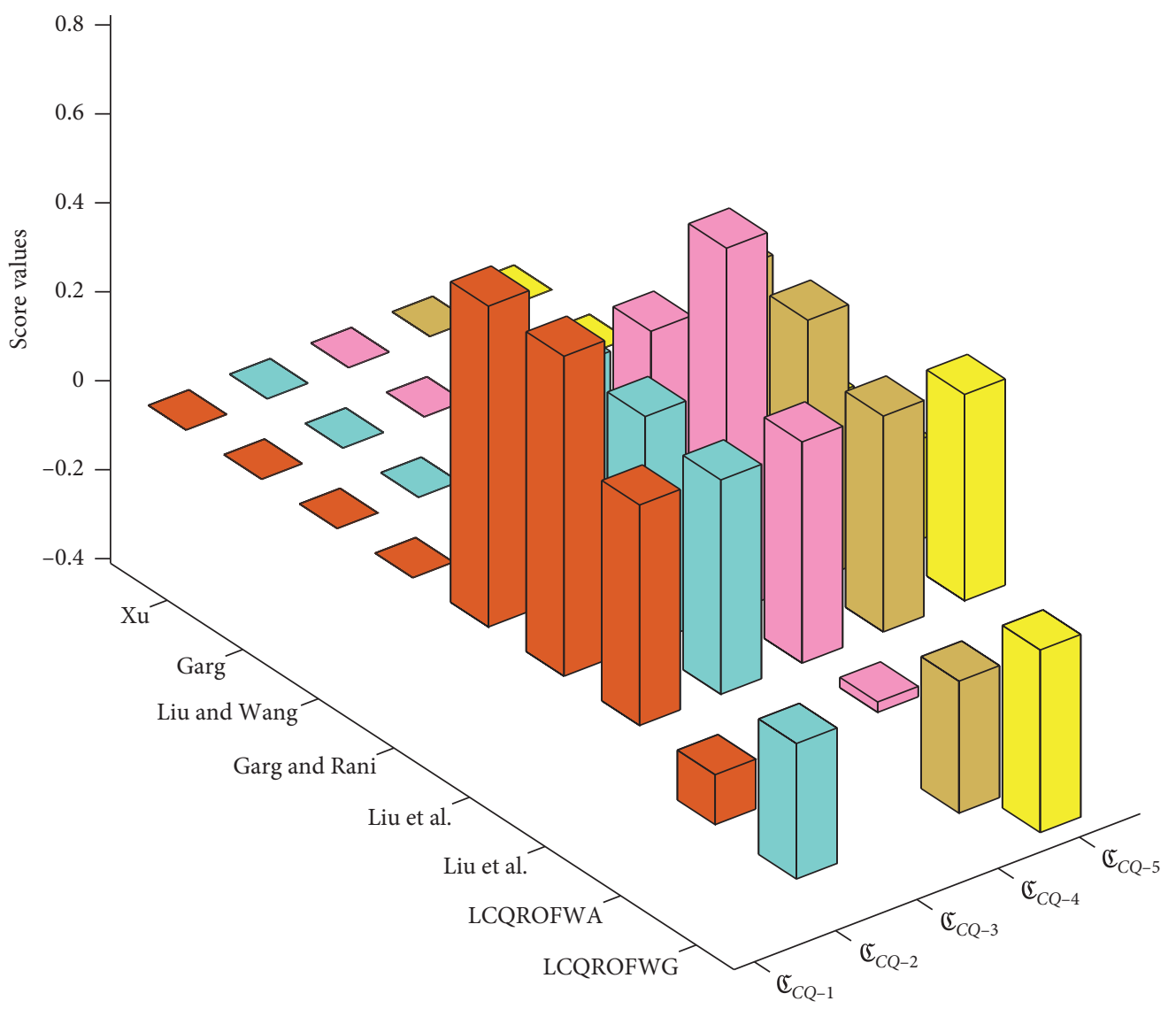

FigURE 5: Graphical representations of the score values of the alternatives given in Table 8 . 


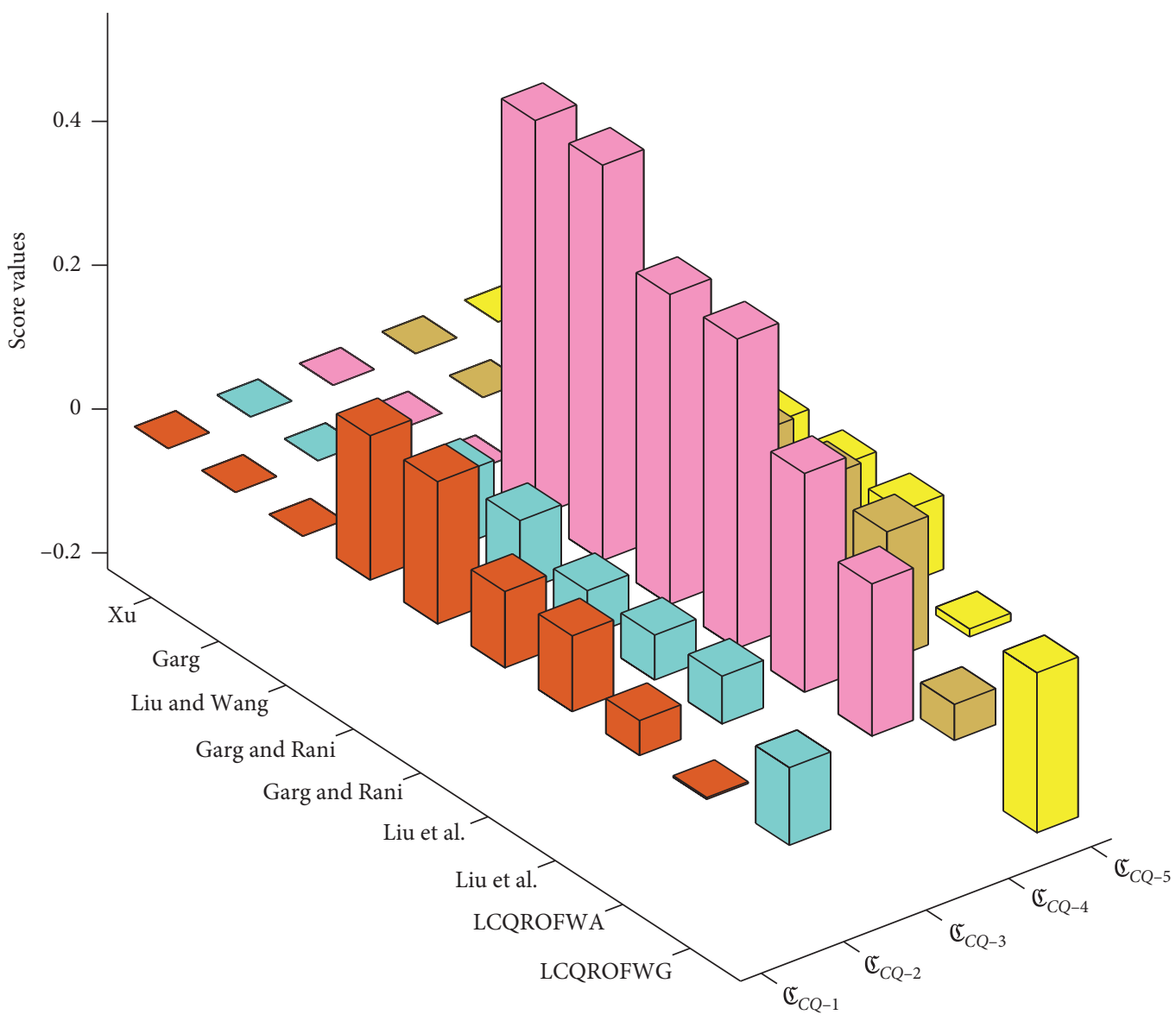

FIgURE 6: Geometrical representation of the score values of the alternatives given in Table 9.

(4) For $q_{C Q}=1$ with imaginary part being zero, the investigated operators based on CQROFSs is converted for IFSs

From the above analysis, we obtain the result that the investigated operators based on CQROFSs are extensively useful and validated compared to other existing ideas [21-27].

\section{Conclusion}

A complex q-rung orthopair fuzzy set (CQROFS) is one of the useful tools to handle the uncertainties in the data compared to the other existing sets such as IFSs, PFSs, and QROFSs. The main characteristic of the CQROFS is that it handles the imprecise information using four membership degrees such that the sum of the q-powers of the real parts (also for imaginary parts) of the membership and nonmembership degrees is restricted to the unit interval. In the decisionmaking process, the aggregation operators offer a flexible arrangement with its modifiable factors because of the algebraic general structure. On the other hand, aggregation operators perform a significant role in conveying the magnitude level of options and characteristics. In this study, we develop some new LOLs between the pairs of the CQROFS. Based on these LOLs, we developed different aggregator operators, namely, LCQROFQA and LCQROFQG, and investigated their properties. Also, a MADM technique is investigated by considering the developed operators. Some examples are illustrated with the help of discovered operators to find the validity and consistency of the investigated approaches. Finally, to improve the quality of the explored approaches, comparative analysis, advantages, and graphical representations are also demonstrated. In the future, we will extend the investigated approaches in different fuzzy environments to solve the problems related to decision-making, consensus problems, and so forth [34-41].

\section{Data Availability}

No data were used to support this study.

\section{Conflicts of Interest}

The authors declare that they have no conflicts of interest.

\section{Acknowledgments}

The authors are grateful for the financial help provided by Taif University Researchers Supporting Project (no. TURSP2020/73), Taif University, Taif, Saudi Arabia. 


\section{References}

[1] H. Garg, "CN-q-ROFS: connection number-based q-rung orthopair fuzzy set and their application to decision-making process," International Journal of Intelligent Systems, vol. 36, no. 7, pp. 3106-3143, 2021.

[2] K. Atanassov, "Intuitionistic fuzzy sets," International Journal Bioautomation, vol. 20, p. 1, 2016.

[3] L. A. Zadeh, "Fuzzy sets," Information and control, vol. 8, no. 3, pp. 338-353, 1965.

[4] K. T. Atanassov, "Interval valued intuitionistic fuzzy sets," in Intuitionistic Fuzzy SetsPhysica, Heidelberg, Germany, 1999.

[5] K. Rahman, A. Sanan, A. Saleem, and Y. K. Muhammad, "Some induced generalized Einstein aggregating operators and their application to group decision-making problem using intuitionistic fuzzy numbers," Annals of Optimization Theory \& Practices, vol. 3, no. 3, pp. 15-49, 2020.

[6] D. Yu, "Group decision making based on generalized intuitionistic fuzzy prioritized geometric operator," International Journal of Intelligent Systems, vol. 27, no. 7, pp. 635-661, 2012.

[7] P. A. Ejegwa, I. C. Onyeke, and V. Adah, "An algorithm for an improved intuitionistic fuzzy correlation measure with medical diagnostic application," Annals of Optimization Theory \& Practices, vol. 3, no. 3, pp. 51-68, 2020.

[8] K. Bhattacharya and S. K. De, "Decision making under intuitionistic fuzzy metric distances," Annals of Optimization Theory \& Practices, vol. 3, no. 2, pp. 49-64, 2020.

[9] R. R. Yager, "Pythagorean fuzzy subsets," in Proceedings of the 2013 Joint IFSA World Congress and NAFIPS Annual Meeting (IFSA/NAFIPS), pp. 57-61, IEEE, Edmonton, Canada, June 2013.

[10] X. Peng and H. Yuan, "Pythagorean fuzzy multi-criteria decision making method based on multiparametric similarity measure," Cognitive Computation, vol. 13, no. 2, pp. 466-484, 2021.

[11] H. Garg, "Sine trigonometric operational laws and its based Pythagorean fuzzy aggregation operators for group decisionmaking process," Artificial Intelligence Review, vol. 54, no. 6, pp. 4421-4447, 2021.

[12] P. Talukdar, S. Goala, P. Dutta, and B. Limboo, "Fuzzy multicriteria decision making in medical diagnosis using an advanced distance measure on linguistic Pythagorean fuzzy sets," Annals of Optimization Theory \& Practices, vol. 3, no. 4, pp. 113-131, 2020.

[13] L. Wang, H. Garg, and N. Li, "Pythagorean fuzzy interactive Hamacher power aggregation operators for assessment of express service quality with entropy weight," Soft Computing, vol. 25, no. 2, pp. 973-993, 2021.

[14] H. Garg, "New logarithmic operational laws and their aggregation operators for Pythagorean fuzzy set and their applications," International Journal of Intelligent Systems, vol. 34, no. 1, pp. 82-106, 2019.

[15] X. Peng and X. Ma, "Pythagorean fuzzy multi-criteria decision making method based on CODAS with new score function," Journal of Intelligent and Fuzzy Systems, vol. 38, no. 3, pp. 3307-3318, 2020.

[16] R. R. Yager, "Generalized orthopair fuzzy sets," IEEE Transactions on Fuzzy Systems, vol. 25, no. 5, pp. 1222-1230, 2016.

[17] H. Garg, "A novel trigonometric operation-based q-rung orthopair fuzzy aggregation operator and its fundamental properties," Neural Computing \& Applications, vol. 32, no. 18, pp. 15077-15099, 2020.
[18] P. Liu and P. Wang, "Some q-rung orthopair fuzzy aggregation operators and their applications to multiple-attribute decision making," International Journal of Intelligent Systems, vol. 33, no. 2, pp. 259-280, 2018.

[19] P. Liu, S. M. Chen, and P. Wang, "Multiple-attribute group decision-making based on q-rung orthopair fuzzy power Maclaurin symmetric mean operators," IEEE Transactions on Systems, Man, and Cybernetics: Systems, vol. 50, no. 10, pp. 3741-3756, 2020.

[20] M. J. Khan, P. Kumam, and M. Shutaywi, "Knowledge measure for the q-rung orthopair fuzzy sets," International Journal of Intelligent Systems, vol. 36, no. 2, pp. 628-655, 2021.

[21] A. M. D. J. S. Alkouri and A. R. Salleh, "Complex intuitionistic fuzzy sets," AIP Conference Proceedings, vol. 1482, no. 1, pp. 464-470, 2012

[22] D. Ramot, R. Milo, M. Friedman, and A. Kandel, "Complex fuzzy sets," IEEE Transactions on Fuzzy Systems, vol. 10, no. 2, pp. 171-186, 2002.

[23] Y. Rong, Y. Liu, and Z. Pei, "Complex q-rung orthopair fuzzy 2-tuple linguistic Maclaurin symmetric mean operators and its application to emergency program selection," International Journal of Intelligent Systems, vol. 35, no. 11, pp. 1749-1790, 2020.

[24] M. M. Khalaf, S. O. Alharbi, and W. Chammam, "Similarity measures between temporal complex intuitionistic fuzzy sets and application in pattern recognition and medical diagnosis," Discrete Dynamics in Nature and Society, vol. 2019, pp. 1-16, 2019.

[25] P. Liu, M. Akram, and A. Sattar, "Extensions of prioritized weighted aggregation operators for decision-making under complex q-rung orthopair fuzzy information," Journal of Intelligent and Fuzzy Systems, vol. 39, no. 5, pp. 7469-7493, 2020.

[26] H. Garg and D. Rani, "Novel aggregation operators and ranking method for complex intuitionistic fuzzy sets and their applications to decision-making process," Artificial Intelligence Review, vol. 53, no. 5, pp. 3595-3620, 2020.

[27] D. Rani and H. Garg, "Complex intuitionistic fuzzy preference relations and their applications in individual and group decision-making problems," International Journal of Intelligent Systems, vol. 36, no. 4, pp. 1800-1830, 2021.

[28] K. Ullah, T. Mahmood, Z. Ali, and N. Jan, "On some distance measures of complex Pythagorean fuzzy sets and their applications in pattern recognition," Complex \& Intelligent Systems, vol. 6, no. 1, pp. 15-27, 2020.

[29] M. Akram and S. Naz, "A novel decision-making approach under complex Pythagorean fuzzy environment," Mathematical and Computational Applications, vol. 24, no. 3, p. 73, 2019.

[30] M. Akram and A. Sattar, "Competition Graphs under complex Pythagorean fuzzy information," Journal of Applied Mathematics and Computing, vol. 63, no. 1-2, pp. 543-583, 2020.

[31] P. Liu, T. Mahmood, and Z. Ali, "Complex q-rung orthopair fuzzy aggregation operators and their applications in multiattribute group decision making," Information, vol. 11, no. 1, p. $5,2020$.

[32] H. Garg, Z. Ali, Z. Yang, T. Mahmood, and S. Aljahdali, "Multi-criteria decision-making algorithm based on aggregation operators under the complex interval-valued q-rung orthopair uncertain linguistic information," Journal of Intelligent and Fuzzy Systems, vol. 41, no. 1, pp. 1627-1656, 2021. 
[33] Z. Zeshui Xu, "Intuitionistic fuzzy aggregation operators," IEEE Transactions on Fuzzy Systems, vol. 15, no. 6, pp. 1179-1187, 2007.

[34] H. Garg and S.-M. Chen, "Multiattribute group decision making Based on Neutrality aggregation operators of q-rung orthopair fuzzy sets," Information Sciences, vol. 517, pp. 427-447, 2020.

[35] Z. Ali, T. Mahmood, and M.-S. Yang, "TOPSIS method based on complex spherical fuzzy sets with Bonferroni mean operators," Mathematics, vol. 8, no. 10, p. 1739, 2020.

[36] B. Batool, M. Ahmad, S. Abdullah, S. Ashraf, and R. Chinram, "Entropy based pythagorean probabilistic hesitant fuzzy decision making technique and its application for fog-haze factor Assessment problem," Entropy, vol. 22, no. 3, p. 318, 2020.

[37] B. Batool, S. S. Abosuliman, S. Abdullah, and S. Ashraf, "EDAS method for decision support modeling under the Pythagorean probabilistic hesitant fuzzy aggregation information," Journal of Ambient Intelligence and Humanized Computing, pp. 1-14, 2021.

[38] Q. Wu, W. Lin, L. Zhou, Y. Chen, and H. Chen, "Enhancing multiple attribute group decision making flexibility based on information fusion technique and hesitant Pythagorean fuzzy sets," Computers \& Industrial Engineering, vol. 127, pp. 954-970, 2019.

[39] Z. Zhang, J. Gao, Y. Gao, and W. Yu, "Two-sided matching decision making with multi-granular hesitant fuzzy linguistic term sets and incomplete criteria weight information," Expert Systems with Applications, vol. 168, Article ID 114311, 2021.

[40] Z. Zhang, Z. Li, and Y. Gao, "Consensus reaching for group decision making with multi-granular unbalanced linguistic information: a bounded confidence and minimum adjustment-based approach," Information Fusion, vol. 74, pp. 96110, 2021.

[41] A. Fahmi, F. Amin, and S. B. H. Shah, "Geometric operators based on linguistic interval-valued intuitionistic neutrosophic fuzzy number and their application in decision making," Annals of Optimization Theory \& Practices, vol. 3, no. 1, pp. 47-61, 2020. 